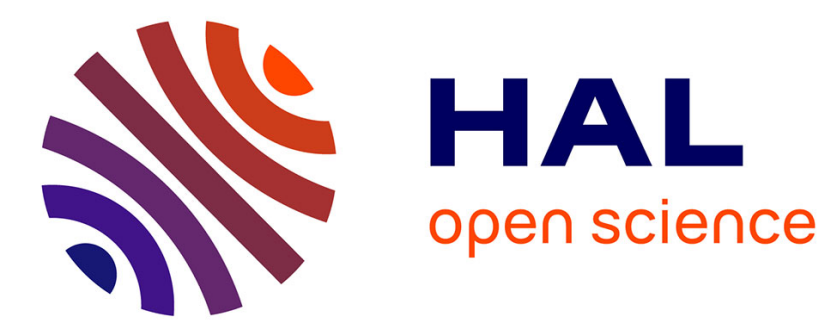

\title{
Transport Phenomena in Viscoelastic Fluids
}

Teodor Burghelea

\section{To cite this version:}

Teodor Burghelea. Transport Phenomena in Viscoelastic Fluids. Transport Phenomena in Complex Fluids, pp.83-166, 2019, 10.1007/978-3-030-35558-6_4 . hal-02868883

\section{HAL Id: hal-02868883 \\ https://hal.science/hal-02868883}

Submitted on 25 Nov 2020

HAL is a multi-disciplinary open access archive for the deposit and dissemination of scientific research documents, whether they are published or not. The documents may come from teaching and research institutions in France or abroad, or from public or private research centers.
L'archive ouverte pluridisciplinaire HAL, est destinée au dépôt et à la diffusion de documents scientifiques de niveau recherche, publiés ou non, émanant des établissements d'enseignement et de recherche français ou étrangers, des laboratoires publics ou privés. 


\title{
Transport phenomena in viscoelastic fluids
}

\author{
Teodor Burghelea \\ Université de Nantes, Nantes Atlantique Universités,Laboratoire de \\ Thermique et Energie de Nantes, UMR 6607, La Chantrerie, Rue \\ Christian Pauc, B.P. 50609, F-44306 Nantes Cedex 3, France
}

Abstract the abstract goes here

\section{Contents}

Contents

List of Figures

1 Molecular origins of viscoelasticity in dilute polymer solutions

2 Macroscopic flow phenomena triggered by the microscopic coil-stretch transition $\quad 16$

2.1 The rod climbing effect. . . . . . . . . . . . . . 16

2.2 Extrudate swell effect . . . . . . . . . . . . . . . 17

2.3 Drag reduction . . . . . . . . . . . . . . . 19

3 Hydrodynamic stability of dilute polymer solutions 20

4 Elastic turbulence in dilute polymer solutions: turbulence $\begin{array}{ll}\text { without inertia } & 23\end{array}$

4.1 Methods . . . . . . . . . . . . . . . . . . . . 24

4.2 Flow resistance . . . . . . . . . . . . . . . 28

4.3 Flow structure in a regime of Elastic Turbulence . . . . . . . 34

4.4 Lagrangian frame dynamics in a regime of Elastic Tuerbulence ...................... . . 55

Lagrangian frame flow intermittency . . . . . . . . . . . . 59

4.5 Applicability of the Taylor hypothesis in a regime of Elastic Turbulence ................. 63 
6 Characterisation of Elastic Turbulence in microscopic curvilinear flows

6.1 Onset and development of Elastic Turbulence in micro-channel, flow structure . . . . . . . . . . . . . . . . . 79

6.2 On the nature of the bifurcation towards Elastic Turbulence . 84

6.3 Statistics and spatial distribution of the velocity gradients; analysis of boundary layers . . . . . . . . . . 85

7 Efficient mixing by Elastic Turbulence $\quad 90$

7.1 The polymer solutions. . . . . . . . . . . . . . . 9 91

7.2 Passive scalar field measurements . . . . . . . . . . . . . . 93

7.3 Decay regime of mixing in a random micro flow . . . . . . . 93

7.4 Scaling of the mixing length with $\mathrm{Pe} \ldots \ldots \ldots 9 . \ldots 9$

7.5 Analysis of the mixing boundary layer . . . . . . . . . . 103

7.6 Spatial and temporal correlations of the passive scalar fluctuations . . . . . . . . . . . . . . . . . 104

8 Macroscopic heat transport by Elastic Turbulence 106

8.1 Experimental setup and methods . . . . . . . . . . . . . 107

8.2 Observation and characterisation of elastic turbulent flow states112

8.3 Heat transport by Elastic Turbulence . . . . . . . . . . . . . 114

8.4 Statistical and scaling properties of the temperature fluctuations: passive or active scalar? . . . . . . . . . . . . . 120

Bibliography

\section{List of Figures}

1 Posible configurations of a linear polymer molecule in a flow: (a) Coiled state (b) Stretched state. . . . . . . . . . . . . . . 15

2 (a) Sketch of a $2^{\text {nd }}$ order coil-stretch transition in a two dimensional flow when the vorticity $\omega$ increases while $A$ is large but constant. The width of the transition zone is $Z^{-1}$ (where $\mathrm{Z}$ is the number of monomers on one chain). (b) Sketch of a $1^{\text {st }}$ order coil-stretch transition in an extensional flow (c) Phase diagram corresponding to the coil-stretch transition: blue line second order, red line - first order. The sketches in each panel are reproductions of the sketches presented in Ref. De Gennes [1974] . . . . . . . . . . . . . . . . . 16 
3 Dependence of the steady fractional extension of Escherichia coli DNA molecules in planar extensional flow for increasing/decreasing values of the Deborah number, $D e=\lambda \dot{\epsilon}$. The plot is reproduced from Fig. 3(E) of Ref. Schroeder et al. [2003] with permission. we need to ask for the permission and pay for it 27 euros ! . . . . 17

4 (a) Schematic illustration of the Weissenberg effect. (b) Cross sectional view illustrating the physical mechanism of the Weissenberg effect. . . . . . . . . . . . . . . 18

5 (a) Newtonian fluid exiting a capillary tube, $D_{N}<D$. (b) Polymeric fluid exiting a capillary tube, $D_{P}>D$. . . . . . . . 18

6 (a) Sketch of a turbulent pipe flow with no polymer added. (b) Sketch of a turbulent pipe flow after a small amount of polymer has been added. . . . . . . . . . . . . . . . 19

7 Sketch of hydrodynamic stability diagram: $\mathbf{T}$-inertial turbulence, ET-elastic turbulence, DR-drag reduction, PEFI-purely elastic flow instabilities.

8 Overview of the swirling flow apparatus: C- fluid container, Drotating disk ,AR-1000-rheometer, CO-cylindrical optics, Llaser, M- plane mirror, CCD-digital camera, $\mathbf{P}$-LDV probe,BSAburst spectrum analyser, PMT-photomultiplier. . . . . . . . . . 25

9 Polymer relaxation obtained from oscillation measurements in a Mooney-Ewart geometry mounted on the AR-1000 rheometer. The full line is a fit $\dot{\gamma}^{-0.29}$. . . . . . . . . . . . . . 26

10 (A)Dependence of the mean power, $P$, on the control parameter, $W i$ : squares-increasing $W i$, circles-decreasing $W i$. The full line is a guide for the eye, $W i^{3.34}$. The dotted line is $W i^{2.85}$.The inset shows a power law fit, $W i^{3.34 \pm 0.05}$ (B) Dependence of the rms of power fluctuations, $P^{r m s}$, on $W i$ : squares-increasing $W i$, circles-decreasing $W i$. The full lines are power laws, $W i^{3.21}$. The inset shows the power law fits, $W i^{3.21 \pm 0.3}$. Data were collected in setup 2 in $\Omega$ forcing mode. . . . . . . . . . . . . . . .

11 Dependence of the onset of the primary elastic instability on geometric aspect ratio:(A) Shear Rate (B) Angular velocity. The symbols are: squares- $R_{d}=4.7 \mathrm{~cm}$, circles- $R_{d}=2.2 \mathrm{~cm}$, triangles$R_{d}=1.7 \mathrm{~cm}$. The full line is $\epsilon^{-1} \ldots \ldots \ldots \ldots$

12 (A) Time series (partially shown) of the injected power in constant $\Omega$ forcing regime for different $W i$. (B)pdf's of the power fluctuations in constant $\Omega$ forcing regime for different $W i$. The colors are: orange- $W i=5$, yellow- $W i=19$, red- $W i=24$, green$W i=31.5$, blue- $W i=40$. Data were collected in setup 2 . . . . . 
13 (A) Time series (partially shown) of the injected power in constant $T$ forcing regime for different $W i$. (B)pdf's of the power fluctuations in constant $T$ forcing regime for different $W i$. The colors are: blue- $W i=5$, green- $W i=17$, red- $W i=25$, yellow$W i=34$, black- $W i=46$. Data were collected in setup 2. . . . . 33

14 (A) Profiles of the average tangential velocity components, $V_{\theta}$, for several values of $W i$. The inset shows a typical laminar profile of the tangential velocity component. (B)Profiles of the average radial velocity components, $V_{r}$, for several values of $W i$. The colors are: black- $W i=2.48$, green- $W i=4.41$, red- $W i=11.1$, orange- $W i=15$, blue- $W i=18$.Data were collected in setup 1 at middle distance between plates. . . . . . . . . . . . . 34

15 Instantaneous (left column) and time averaged (right column)velocity fields for different values $W i$ : a) $W i=2.48 \mathbf{b}$ ) $W i=9.88$ (c) $\mathrm{Wi}=18.96$. Data were collected in setup 1 at middle distance between plates.

16 Distribution of the radial velocity component(left column) and of the tangential velocity components ( right column) for different values of $W i$ :(a) $W i=2.48$ (b) $W i=9.88$ (c) $W i=18.96$. Data were collected in setup 1 at middle distance between plates. . . . 37

17 Fields of fluctuations of the tangential velocity component at different $W i$ :(a) $W i=8.32$.(b) $W i=9.88$.(c) $W i=11.1$.(d) $W i=12.72$.(e) $W i=13.83$.(f) $W i=17$ (g) $W i=19$. Data were collected in the setup 1 at middle distance between plates. . 38

18 Turbulence intensity versus $W i$ at different radial positions: squares$r=1 \mathrm{~cm}$, up triangles- $r=0.7 \mathrm{~cm}$, down triangles $-r=1.5 \mathrm{~cm}$, rhumbs- $r=1.74 \mathrm{~cm}$, left triangles- $r=1.89 \mathrm{~cm}$. The arrow indicates the onset of the elastic instability. The full line is a guide for the eye, $W i^{0.5}$. The data were collected in setup 1. . . . . . . 39

19 Turbulence intensity versus the reduced radial coordinate at different $W i$ : rhumbs- $W i=31.56$, squares- $W i=27.73$, circles$W i=23.67$, up triangles- $W i=19.29$, down triangles- $W i=$ 15.48 ,stars- $W i=7.54$, half filled triangles- $W i=2.82$. The inset shows the dependence of the turbulence intensity on $W i$ at $r / R_{c}=0.85$ obtained from LDV measurements. The full line is a power law fit, $I_{t} \propto W i^{0.49 \pm 0.06}$. The arrow indicates the onset of the elastic instability. The data were collected in setup 2. . . 40

20 Eulerian correlation times of the tangential velocity component as function of $W i$ : squares-setup 1 , circles-setup 2. . . . . . . . . 42 
21 Power spectra of the fluctuations of tangential velocity component at different $W i$ : circles- $W i=4.41$, up triangles- $W i=$ 5.72, down triangles- $W i=8.32$, left triangles $W i=11.1$, right triangles- $W i=12.7$, hexagons- $W i=13.8$, diamonds- $W i=16$, half filled squares-Wi $W 18$, empty circles- $W i=19$. Data were collected in setup $1 . \ldots \ldots \ldots \ldots \ldots \ldots$

22 Average gradient of the tangential velocity component in the radial direction, $\frac{\partial v_{\theta}}{\partial r}$, at different $W i$ : (a) $W i=8.32$.(b) $W i=$ 9.88.(c) $W i=11.1$. (d) $W i=12.72$. (e) $W i=13.83$.(f) $W i=17(\mathrm{~g}) W i=19$. Data were collected in setup 1 at middle distance between plates. The squared pattern slightly visible in panel (a) is a result of combined peak locking effect and numerical differentiation and should be disregarded. . . . . . . . . . . .

23 Average gradient of the radial velocity component in the radial direction, $\frac{\partial v_{r}}{\partial r}$, at different $W i$ : (a) $W i=8.32$.(b) $W i=$ 9.88.(c) $W i=11.1$.(d) $W i=12.72$.(e) $W i=13.83$.(f) $W i=$ 17 (g) $W i=19$. Data were collected in setup 1 at middle distance between plates. . . . . . . . . . . . . . .

24 Average vertical component of the vorticity, $\omega_{r \theta}$, at different $W i$ : (a) $W i=8.32$.(b) $W i=9.88$.(c) $W i=11.1$.(d) $W i=$ 12.72.(e) $W i=13.83$.(f) $W i=17(\mathrm{~g}) W i=19$. Data were collected in setup 1 at middle distance between plates. The squared pattern visible in panels (a), (b) is a result of combined peak locking effect and numerical differentiation and should be disregarded. . . . . . . . . . . . . . . . . .

25 (A) Profiles of the average radial gradient of the tangential velocity at different $W i$ : black- $W i=19$, red- $W i=18$, green- $W i=$ 11 , blue- $W i=16$, magenta- $W i=13.83$, olive- $W i=11.1$, dark blue- $W i=9.88$, yellow- $W i=8.32$, orange- $W i=5.73$, pink$W i=2.48$. (B) Dependence of the average radial gradient of the tangential velocity on $W i$ at different radial positions: squares$r / R_{c}=0.3$, circles $-r / R_{c}=0.66$, up triangles $-r / R_{c}=0.4$, down triangles- $r / R_{c}=0.2$, left triangles- $r / R_{c}=0.1$, right triangles$r / R_{c}=0.7$, stars- $r / R_{c}=0.5$. The arrow indicates the onset of the elastic instability. Data were collected in setup 1 at middle distance between plates. 
26 (A) Profiles of the rms of fluctuations of the radial gradient of the tangential velocity at different $W i$ : black-Wi $=19$, red$W i=18$, green- $W i=11$, blue- $W i=16$, magenta- $W i=13.83$, olive- $W i=11.1$, dark blue- $W i=9.88$, yellow- $W i=8.32$, orange$W i=5.73$, pink-Wi $=2.48$. (B) Dependence of the rms of fluctuations of the radial gradient of the tangential velocity on $W i$ at different radial positions: squares- $r / R_{c}=0.3$, circles$r / R_{c}=0.66$, up triangles- $r / R_{c}=0.4$, down triangles- $r / R_{c}=$ 0.2 , left triangles- $r / R_{c}=0.1$, right triangles- $r / R_{c}=0.7$, stars$r / R_{c}=0.5$. The arrow indicates the onset of the elastic instability. Data were collected in setup 1 at middle distance between plates. . . . . . . . . . . . . . . . . .

27 (A) Profiles of the average vorticity, $\left\langle\omega_{r \theta}\right\rangle$, at different $W i$ : black- $W i=19$, red- $W i=18$, green- $W i=11$, blue- $W i=16$, magenta- $W i=13.83$, olive- $W i=11.1$, dark blue- $W i=9.88$, yellow- $W i=8.32$, orange- $W i=5.73$, pink- $W i=2.48$. (B) Dependence of the average vorticity on $W i$ at different radial positions: squares- $r / R_{c}=0.3$, circles- $r / R_{c}=0.66$, up triangles$r / R_{c}=0.4$, down triangles- $r / R_{c}=0.2$, left triangles- $r / R_{c}=$ 0.1 , right triangles- $r / R_{c}=0.7$, stars- $r / R_{c}=0.5$. The arrow indicates the onset of the elastic instability. Data were collected in setup $1 \ldots \ldots \ldots \ldots \ldots \ldots \ldots \ldots$

28 (A) Profiles of the rms of fluctuations of the vorticity, $\omega_{r \theta}^{r m s}$, at different $W i$ : black- $W i=19$, red- $W i=18$, green- $W i=11$, blue- $W i=16$, magenta- $W i=13.83$, olive- $W i=11.1$, dark blue- $W i=9.88$, yellow- $W i=8.32$, orange- $W i=5.73$, pink$W i=2.48$. (B) Dependence of the rms of fluctuations of the vorticity on $W i$ at different radial positions: squares- $r / R_{c}=0.3$, circles- $r / R_{c}=0.66$, up triangles- $r / R_{c}=0.4$, down triangles$r / R_{c}=0.2$, left triangles- $r / R_{c}=0.1$, right triangles- $r / R_{c}=0.7$, stars- $r / R_{c}=0.5$. The arrow indicates the onset of the elastic instability. Data were collected in setup 1. . . . . . . . . . 50 
29 (A) Profiles of the average radial gradient of the tangential velocity at different $W i$ :black- $W i=34.5$, red- $W i=32.3$, green$W i=27.73$, blue- $W i=26.13$, magenta- $W i=19.29$, dark yellow- $W i=17.43$, dark blue- $W i=14.48$, yellow- $W i=10$, orange- $W i=5.4$, pink- $W i=2.82$. (B) Dependence of the average radial gradient of the tangential velocity on $W i$ at different radial positions: squares- $r / R_{c}=0.33$, circles- $r / R_{c}=0.66$, up triangles- $r / R_{c}=0.4$, down triangles- $r / R_{c}=0.2$, left triangles$r / R_{c}=0.1$, right triangles- $r / R_{c}=0.7$, stars- $r / R_{c}=0.5$, half filled squares- $r / R_{c}=0.8$, half filled circles- $r / R_{c}=0.9$. The arrow indicates the onset of the elastic instability. Data were collected in setup $2 . \ldots \ldots \ldots \ldots \ldots \ldots$

30 (A) Profiles of the rms of fluctuations of the radial gradient of the tangential velocity at different $W i$ : black- $W i=34.5$, red$W i=32.3$, green- $W i=27.73$, blue- $W i=26.13$, magenta- $W i=$ 19.29 , dark yellow- $W i=17.43$, dark blue- $W i=14.48$, yellow$W i=10$, orange- $W i=5.4$, pink- $W i=2.82$.(B) Dependence of the rms of fluctuations of the radial gradient of the tangential velocity on $W i$ at different radial positions: squares $r / R_{c}=0.33$, circles- $r / R_{c}=0.66$, up triangles- $r / R_{c}=0.4$, down triangles$r / R_{c}=0.2$, left triangles- $r / R_{c}=0.1$, right triangles- $r / R_{c}=$ 0.7 , stars- $r / R_{c}=0.5$, half filled squares- $r / R_{c}=0.8$, half filled circles- $r / R_{c}=0.9$. The arrow indicates the onset of the elastic instability. Data were collected in setup $2 \ldots \ldots \ldots \ldots \ldots$

31 (A)Profiles of the average vorticity, $\left\langle\omega_{r, \theta}\right\rangle$, at different $W i$ : black$W i=34.5$, red- $W i=32.3$, green- $W i=27.73$, blue- $W i=$ 26.13 , magenta- $W i=19.29$, dark yellow- $W i=17.43$, dark blue$W i=14.48$, yellow- $W i=10$, orange- $W i=5.4$, pink- $W i=2.82$ (B) Dependence of the average vorticity on $W i$ at different radial positions: squares- $r / R_{c}=0.33$, circles $-r / R_{c}=0.66$, up triangles- $r / R_{c}=0.4$, down triangles- $r / R_{c}=0.2$, left triangles$r / R_{c}=0.1$, right triangles- $r / R_{c}=0.7$, stars- $r / R_{c}=0.5$, half filled squares- $r / R_{c}=0.8$, half filled circles- $r / R_{c}=0.9$. The arrow indicates the onset of the elastic instability. Data were collected in setup $2 . \ldots \ldots \ldots \ldots \ldots \ldots \ldots$ 
32 (A) Profiles of the rms of fluctuations of the vorticity, $\omega_{r, \theta}^{r m s}$, at different $W i$ : black- $W i=34.5$, red- $W i=32.3$, green- $W i=$ 27.73 , blue- $W i=26.13$, magenta- $W i=19.29$, dark yellow- $W i=$ 17.43 , dark blue- $W i=14.48$, yellow- $W i=10$, orange- $W i=5.4$, pink- $W i=2.82$.(B) Dependence of the rms of fluctuations of the vorticity on $W i$ at different radial positions: squares- $r / R_{c}=0.33$, circles- $r / R_{c}=0.66$, up triangles- $r / R_{c}=0.4$, down triangles$r / R_{c}=0.2$, left triangles- $r / R_{c}=0.1$, right triangles- $r / R_{c}=$ 0.7 , stars- $r / R_{c}=0.5$, half filled squares- $r / R_{c}=0.8$, half filled circles- $r / R_{c}=0.9$. The arrow indicates the onset of the elastic instability. Data were collected in setup $2 \ldots$. . . . . . . . . . 54

33 Numerical tracers at $t=0$ (a) and $t=1 \mathrm{~s}$ (b) for $W i=33$. The false color maps the radial coordinate. . . . . . . . . . . 58

34 Cramer functions of particle pair separation rates for 25 different separation times (ranging from 0.16 to $2.08 \mathrm{~s}$ ) at $W i=34$. The full line is the parabolic fit. . . . . . . . . . . . . .

35 Finite time Lyapunov exponents defined by the location of the minima of the Cramer rate functions of the particle pairs separations versus Wi: squares, from PTV approach, circles from PIV based approach. The arrow indicates the onset of the primary elastic instability. The inset shows the dependence of FTLE on the separation time; the horizontal axis is normalised by the period of rotation of the disk. . . . . . . . . . . . . . 60

36 Generalised Lyapunov exponents as a function of the order $q$ of the moments at different $W i$ : squares- $W i=33$, triangles$W i=2.82$. The full line is the parabolic fit, which gives $\mu=$

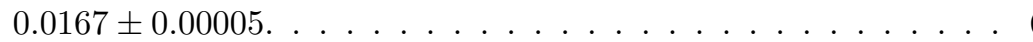

37 Normalized (see text) high order moments at different $W i$ : squares, - $W i=1.9$, up triangles- $W i=4.8$, diamonds $-W i=29$, circles$W i=33.3$, crosses- $W i=38$, right triangles- $W i=56$, left triangles- $W i=58$. The inset shows an estimate of the degree of the flow intermittency versus $W i$. . . . . . . . . . . . 62

38 Schematic view of the flow geometry: (M)- mean flow line, (L) measured Lagrangian trajectory (C) - circular arc. . . . . . . . 64

39 Simultaneous tangential velocity time series (partially shown) at $W i=57.6$ for three values of the displacement along the mean flow line: (a) $s=0 \mathrm{~cm}$, (b) $s=1.5 \mathrm{~cm}$ (c) $\mathrm{s}=3.2 \mathrm{~cm} . \ldots \ldots 6$

40 Space -time correlation surface. . . . . . . . . . . 67

41 Scaled cross correlation functions for displacements s along the mean flow line ranging from $7 \mathrm{~mm}$ to $4 \mathrm{~cm}$. The dotted line is a parabolic fit. . . . . . . . . . . . . . . 68 
42 Space dependence of cross correlation functions at fixed time delays: squares- $\tau=0$, circles- $\tau=\tau_{\max }$. The full lines are parabolic fits. . . . . . . . . . . . . . . . . 69

43 The cross correlation of velocity fluctuations, $C_{r r}(\tau, s)$ vs. the delay time $\tau$ for different values of the displacement $\mathrm{s}$ along the mean flow line. . . . . . . . . . . . . . . . . . .

44 Bottom-left axis: the delay time $\tau_{\max }$ that maximises the cross correlation functions vs. the length of the displacement along three different paths: squares-circular arcs, circles-mean flow line, triangles- Lagrangian trajectory. The horizontal axis is normalised by the mean velocity at $\mathrm{s}=0$. The full lines are linear fits with slopes $0.6,0.7$ and 0.73 respectively. The dotted line has slope 1. Top-right axis: the delay time $\tau_{e}$ (see text) vs. the transit time $\tau_{t}$. The full line is a linear fit with slope 0.96. The initial position was $\vec{r}=\left(\frac{2 R_{c}}{3}, 0\right) . \ldots \ldots \ldots \ldots$

45 Validity of the TH (see text for discussion) vs. the radial coordinate. . . . . . . . . . . . . . . . . 71

46 Second order velocity structure functions: squares - resulted from direct measurements, circles derived by using the Taylor hypothesis. Here we have considered displacements along the mean flow line and the initial position was: (a) $\vec{r}=\left(R_{c} / 3,0\right)$ (b) $\vec{r}=\left(2 \cdot R_{c} / 3,0\right) \ldots \ldots \ldots \ldots \ldots \ldots \ldots$

47 (a) Schematic view of the serpentine micro-channel. (b) Schematic view of the experimental setup (not in scale): MCC - microchannel chip, I - micro-channel inlet, O - micro-channel outlet, AT - adhesive tape, MO - microscope objective, DM - dichroic mirror, LP - led, EXF - excitation filter, EMF - emission filter, CCD - digital camera. . . . . . . . . . . . . . . . 79

48 Epifluorescent flow micrographs acquired at (a) $\Delta p=63 \mathrm{~Pa}$ (Wi $=0.36$ - laminar regime) (b) $\Delta p=2.3 k P a(W i=18$ elastic turbulent regime). A X10 magnification objective was used. . . . . . . . . . . . . . . . . . . . 80

49 Dependence of the time averaged tangential velocity component on the driving pressure (bottom horizontal axis) and and on the integral Weissenberg number (top horizontal axis). The full/empty symbols refer to the increasing/decreasing branches of the pressure ramp. The shaded regions highlight the level of fluctuations. The full line is a linear fit. . . . . . . . . . . . 80

50 Velocity Profiles . . . . . . . . . . . . . . . . . 81

51 Time Series . . . . . . . . . . . . . . . . . 82 
52 Top: Time series of the tangential velocity component acquired at $W i=9.5$. The bottom plots illustrate maps of the velocity modulus at several time instants indicated on the top. The full lines in each bottom panel are streamlines. . . . . . . . . 83

53 Rms Of Fluctuations . . . . . . . . . . . . . . . . . 84

54 Spatial distribution of the time averaged second invariant of the rate of strain measured at different Weissenberg numbers indicated in the top inserts. The units of the colorbars are $s^{-1}$. . . 86

55 Profiles of the gradients . . . . . . . . . . . . . 87

56 Dependence of the reduced width of the inner boundary layer $(\square, \boldsymbol{\square})$ and of the outer boundary layer $(\bullet, \circ)$ on the global Weissenberg number. The full/empty symbols refer to increasing/decreasing global Weissenberg numbers, respectively. . . . . . . . . . . 88

57 Velocity Gradients . . . . . . . . . . . . . . . . . 89

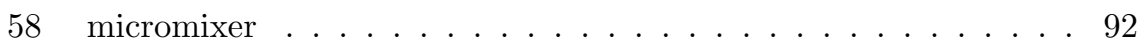

59 Confocal scanning system: PMT-photo-multiplier, PH-pin hole, L1-focusing lens ,FF-fluorescence filter, DM-dichroic mirror, EF-excitation light selection filter, L-laser, Y-SM-Y direction scanning mirror, X-SM-X direction scanning mirror, L2-objective lens, M-micro-channel. . . . . . . . . . . . . . . . . 94

60 Middle plane horizontal confocal snapshot. The flow is seeded with $0.2 \mu \mathrm{m}$ fluorescent spheres. The driving pressure is $120 \mathrm{~Pa}$ per channel segment . . . . . . . . . . . . . . . 94

61 (A)Epifluorescent microphotograph of the entrance area of a micro-channel. Wide triangular region in front of a curvilinear channel allows to adjust equal flow rates for polymer solutions with (from the top) and without FITCD. (B)Confocal micrograph of the flow in the micro-channel (at $\mathrm{N}=30$ ) without polymers added. Left wall of the channel is shown by a dotted line. Confocal images of mixing in a random micro flow of a dilute polymer solution at different locations downstream: $(\mathrm{C}) \mathrm{N}=5(\mathrm{D}) \mathrm{N}=11(\mathrm{E}) \mathrm{N}=17 \ldots \ldots \ldots \ldots$

62 Distribution of tracer concentration in the vertical cross-section of the micro-channel at different locations downstream: $(\mathrm{A}) \mathrm{N}=5$ $(\mathrm{B}) \mathrm{N}=7(\mathrm{C}) \mathrm{N}=9(\mathrm{D}) \mathrm{N}=11(\mathrm{E}) \mathrm{N}=13(\mathrm{~F}) \mathrm{N}=15(\mathrm{G}) \mathrm{N}=17(\mathrm{H}) \mathrm{N}=25.99$

63 Time average of FITCD concentration, $\bar{c}$, as a function of the normalized coordinate across the micro-channel at different locations downstream: red- $N=7$, green- $N=11$ and blue-N=41. .100 
64 Space-time plots of FITCD distribution across the channel at different locations downstream: (A)N=5 (B) $\mathrm{N}=13(\mathrm{C}) \mathrm{N}=21$ (D) $\mathrm{N}=35$. Confocal scanning was done along the same line across the channel in the midplane at equal distances from the half-ring interconnections, with even time intervals of 0.0177 s. . . . . . . . . 101

65 The first (squares) and second(circles) order moments decay vs. position, $\mathrm{N}$, at $P e=4.15 \times 10^{5}$; solid lines are the fit. Inset: the decay exponents of the moments, $\gamma_{i}$, as a function of the moment order, i, at different Pe: diamonds, $P e=1.6 \times 10^{4}$; up triangles, $P e=3.3 \times 10^{4}$; squares, $P e=9.09 \times 10^{4}$; circles, $P e=1.74 \times 10^{5} ;$ down triangles, $P e=4.15 \times 10^{5}$. . . . . . 102

66 The mixing length, measured in the number of turns, $N_{m i x}$, vs. $P e$ : squares are the data, the solid line is the fit, and the dotted line shows the logarithmic dependence. . . . . . . . . . . . . . . 103

67 Spatial dependence of the first (blue line) and second (black line) order moments of FITCD concentration fluctuations across microchannel. Inset: width of the boundary layer, $W$, vs. Pe. The solid line is the fit. . . . . . . . . . . . . . . . . . . . . . 104

68 (A) Correlation coefficients for the tracer concentration vs. $\Delta x / d$. (B) Correlation coefficients for the tracer concentration vs. $t$ in the bulk of the microdcopic flow. (C) Time auto correlation functions for the tracer concentration vs. near the boundary of the microscopic flow. The colours are: black- $P e=4.15 \times 10^{5}$, red- $P e=1.74 \times 10^{5}$, blue- $P e=9.09 \times 10^{4}$, green- $P e=3.37 \times 10^{4}$, orange- $P e=1.68 \times 10^{4} \ldots \ldots \ldots \ldots \ldots \ldots$

69 Schematic view of the experimental setup: $\mathbf{R}$ - the shaft of the rheometer, D - rotating disk, FC - fluid container, CFB - circulating fluid bath, $\mathbf{T}_{\mathbf{1}-\mathbf{6}}$ - thermocouples, $\mathbf{R B}$ - reference box, $\mathbf{A}_{\mathbf{2}} \mathbf{D}$ - analogue to digital converter, $\mathbf{L}$ - solid state laser, LS laser sheet, $\mathbf{C O B}$ - cylindrical optics block, $\mathbf{M}$ - planar mirror, CCD - video camera, PC - personal computer. . . . . . . . 108 
70 (a) Flow curves measured during controlled rate flow ramps. The insert presents the temperature dependence of the viscosity measured at $\dot{\gamma}=1 s^{-1}$ and the full line is an Arrhenius fit. (b) Stress relaxation data. The full lines are exponentially decaying functions characterising the slow relaxation mode. The dash-dot line is a guide for the eye, $\sigma / \sigma_{0} \propto e^{-t / 2.6( \pm 0.2)}$, highlighting the fast decay mode. The temperature dependence of the longest relaxation time is presented in the insert. The error bars are defined via the nonlinear fitting error. In each panel, the symbols refer to different operating temperatures: squares $(\square)-T=17^{\circ} C$, circles $(\circ)-T=19^{\circ} C$, triangles $(\triangle)-T=21^{\circ} C$. . . . . . . 109

71 (a) Dependence of the normalised time averaged injected power $\bar{P} / \bar{P}_{\text {lam }}$ on the Weissenberg number $W i$. The full line is a guide for the eye, $\bar{P} / \bar{P}_{l a m} \propto W i^{0.67}$. (b) Dependence of the reduced rms of the power fluctuations $P^{r m s} / P_{l a m}^{r m s}$ on the Weissenberg number $W i$. The full line is a guide for the eye, $P^{r m s} / P_{\text {lam }}^{r m s} \propto$ $W i^{3}$. The full/empty symbols refer to increasing/decreasing $W i$. The vertical arrow indicates the onset of the primary elastic instability $W i_{c} \ldots \ldots \ldots \ldots 13$

72 Top row: time averaged flow patterns for various Weissenberg numbers indicated in the top inserts. The colour map refers to the modulus of the in-plane velocity. For the clarity of the presentation, the overlapped vector fields were down-sampled by a factor of 2 along each direction. Bottom row: time averaged vorticity, $\left\langle\omega_{z}\right\rangle_{t}$. The measurements were performed in isothermal conditions at the room temperature $T_{0} \ldots \ldots$. . . . . . . . 114 
73 (a) Time series of the reduced temperature measured with the sucrose solvent at $R e \approx 125$ : circle $(\circ)-T_{1}$, square $(\square)-T_{2}$, up triangle $(\triangle)-T_{3}$, down triangle $(\nabla)-T_{4}$, left triangle $(\triangleleft)$ $T_{5}$, hexagon $(\square)-T_{6}$. All measurements were performed at a distance $r=R_{c} / 2$ from the symmetry axis of the flow container FC. The full lines are nonlinear fitting functions (see explanation in the text). The dependence of the fit parameter $B$ (see text for explanation) on the vertical coordinate is presented in the insert.(b) Time series of the reduced temperature measured at three Weissenberg numbers indicated in the inserts of each panel. The symbols in each panel refer to the vertical position of the thermocouple (see Fig. 69): circle (o) $-T_{1}$, square $(\square)-T_{2}$, up triangle $(\triangle)-T_{3}$, down triangle $(\nabla)-T_{4}$, left triangle $(\triangleleft)$ $T_{5}$, hexagon $(\square)-T_{6}$. All measurements were performed at a distance $r=R_{c} / 2$ from the symmetry axis of the flow container FC.. . . . . . . . . . . . . . . . . 115

74 Dependence of the efficiency factor $b$ obtained at various vertical positions indicated in the insert on the Weissenberg number. The symbols refer to the position along the vertical axis $z$ (see Fig. 69): ( $\square)-T_{1},(\circ)-T_{2},(\triangle)-T_{3},(\nabla)-T_{4},(\diamond)-T_{5},(\triangleleft)-T_{6}$. The vertical dashed line indicates the onset of the primary elastic instability $W i_{c} \ldots \ldots \ldots \ldots \ldots$. . . . . . . . . . 118

75 Dependence of the intensity factor $b$ on the vertical coordinate. The symbols refer to the Weissenberg number: rhombs $(\diamond)$ $W i=0$, squares $(\square)-W i=0.8$, triangles $(\triangle)-W i=7.7$, circle

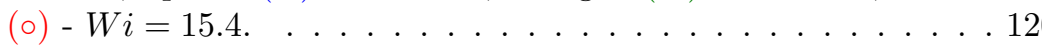

76 Time series of the fluctuating part of the reduced temperature $\theta_{r}$ obtained from the measurements of the thermocouple $T_{4}$ (see Fig. 69 ) at various Weissenberg numbers: (a) $W i=0$ (b) $W i=0.8$, (c) $W i=7.7$, (d) $W i=15.4 . \ldots \ldots . \ldots 12$

77 Space distribution of the rms of the fluctuations of the reduced temperature, $\theta_{r}^{r m s}$ measured at various Weissenberg numbers: rhombs $(\diamond)-W i=0$, squares $(\square)$ - Wi $=0.8$, triangles $(\triangle)$ $W i=7.7$, circle $(\circ)-W i=15.4 \ldots \ldots \ldots \ldots$

78 Probability distribution functions and moments . . . . . . . . . . 124 
79 Pdf's of the fluctuations of the reduced temperature measured at various vertical positions and several $W i$ : (a) $W i=0$ (b) $W i=0.8$ (c) $W i=7.7$ (d) $W i=15.4$. In each panel the symbols refer to different thermocouples (see Fig. 69): squares $(\boldsymbol{\square})-T_{1}$, circles $(\bullet)-T_{2}$, up triangles $(\Delta)-T_{3}$, down triangles $(\boldsymbol{\nabla})-T_{4}$, rhombs $(\diamond)-T_{5}$, left triangles $(\triangleleft)-T_{6}$. The full lines in panels $(\mathrm{a}, \mathrm{b})$ are Gaussian fitting functions. . . . . . . . . . . 125

80 Autocorrelation and spectra . . . . . . . . . . 126

\section{Molecular origins of viscoelasticity in dilute polymer solutions}

A distinct class of complex fluids is represented by dilute solutions of high molecular weight linear polymers. The dilute limit refers to the situation when polymer-polymer interaction can be neglected. A typical linear polymer strand consists of a large number of sequentially bonded monomer units of molecular weights typically of the order of $10^{2} \mathrm{Da}$ with a total molecular weight $M$ of the order $10^{6} \div 10^{7} \mathrm{Da}$.

The simplest picture of a high molecular weight linear polymer molecule immersed in a low molecular weight (Newtonian) solvent is that of an elastic spring with two beads connected at its ends (elastic dumb-bell model). In the absence of flow and external forces acting on the chain, the most probable state of the molecule is the coiled state, Fig. 1(a). In the presence of flow, each bead interacts hydrodynamically with the solvent via Stokes like frictional forces (the Reynolds number based on the size of the polymer molecule is negligibly small). If the magnitude of the largest eigenvalue of the velocity gradient tensor becomes of the order of the inverse characteristic relaxation time of the polymer molecule, the coil-stretch transition occurs, Fig. 1(b).

The coil-stretch transition was first predicted by Pierre Gilles de Gennes in his seminal 1974 paper, De Gennes [1974]. As the microscopic coilstretch transition is naturally associated to the emergence of a polymer component in the stress tensor, its dynamics will consequently impact the macroscopic flow features discussed through this chapter. For this reason, a brief discussion of the main elements and predictions of the de Gennes's theory is in order. A first subtle element of the theory was to realise that the dynamics of the coil-stretch transition depends on the kinematics of the flow. Thus, two cases are considered: shear flows and shear free (extensional) flows. A two dimensional flow field $\left(V_{x}, V_{y}\right)$ is characterised by the vorticity $\omega=\frac{1}{2}\left(\frac{\partial V_{x}}{\partial y}-\frac{\partial V_{y}}{\partial x}\right)$ and the extensional part $A=\frac{1}{2}\left(\frac{\partial V_{x}}{\partial y}+\frac{\partial V_{y}}{\partial x}\right)$. The 


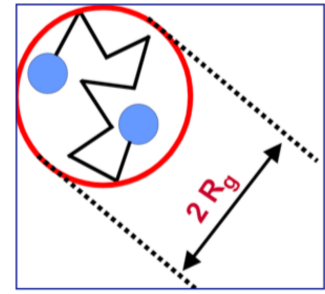

(a)

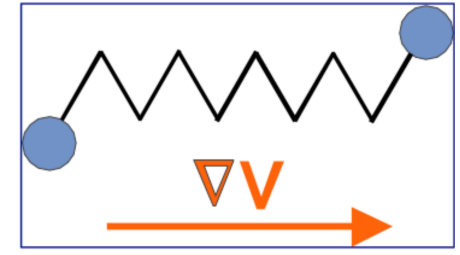

(b)

Figure 1. Posible configurations of a linear polymer molecule in a flow: (a) Coiled state (b) Stretched state.

sketch presented in Fig. 2(a) exemplifies the coil-stretch transition for the case when the vorticity $\omega$ is gradually decreased while $A$ is maintained constant (but large). The transition is a $2^{\text {nd }}$ order (imperfect) bifurcation and the width of the transitional region is approximately $Z^{-1}$ where $Z$ is the number of monomers in the chain, De Gennes [1974].

The nature of the bifurcation from a coiled to a stretched state changes when a shear free flow is considered: $V_{x}=\dot{\epsilon} x, V_{y}=-\dot{\epsilon} y$.

In this case the the dependence of the fractional extension of the polymer molecules folds back and in a certain range of rates of extension $\dot{\epsilon}$ and a bistable equilibrium is observed. There is one critical value of $\dot{\epsilon}=\dot{\epsilon}^{*}$ at which the system (if it is operated very slowly) will switch from a coiled state to a stretched one. A first order phase transition takes place at $\dot{\epsilon}=\dot{\epsilon}^{*}$.

A first experimental confirmation of the de Gennes theory has been reported by Schroeder and coworkers for the case of a planar extensional flow of a dilute solution of highly flexible Escherichia coli DNA molecules, Schroeder et al. [2003]. By tracking a statistically relevant number of individual polymer molecules stained with a fluorescent compound for various Deborah numbers $D e=\lambda \dot{\epsilon}$ they confirm the $1^{\text {st }}$ order bifurcation conjectured by de Gennes in 1974 for the case of a steady extensional flow, Fig. 3.

To our best knowledge, the prediction of a $2^{\text {nd }}$ order bifurcation for the case of a steady shear flow (see the sketch in Fig. 2(a)) still awaits an experimental validation. 

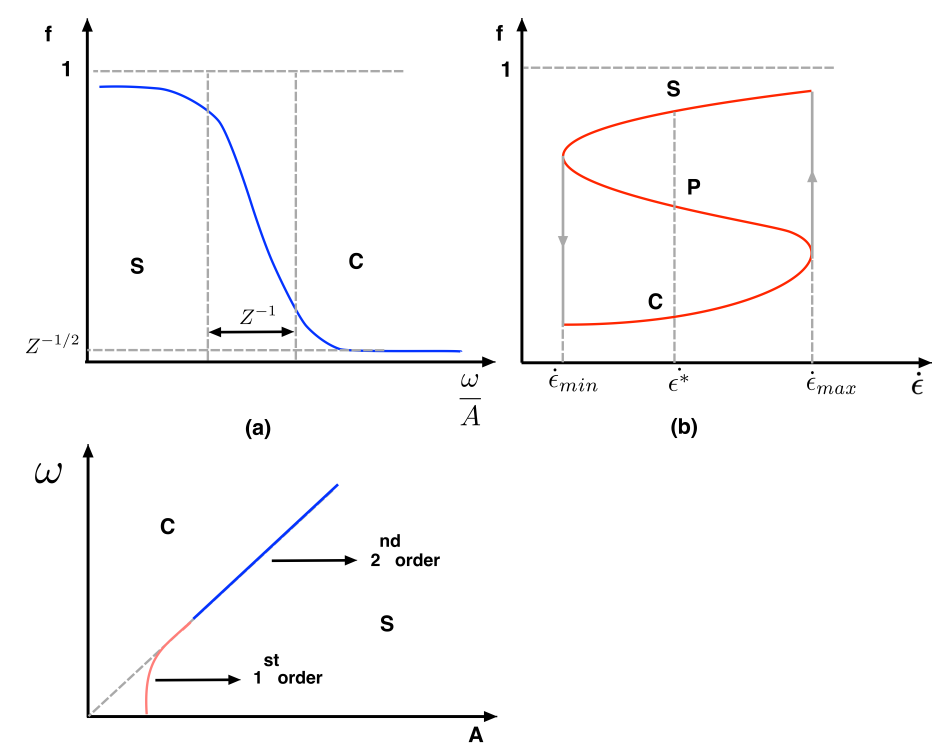

(b)

(c)

Figure 2. (a) Sketch of a $2^{\text {nd }}$ order coil-stretch transition in a two dimensional flow when the vorticity $\omega$ increases while $A$ is large but constant. The width of the transition zone is $Z^{-1}$ (where $\mathrm{Z}$ is the number of monomers on one chain). (b) Sketch of a $1^{\text {st }}$ order coil-stretch transition in an extensional flow (c) Phase diagram corresponding to the coil-stretch transition: blue line - second order, red line - first order. The sketches in each panel are reproductions of the sketches presented in Ref. De Gennes [1974].

\section{Macroscopic flow phenomena triggered by the microscopic coil-stretch transition}

\subsection{The rod climbing effect}

The generation of a macroscopic elastic stress component associated to the microscopic coil-stretch transition is responsible for a number of somewhat counterintuitive flow phenomena. The best known such effect is, probably, the rod-climbing or the Weissenberg effect, Weissenberg [1947].

As schematically illustrated in Fig. 4 (a), a polymeric fluid possessing "enough" elasticity climbs on a rod rotating at a constant angular speed $\Omega$ in the fluid rather being repelled by the centrifugal force (which is obviously what would happen if the fluid was Newtonian). 


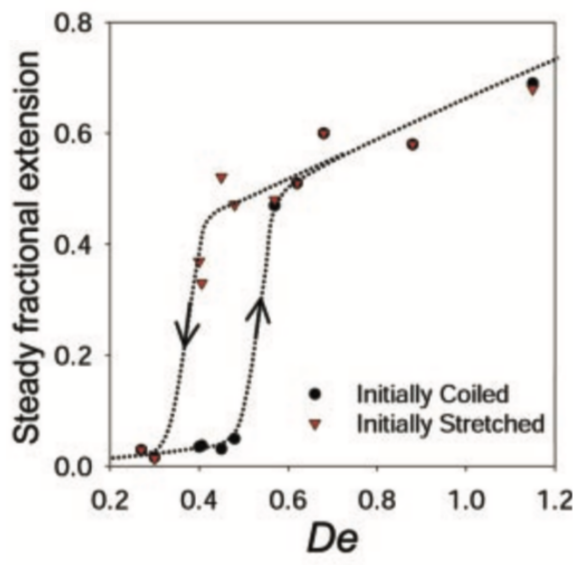

Figure 3. Dependence of the steady fractional extension of Escherichia coli DNA molecules in planar extensional flow for increasing/decreasing values of the Deborah number, $D e=\lambda \dot{\epsilon}$. The plot is reproduced from Fig. 3(E) of Ref. Schroeder et al. [2003] with permission. we need to ask for the permission and pay for it 27 euros! .

A simple phenomenological explanation of this effect may be formulated as follows. The individual polymer molecules get stretched along the stream lines which are closed circles which generates an extra tension along these lines which generates a $\mathrm{n}$ inwards fluid motion which makes the polymeric fluid climb the rod. This is schematically illustrated in Fig. 4 (b).

More rigorously, it can be shown that actually the first normal stress difference $N_{1}=\sigma_{\theta \theta}-\sigma_{r r}$ which is proportional to the square of the rate of shear but generally negative generates a body force $F=\frac{N_{1}}{r}$ called hoop stress oriented inwards, Bird et al. [1977]. In the case of a Newtonian $N_{1}=0$, the only acting force is the centrifugal one and the liquid is pushed outwards.

\section{$2.2 \quad$ Extrudate swell effect}

If a Newtonian fluid exits a capillary of diameter $D$ at low Reynolds number it forms a jet of a smaller diameter $D_{N}<D$ as schematically illustrated in Fig. 5(a). Typically $D_{N}$ is up to ten percents smaller than the diameter of the capillary. This phenomenon is called "vena contraction" and was first described by Torricelli in 1643, Torricelli [1644]. 


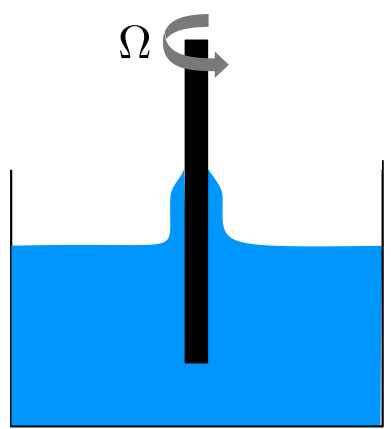

(a)

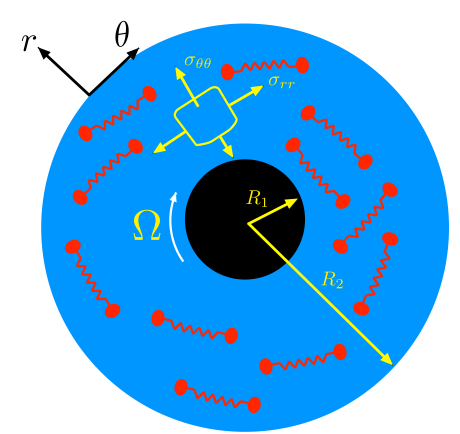

(b)

Figure 4. (a) Schematic illustration of the Weissenberg effect. (b) Cross sectional view illustrating the physical mechanism of the Weissenberg effect.

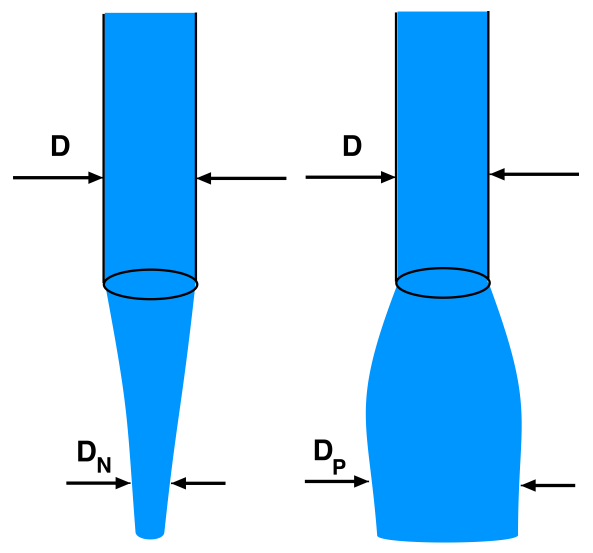

(a)

(b)

Figure 5. (a) Newtonian fluid exiting a capillary tube, $D_{N}<D$. (b) Polymeric fluid exiting a capillary tube, $D_{P}>D$. 


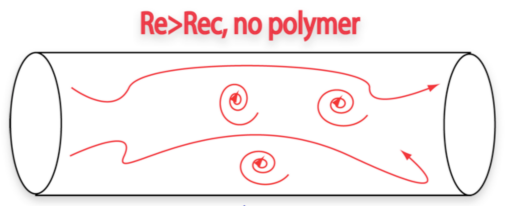

(a)

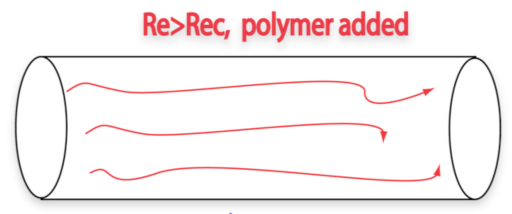

(b)

Figure 6. (a) Sketch of a turbulent pipe flow with no polymer added. (b) Sketch of a turbulent pipe flow after a small amount of polymer has been added.

The overall picture is dramatically different if the same experiment is performed with a polymeric fluid, Fig. 5(b). The diameter of the exiting fluid jet $D_{P}$ may be up to three times larger than the diameter of the capillary. This phenomenon is called the "extrudate swell". A simplified phenomenological interpretation of this effect may be given if one resorts once again to the original idea of Weissenberg of tension generation along the stream lines in flows of polymeric fluids. When the fluid exits the capillary this extra tension can not be accommodated and, as a result, the fluid will contract axially and expand radially.

A more rigorous and quantitative result which relates the swelling ratio $\frac{D_{P}}{D}$ to the ratio between the first normal stress difference $N_{1}$ shear stress measured near the wall at the capillary exit was given by Tanner, Tanner [1970]:

$$
\frac{D_{P}}{D}=0.1+\left[1+\frac{1}{2}\left(1+\left(\frac{N_{1}}{\sigma_{x y}}\right)_{w}^{2}\right)\right]^{1 / 6}
$$

\subsection{Drag reduction}

In 1949 Toms discovered that a small addition of a flexible linear polymer (roughly $10 \mathrm{ppm}$ of polymethylacrylate) to an inertially turbulent leads to a substantial reduction of the turbulent drag (defined by the pressure drop $\Delta P$ needed to maintain a given flow rate) that may reach $40 \%$, Toms [1949]. This phenomenon has been coined the drag reduction phenomenon.

The early studies of drag reduction in dilute polymer solutions revealed two types of drag reduction in dilute polymer solutions, Hershey and Zalkin [1967]. In dilute polymer solutions the drag reduction emerges in a fully developed turbulence regime beyond a critical Reynolds number where the 
friction factor decreases below that for an ordinary Newtonian turbulent flow. For Reynolds numbers smaller that the onset value, no drag reduction is observed. In more concentrated polymer solutions, the drag reduction is observed near the upper bond of the laminar region. In this case, onset conditions are reached at low Reynolds numbers, that is, the laminar-turbulent transition is not observed. The main difference between these two kinds of drag reduction is the region where drag reduction occurs. The former begins in the fully developed turbulent region. The latter is observed in the extended laminar region. They were later labeled type A and type B drag reduction by Virk and Wagger, Virk and Wagger [1990]. Despite a large number of theoretical, numerical and experimental studies ?Yarin [1997] that span almost six decades, the mechanism by which the polymer addition inhibits the momentum transfer to the channel walls and the drag reduction occurs it is still not fully understood.

\section{$3 \quad$ Hydrodynamic stability of dilute polymer solutions}

Vinogradov and Manin [1965]

Stretching of individual polymer molecules in the flow leads to generation of additional elastic stresses that relax with a macroscopic characteristic time $\lambda$, are flow history dependent and grow in a strongly nonlinear fashion with the external forcing. Thus, the stress tensor, $\tau$, can be decomposed in two parts, $\tau=\tau_{s}+\tau_{p}$. The first term $\tau_{s}$ is proportional to the viscosity of the newtonian solvent, $\eta_{s}$, and to the rate of strain in the flow

$$
\tau_{s}=-\eta_{s} \cdot\left[\nabla \vec{v}+(\nabla \vec{v})^{T}\right]
$$

Therefore, the equation of motion of a dilute polymer solution becomes

$$
\partial_{t} \vec{v}+\vec{v} \nabla \vec{v}=-\frac{\nabla p}{\rho}+\frac{\eta_{s}}{\rho} \triangle \vec{v}-\frac{\nabla \tau_{p}}{\rho}
$$

Here the elastic stress tensor, $\tau_{p}$ is due to the presence of polymer molecules which are stretched in the flow. The simplest model that accounts for the elastic nature of the polymer part of the stress tensor, $\tau_{p}$, is a Maxwell type constitutive equation with a single relaxation time, $\lambda$,

$$
\tau_{p}+\lambda \cdot \frac{D \tau_{p}}{D t}=-\eta_{p}\left[\nabla \vec{v}+(\nabla \vec{v})^{T}\right]
$$

Here $\frac{D \tau_{p}}{D t}$ is the upper convective time derivative defined by

$$
\frac{D \tau_{p}}{D t}=\frac{\partial \tau_{p}}{\partial t}+(\vec{v} \nabla) \cdot \tau_{p}-(\vec{v} \nabla)^{T} \cdot \tau_{p}-\tau_{p} \cdot(\nabla \vec{v})
$$


Equations (4), (5) and (2) constitute the Oldroyd-B model of polymer solution rheology Bird et al. [1977]. It is worth noting that the non-linear terms involved in the equations (4) and (5) are all of the order $\lambda\left(\frac{V}{L}\right) \tau_{p}$. The balance between the non linear elastic terms and the linear dissipative terms is quantified by a second dimensionless quantity usually referred as the Weissenberg number, $W i=\lambda \cdot \frac{v}{L}$. The mechanical properties of a dilute polymer solution become increasingly nonlinear as $W i$ is increased.

Few effects driven by the nonlinear polymer stress have been understood long time ago Bird et al. [1977].

For example, in a simple shear flow of a dilute polymer solution, there is always a difference between the normal stresses along the flow-line and orthogonal to it. In the limit of small shear rates, this normal stress difference, $N_{1}$, is proportional to the square of the shear rate. In the case of flows with curvilinear streamlines, the normal force difference gives rise to a volume force acting on the fluid elements in the direction of the curvature, hoop stress. Thus, if a rotating rod is inserted in an open container filled with a polymer solution, the fluid climbs the rod instead of being pushed outwards ,Bird et al. [1977]. This phenomenon is known as rod climbing or Weissenberg effect, ?. Secondly, the resistance in a purely extensional flow of a dilute polymer solution increases with the rate of extension in a strongly nonlinear manner. When the rate of extension becomes larger than $\frac{1}{2 \cdot \lambda}$ (which corresponds to $W i>\frac{1}{2}$ ) a sharp growth of the elastic stresses is observed. As a result, the apparent viscosity of a dilute polymer solution can increase up to few orders of magnitude Tirtaatmadja and Sridhar [1993].

One should point out here that both the Weissenberg effect and the nonlinear growth of resistance in extensional flow have been observed in highly viscous polymer solutions at quite low $R e$, that is in the absence of any inertial nonlinear effects. Thus, these effects are solely due to the nonlinear elastic stresses.

In the context of two potential sources of nonlinearity in the hydrodynamic equations of a dilute polymer solution, inertial and elastic, a natural question arises: how is the hydrodynamic stability affected by each nonlinear contribution. This question can be only partially answered. Figure 7 shows a sketch of a stability diagram in the parameter space $(W i-R e)$. If both $R e$ and $W i$ are smaller than unity, the flow is steady and laminar. In the limit of negligible $W i$, the flow looses its stability only if the inertial nonlinearity is large comparative to the viscous dissipation. Thus when Reynolds number exceeds a threshold value, $R e>R e_{c}$, the flow evolves to turbulent states (the region IT in Fig. 7). This is, of course, an oversimplified picture. In most of the realistic situations, the transition to fully 


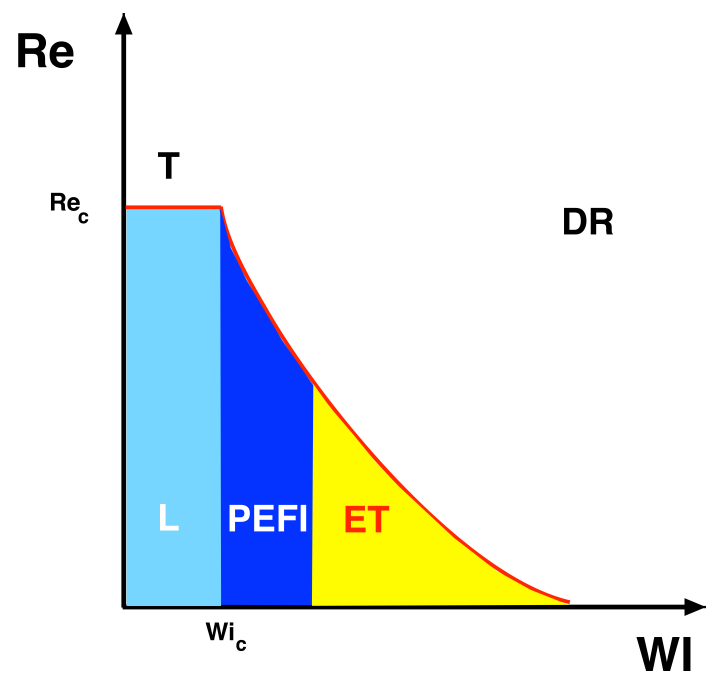

Figure 7. Sketch of hydrodynamic stability diagram: T-inertial turbulence, ET-elastic turbulence, DR-drag reduction, PEFI-purely elastic flow instabilities.

developed turbulence does not occur directly but via secondary instabilities. When $R e$ is small but the values of $W i$ slightly exceed unity, the flow can undergo purely elastic instabilities, Muller et al. [1989a], Larson [1992]. As a result of these instabilities, secondary flows have been observed experimentally Muller et al. [1989b] together with an increase in flow resistance Magda and Larson [1988].

More recently, Groisman and Steinberg have shown that, corresponding to values of $W i$ significantly larger than unity and arbitrarily small values of $R e$, the flow evolves towards random states in a regime of so called elastic turbulence Groisman and Steinberg [2000]. This corresponds to the region (ET) shown in Fig. 7. The elastic turbulence is a chaotic dynamic state solely driven by nonlinear elastic stresses. Although it can be excited in the absence of inertial effects, it exhibits few of the main features of the high $R e$ turbulence: significant increase in the rates of momentum and mass transfer (flow resistance and mixing), chaotic fluid motion excited in a broad range of spatial and temporal scales. However, there are also major differences with respect to the case of inertial turbulence. The main difference is probably the absence of any analogue of the Kolmogorov scale: although random in 
time, the flow is smooth at all spatial scales. The energy is mainly pumped by the large scale flow field and the leading mechanism for generation of small scale structures is the passive advection of the elastic stresses.

One of the most remarkable hydrodynamic effects caused by a small addition of polymer molecules to a high Re turbulent flow is the drag reduction. In 1948, Toms Toms [1949] discovered that a minor addition of polymethylmethacrylate (about 10ppm by weight) to a turbulent pipe flow results in a significant reduction in pressure drop needed to drive the flow at the given flow rate. The tremendous difficulty in understanding the drag reduction originates in the fact that it occurs at both very high $R e$ and $W i$ (the region DR in Fig. 7) and thus, one has to deal simultaneously with two strong nonlinearities in the flow: inertial and elastic.

The work presented in this thesis mainly focuses on two regions of the stability diagram sketched in Fig. 7: ET and PEFI. I have tried to organise the material presented in a self-consistent manner that allows an independent reading of each Chapter. It is organised as follows.

In Chapter 1 an experimental investigation of the elastic turbulence in a swirling flow configuration is presented. Chapter 2 follows with a detailed investigation of random micro-flows of a dilute polymer solution in a regime of elastic turbulence. Chapter 3 presents a theoretical and numerical investigation of purely elastic waves sustained by a stratification of elastic stresses in curvilinear geometries.

\section{Elastic turbulence in dilute polymer solutions: turbulence without inertia}

The addition of a tiny amount of high molar mass linear polymer molecules to a fluid makes it elastic and capable of storing stresses that depend on the flow history, Bird et al. [1977]. The polymer contribution to the stress tensor depends on the flow forcing in a strongly nonlinear fashion and may lead to a loss of the hydrodynamic stability of shear flows with curvilinear trajectories Larson [1992], Shaqfeh [1996], Muller et al. [1989a]. The purely elastic instabilities are triggered in the absence of any inertial contributions (i.e. at vanishingly small Reynolds numbers, $R e$ ) when the elastic energy overcomes the dissipation due to polymer relaxation. The ratio of the nonlinear elastic term to the linear relaxation one is defined by the Weissenberg number $W i$ which is the main control parameter and the purely elastic instabilities in curvilinear shear flows are observed when $W i>W i_{c}$. A further increase of $W i$ past the onset of the primary elastic instability leads to the observation of the so called "Elastic Turbulence", Groisman and Steinberg [2000]. The elastic turbulence is a random flow state solely driven by the nonlinear 
elastic stresses and, as opposed to its inertial counterpart, it can be observed at arbitrarily small Re.

Following the discovery of the elastic turbulence, a number of experimental studies have revealed several of its key features in various flow macroscopic configurations Burghelea et al. [2007, 2004b], Burghelea, T et al. [2005], Burghelea, Teodor et al. [2006].

Below we detail several of the main features of the elastic turbulence observed in a macroscopic flows with curved streamlines - a vo,n Karman swirling flow.

\subsection{Methods}

Experimenta apparatus The experimental apparatus is schematically shown in Fig. 8. It consists of a stationary cylindrical container, $\mathbf{C}$, (with radius $R_{c}$ ) mounted on the shaft of a commercial rheometer (AR-1000 from TA Instruments) and a disk, $\mathbf{D}$, (with radius $R_{d}$ ) mounted concentrically on the shaft of the rheometer. In order to check the sensitivity of the results with respect to the geometrical aspect ratio and to explore a broad range of Weissenberg numbers, two versions of this setup have been used: first one had $R_{c}=2.2 \mathrm{~cm}, R_{d}=2 \mathrm{~cm}$ and the distance between plates $d=1 \mathrm{~cm}$ and the second had $R_{c}=4.9 \mathrm{~cm}, R_{d}=4.7 \mathrm{~cm}$ and $d=1 \mathrm{~cm}$. The two setups will be further referred as setup 1 and setup 2. The rheometer can be driven either in constant (within $0.5 \%$ ) angular speed mode either in constant(within $0.5 \%$ ) torque mode. The two forcing modes will be further referred as $\Omega$-forcing and $T$-forcing. The momentum of inertia of the shaft of the rheometer is $I_{s} \approx 14 \mu \mathrm{Nms}^{2}$ and the momentum of inertia of the upper plate, $I_{d}$, was about $61 \mu N m s^{2}$ for setup 1 , and about $84 \mu N m s^{2}$ for setup 2 . The accuracy of the angular speed measurements in constant torque mode is about $2 \%$ and the accuracy of the torque measurements in the constant speed mode is about $1 \%$. One has to point out here that smallness of the fluctuations rate of the angular velocity is not a sufficient criterion to have a constant speed forcing. Corresponding to the $\Omega$-mode, $\left(I_{r}+I_{d}\right) \frac{\partial \Omega}{\partial t}$ should be also much smaller than the typical values of torque, $T$.

The system was illuminated laterally by a thin $(30 \mu \mathrm{m}$ in the centre of the setup and about $120 \mu \mathrm{m}$ at the edges of the setup) laser sheet at the middle distance between plates. The laser sheet was generated by passing a laser beam delivered by a $300 \mathrm{~mW}$ argon-ion laser, $\mathbf{L}$, through a block of two crossed cylindrical lenses, $\mathbf{C O}$, mounted in a telescopic arrangement. Flow images are acquired with a digital camera (PixelFly from PCO with 12 bit quantisation and $640 \times 512$ pixels resolution)from below, through a 45deg flat mirror, $\mathbf{M}$. 


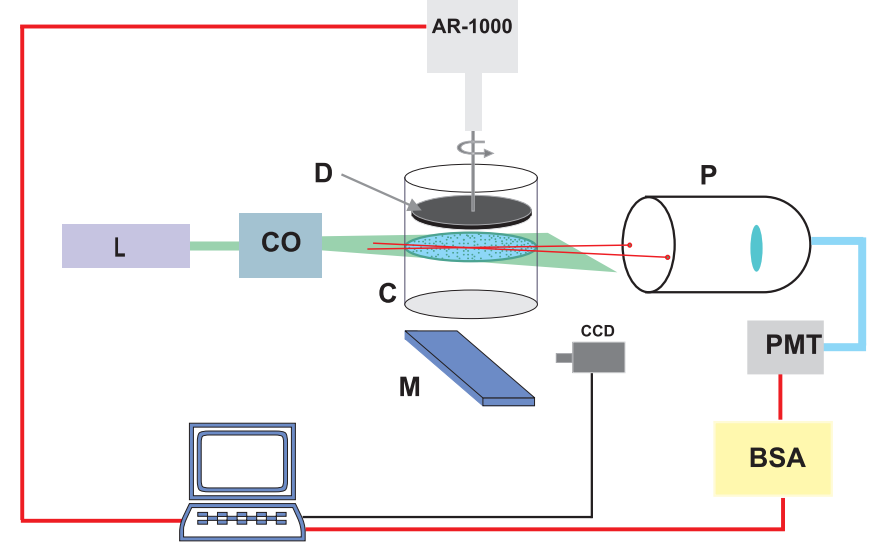

Figure 8. Overview of the swirling flow apparatus: C- fluid container, D-rotating disk ,AR-1000-rheometer, CO-cylindrical optics, L- laser, M- plane mirror, CCD-digital camera, P-LDV probe,BSA-burst spectrum analyser, PMT-photomultiplier.

Rheological properties of the polymer solutions The polymer used was polyacrylamide, PAAm (from Polysciences Inc.) with the molecular weight $M_{w}=1.8 \times 10^{7} \mathrm{Da}$ which was the same polymer used in Ref. Groisman and Steinberg [2001]. The polymer was dissolved at a concentration of $80 \mathrm{ppm}$ in a Newtonian solvent. The Newtonian solvent was about $65 \%$ saccharose in water.

The rheological properties of the solvent and the polymer solution were measured with two different rheometers: AR-1000 from TA Instruments and Vilastic 3 from Vilastic Scientific. The viscosity of the solvent was $\eta_{s}=114 \mathrm{mPas}$ at $22^{\circ} \mathrm{C}$, and the viscosity of the solution was $\eta=138 \mathrm{mPas}$ at a shear rate of $2 s^{-1}$. The polymer relaxation time, $\lambda$, was measured in oscillatory tests at different shear rates, $\dot{\gamma}$, ranging from $0.4 s^{-1}$ to $3.6 s^{-1}$. The real and imaginary part of the complex viscosity (or the components inphase and out-of-phase with the applied shear), $\eta^{\prime}$ and $\eta^{\prime \prime}$, respectively, were measured in long series at different angular frequencies ranging from $0.1 \mathrm{~Hz}$ to $1 \mathrm{~Hz}$. Corresponding to each frequency, the results were averaged over six different runs. The same procedure was applied with the solvent ant its viscosity components, $\eta_{s}^{\prime}$ and $\eta_{s}^{\prime \prime}$ were measured as well. The values for the polymer in-phase and out-of-phase viscosity were calculated as $\eta_{p}^{\prime}=\eta^{\prime}-\eta_{s}^{\prime}$ and $\eta_{p}^{\prime \prime}=\eta^{\prime \prime}-\eta_{p}^{\prime \prime}$. The polymer relaxation time was finally calculated 


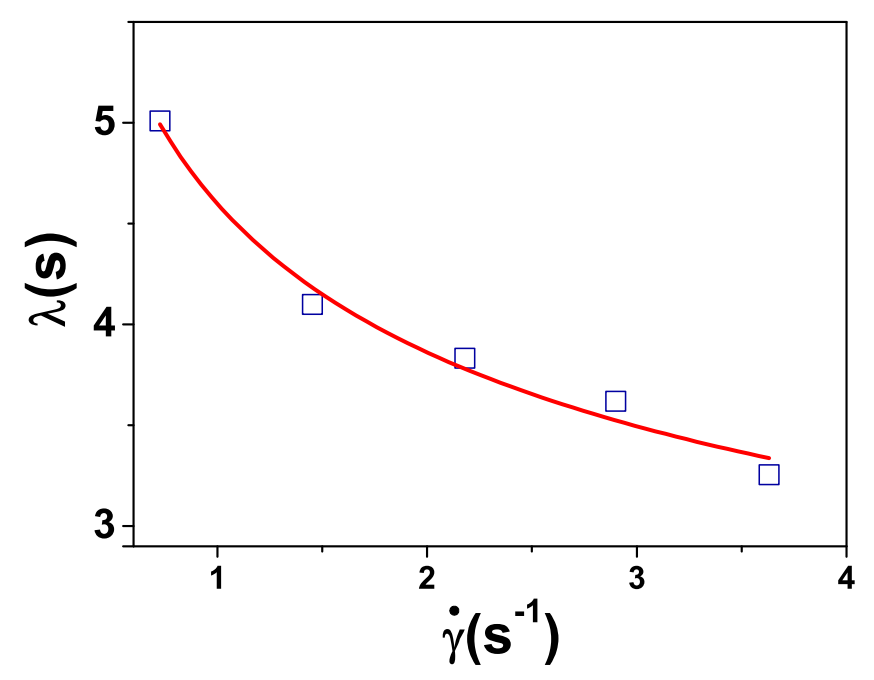

Figure 9. Polymer relaxation obtained from oscillation measurements in a Mooney-Ewart geometry mounted on the AR-1000 rheometer. The full line is a fit $\dot{\gamma}^{-0.29}$.

$\lambda=\lim _{\omega \rightarrow 0}\left[\frac{1}{\omega}\left(\frac{\eta_{p}^{\prime \prime}(\omega)}{\eta_{p}^{\prime}(\omega)}\right)\right]$.

The dependence of the polymer relaxation time on the shear rate is presented in Fig. 9.

Flow field measurements In order to obtain a detailed characterisation of both spatial structure and temporal evolution of the flow-fields, three different experimental techniques have been alternatively used: particle image velocimetry (PIV), particle tracking velocimetry (PTV) and laser Doppler anemometry (LDA). As flow tracers we have used $10 \mu m$ fluorescent particles, for the PIV and PTV measurements and $1 \mu m$ latex beads for the LDA measurements.

We have acquired 2000 pairs of flow images every $120 \mathrm{~ms}$. A custom development of the camera software allowed us to adjust the time delay between consecutive images in relation with the local flow velocity (in order to keep the mean particle displacement in the range $5-15$ pixels). Corresponding to low values of the angular velocity of the upper disk (up to about $0.4 \mathrm{rad} / \mathrm{s}$ ) the time delay was $540 \mathrm{~ms}$ and then, gradually decreased down to $40 \mathrm{~ms}$. The total data acquisition time was always longer than 
the Eulerian correlation times of the velocity (about 240 times in the fully developed random regime). Time series of velocity fields are obtained by a multi-pass PIV algorithm. The space resolution was $0.89 \mathrm{~mm}$ in setup 2 and $0.57 \mathrm{~mm}$ in setup 1 . The accuracy of the method has been carefully checked by running test experiments with the solvent, in the same range of mean particle displacements and at similar illumination conditions. Although an instrumental error increased more or less linearly with the mean particle displacement, it has never exceeded $5 \%$ of the mean displacement. By post processing the velocity fields, we obtain the profiles of the velocity components, fields of fluctuations of each velocity component, space spectra of the velocity fluctuations, velocity gradients and their fluctuations, structure functions of gradients, power spectra of velocity fluctuations, eulerian velocity correlation times. The space-time measurements together with simultaneous bulk measurements of the flow resistance provide a rather complete description of the different flow regimes as a function of $W i$.

In order to check the consistency of the PIV approach (particularly when Lagrangian trajectories were measured), flow fields have been alternatively investigated by PTV technique. The first step of the PTV approach is an accurate particle identification in each flow image, by correlation with prototypical particle shapes which can be either extracted from flow images, either defined manually. Secondly, trajectories are reconstructed by joining successive positions of the same particle on subsequent images. This has been done by a search algorithm based on an initial "guess" of the mean flow line. Although the PIV and PTV tools are very suitable for the investigation of the spatial properties of the flow, they provide a limited time resolution and statistics. In order to overcome this limitation, the LDA technique has been alternatively used. By the $L D A$ technique, time series of the fluid velocity are measured in a relatively small volume (typically $100 \mu \mathrm{m} \times$ $50 \mu \mathrm{m} \times 50 \mu \mathrm{m})$. A commercial $L D A$ system from Dantec Dynamics Inc. has been used. Two laser beam delivered by a probe (P) (Fig. 8)are aligned to cross each other at an angle of $\theta=10 \mathrm{deg}$ in some point in the flow. The light scattered in the backward direction by the flow tracers present in the flow is collected by the probe $\mathbf{P}$ and delivered to a photomultiplier, PMT via a fiber optic light guide. The PMT signal is processed by a real time burst spectrum analyser BSA. The local flow velocity is proportional to the Doppler shift of the signal. The error validated velocity data is stored on a computer via a GPIB line. 


\subsection{Flow resistance}

One of the main features of the transition to elastic turbulence is the substantial growth of flow resistance above the onset of the instability Groisman and Steinberg [2000], Burghelea et al. [2007, 2004c]. In the case of a von Karman swirling flow, a measure of the flow resistance is either the power needed to spin the upper plate a constant angular speed, $P_{\Omega}$, either the power needed to spin the upper plate in a regime of constant torque, $T$, on the shaft of the rheometer, $P_{T}$. For a swirling flow between parallel plates, the Reynolds number can be defined as $R e=\frac{\Omega \cdot R_{c} \cdot d \cdot \rho}{\eta}$. In our experiments the inertial contribution was always low, $R e<16$. The relevant control parameter is the Weissenberg number, $W i=\lambda(\dot{\gamma}) \cdot \dot{\gamma}$ where the shear rate, $\dot{\gamma}$, can be estimated ${ }^{1}$ as $\dot{\gamma}=\frac{\Omega \cdot R_{c}}{d}$.

The dependencies of the injected power and of the rms of its fluctuations on the control parameter, $W i$, are presented in Fig. $10 \mathrm{~A}, \mathrm{~B}$.

The data presented in Fig. 10 A,B reveal three distinct flow regimes. For low values of the control parameter, $W i$, the injected power grows as $P_{\text {lam }} \propto W i^{2.85}$. If one takes into account the thinning of the polymer relaxation time with the shear rate (Fig. 9), one can easily conclude that the power grows quadratically with the angular speed, consistent with a linear and laminar flow regime. Corresponding to $W i \approx W i_{c}$, a primary elastic instability occurs, resulting in a sharp increase of the injected power and of the rms of its fluctuations. Increasing further the control parameter, the flow evolves towards a scaling regime $\left(W i>W i_{e t}\right)$ where both the average and the rms of fluctuations of the injected power scale algebraically with $W i$ (Fig. 10): $P \propto W i^{3.34}$ and $P_{r m s} \propto W i^{3.21}$. The scaling of the mean injected power with the control parameter can be explained by simple considerations. Due to the smallness of the inertial nonlinearity in the flow $(R e<16)$, the relative growth of the mean injected power with respect to its laminar values, should be solely due to the mean elastic contribution to the stresses, $\frac{P}{P_{\text {lam }}} \propto \tau_{p}$. Therefore, according to the fit presented in Fig. 10 $\mathrm{A}, \tau_{p} \propto W i^{0.49}$.

According to theory, Balkovsky and Fouxon [1999], Balkovsky et al. [2001], for a sufficiently large degree of stretching of the polymer molecules in the flow (when the back reaction of the polymer molecules is switched on), the elastic contribution to the stress tensor, $\tau_{p}$, scales as the inverse polymer relaxation time, $\tau_{p} \propto \lambda^{-1}$. Next, if one takes into account the dependence of the relaxation time on the shear rates presented in sub section 1.1.1, $\lambda \propto \dot{\gamma} \cdot{ }^{-0.3}$, one obtains $\tau_{p} \propto W i^{0.43}$ which is rather close to the value

\footnotetext{
${ }^{1}$ Due to the strong non homogeneity of the shear rates in a swirling flow, even in laminar regime, this choice is somewhat arbitrary.
} 

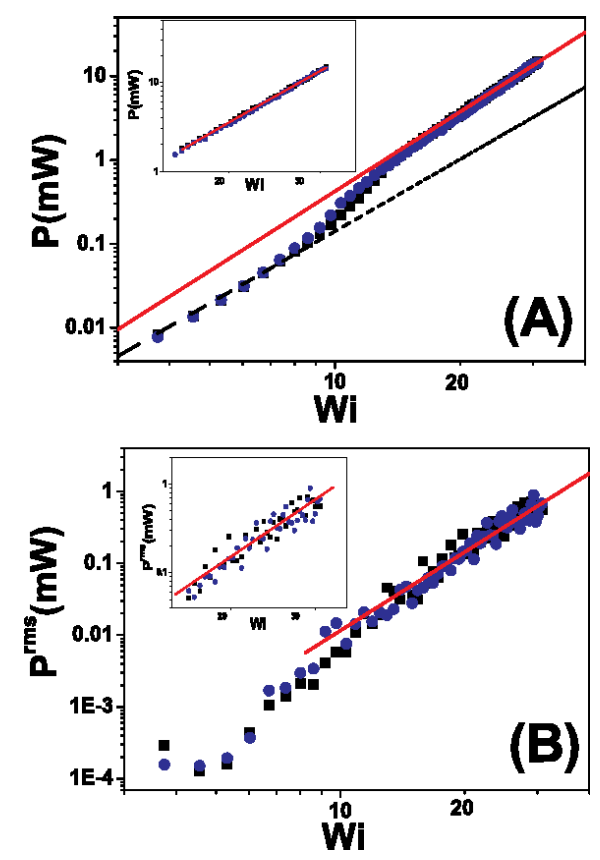

Figure 10. (A)Dependence of the mean power, $P$, on the control parameter, $W i$ : squares-increasing $W i$, circles-decreasing $W i$. The full line is a guide for the eye, $W i^{3.34}$. The dotted line is $W i^{2.85}$. The inset shows a power law fit, $W i^{3.34 \pm 0.05}$ (B) Dependence of the rms of power fluctuations, $P^{r m s}$, on $W i$ : squares-increasing $W i$, circles-decreasing $W i$. The full lines are power laws, $W i^{3.21}$. The inset shows the power law fits, $W i^{3.21 \pm 0.3}$. Data were collected in setup 2 in $\Omega$ forcing mode. 


\begin{tabular}{||c|c|c|c|c|c|c|c|c|c|c||}
\hline Setup & $\epsilon$ & $\Omega_{c}(\mathrm{rad} / \mathrm{s})$ & $\Omega_{e t}(\mathrm{rad} / \mathrm{s})$ & $\gamma_{c}\left(\mathrm{~s}^{-1}\right)$ & $\gamma_{e t}\left(\mathrm{~s}^{-1}\right)$ & $\lambda_{c}(s)$ & $\lambda_{e t}(s)$ & $W i_{c}$ & $W i_{e t}$ & $R e_{c}$ \\
\hline 1 & 0.5 & 1.3 & 2 & 2.6 & 4.6 & 3.4 & 3 & 10 & 13.8 & 2.7 \\
\hline 2 & 0.2 & 0.3 & 0.97 & 1.43 & 4.74 & 4.2 & 2.95 & 6 & 14 & 1.38 \\
\hline
\end{tabular}

Table 1. Onset values of the relevant physical quantities corresponding to the primary elastic instability and fully elastic turbulent regime.
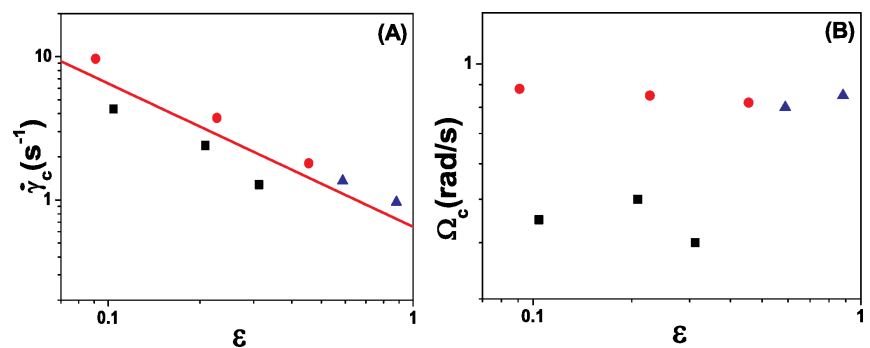

Figure 11. Dependence of the onset of the primary elastic instability on geometric aspect ratio:(A) Shear Rate (B) Angular velocity. The symbols are: squares- $R_{d}=4.7 \mathrm{~cm}$, circles- $R_{d}=2.2 \mathrm{~cm}$, triangles- $R_{d}=1.7 \mathrm{~cm}$. The full line is $\epsilon^{-1}$.

0.49 obtained experimentally. The onset values of the angular speed and the corresponding values of the shear rate, characteristic relaxation time and $W i$ are presented in the table 1.

Corresponding to the onset of the elastic instability, $R e$ was of order of unity in both setups, suggesting that the transition is of purely elastic nature.

One has to point out a discrepancy between the values of the geometric aspect ratio $\epsilon=d / R_{c}$ for each of the setups used and the corresponding onset values, $W i_{c}$, for the primary elastic instability. Indeed, as illustrated in the table above, although the first setup has a larger aspect ratio than the second, the elastic instability occurs here at higher $W i$ than in the second setup. This discrepancy motivated me to study more systematically the relation between the onset of the primary elastic instability and the geometric aspect ratio $\epsilon$. By modifying both the radius of the container, $R_{c}$, and the distance between plates $d$, the geometric aspect ratio $\epsilon$ has been varied about 10 times. For each value of $\epsilon$, the onset of the primary elastic 
instability has been identified by measuring the dependence of the mean injected power on the angular speed of the upper plate. As illustrated in Fig. $11 \mathrm{~A}, \mathrm{~B}$ the onset values of the shear rate scale as $\epsilon^{-1}$ and, implicitly, the onset values of the angular speed are practically independent on the aspect ratio. However, as one can see in $11 \mathrm{~B}$, the onset speeds seem to depend on the radius of the cylindrical container.

One has to point out that these experimental results clearly disagree with the linear stability analysis presented in [8-10] which leads to $\dot{\gamma}_{c} \propto \epsilon^{-\frac{1}{2}}$ and $\Omega_{c} \propto \epsilon^{\frac{1}{2}}$.

Typical time series of the injected power and their probability distribution functions(pdf's) for different $W i$ are displayed in Fig. 12 ( $\Omega$ forcing) and Fig. 13 ( $T$ forcing). For each value of the control parameter, the statistics of the power fluctuations was carried out on 180000 data points evenly sampled in time $\left(t_{s} \approx 0.038 s\right)$.

In order to avoid the mechanical degradation of the polymer solution during long data acquisition times, separate experiments have been conducted for each value of the control parameter. The local(in time) average values of the injected power did not change significantly during the total data acquisition times, suggesting that no major degradation has occurred 2 . For low values of the control parameter, $\left(W i<W i_{c}\right)$, the power fluctuations are only due to the instrumental noise suggesting a linear and laminar flow regime. Above the onset of the elastic instability, the power starts to fluctuate quite randomly in time. Corresponding to large values of $W i$, the pdf's are strongly non Gaussian for both forcing modes. It is worth noting the left side skewness of the pdf's in Fig. 12 and the right side skewness the pdf's in Fig. 13. Understanding the reasons of this asymmetry requires a future investigation.

To conclude this section, accurate measurements of the power injected in the flow revealed three distinct flow regimes. A linear and laminar regime was observed for $W i<W i_{c}$, followed by transitional regime above the onset of the elastic instability, $W i_{c}<W i<W i_{e t}$, and finally the elastic turbulence regime, $W i>W i_{e t}$, where both the injected power and the rms of its fluctuations scale algebraically with the control parameter. The sharp increase of the flow resistance above the onset of the elastic instability is related to a major reorganization that occurs in the flow.

The next section is dedicated to a detailed investigation of the flow structure in each of the three regimes discussed above.

\footnotetext{
${ }^{2}$ One of the signatures of the mechanical degradation is an apparent decrease in time of the viscosity of the solution, which results in a decrease of the injected power.
} 

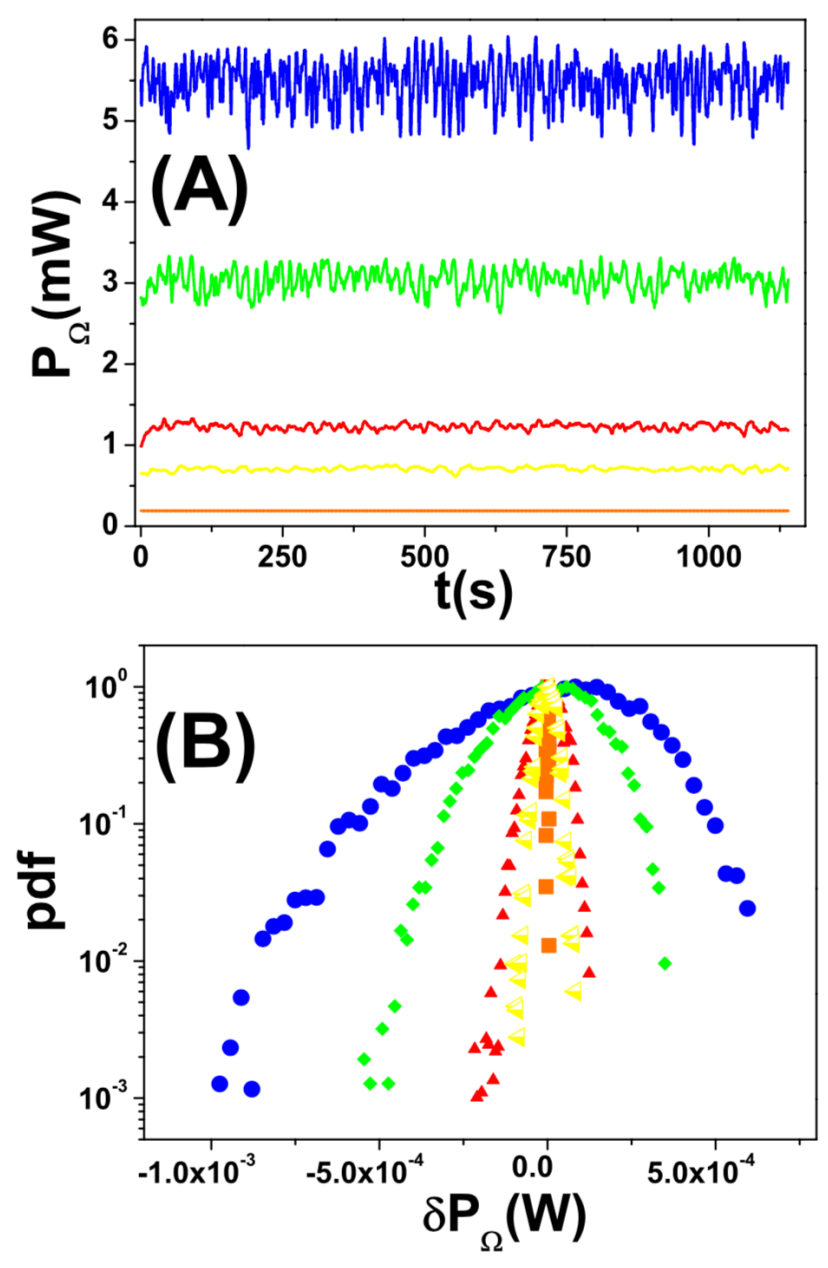

Figure 12. (A) Time series (partially shown) of the injected power in constant $\Omega$ forcing regime for different $W i$. (B)pdf's of the power fluctuations in constant $\Omega$ forcing regime for different $W i$. The colors are: orange- $W i=5$, yellow- $W i=19$, red- $W i=24$, green- $W i=31.5$, blue- $W i=40$. Data were collected in setup 2 . 

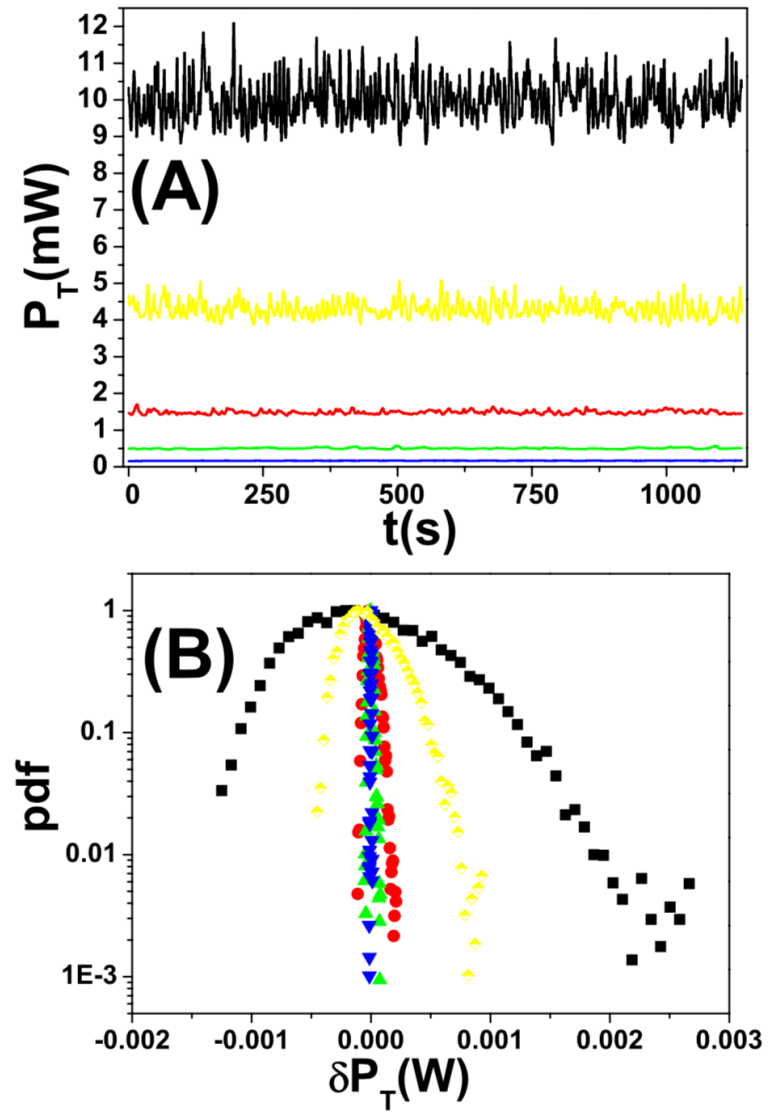

Figure 13. (A) Time series (partially shown) of the injected power in constant $T$ forcing regime for different $W i$. (B)pdf's of the power fluctuations in constant $T$ forcing regime for different $W i$. The colors are: blue- $W i=5$, green- $W i=17$, red- $W i=25$, yellow- $W i=34$, black- $W i=46$. Data were collected in setup 2. 

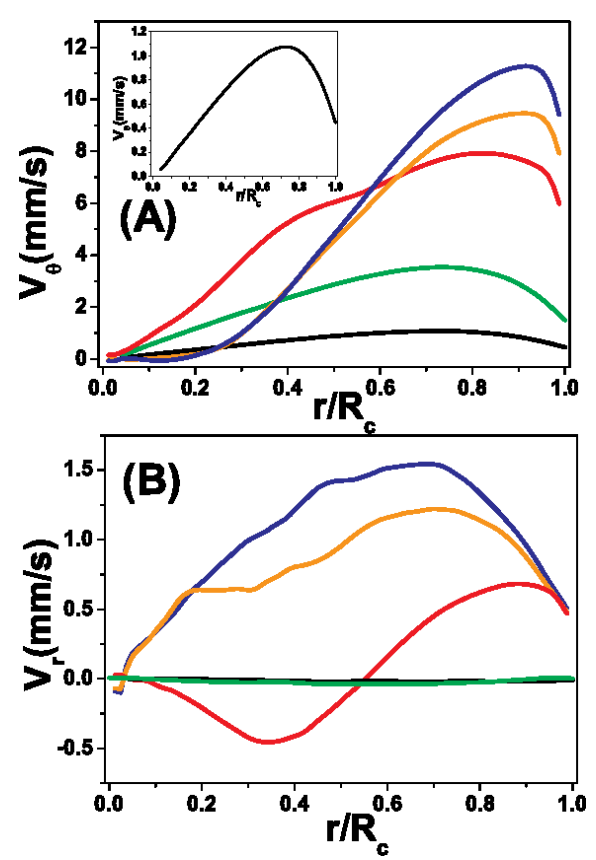

Figure 14. (A) Profiles of the average tangential velocity components, $V_{\theta}$, for several values of $W i$. The inset shows a typical laminar profile of the tangential velocity component. (B)Profiles of the average radial velocity components, $V_{r}$, for several values of $W i$. The colors are: black- $W i=2.48$, green- $W i=4.41$, red- $W i=11.1$, orange- $W i=15$, blue- $W i=18$. Data were collected in setup 1 at middle distance between plates.

\subsection{Flow structure in a regime of Elastic Turbulence}

Corresponding to each value of $W i$ investigated, the flow fields have been characterised by combined PIV, PTV and LDA techniques. Typical time averaged profiles of the tangential, $V_{\theta}$, and radial, $V_{r}$, velocity components are displayed in Fig. 14 A,B. Instantaneous and time averaged velocity fields are presented in Fig. 15(a-c).

Below the onset of the primary elastic instability $\left(W i<W i_{c}\right)$ the flow is steady and laminar. The core region of the flow is consistent with a rigid body rotation characterised by a linear increase of the tangential velocity component(inset in Fig. 14A, Fig. 15(a)) with a slope $\Omega^{-1}$. In the pe- 

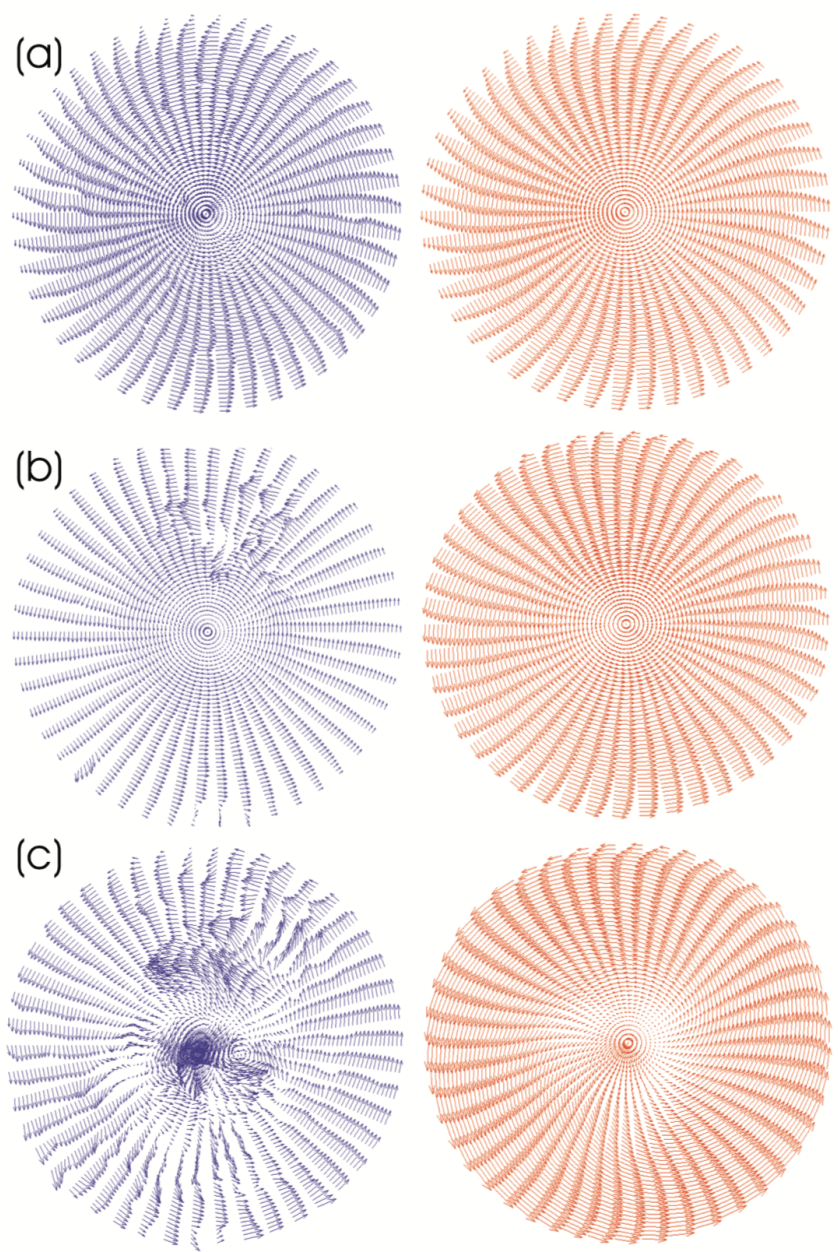

Figure 15. Instantaneous (left column) and time averaged (right column)velocity fields for different values $W i$ : a) $W i=2.48 \mathbf{b}$ ) $W i=9.88$ (c) $\mathrm{Wi}=18.96$. Data were collected in setup 1 at middle distance between plates. 
ripheral region of the flow, $V_{\theta}$ decays towards the boundaries similarly to a Couette flow between cylinders. As illustrated in Fig. 14 B, below the onset of the elastic instability, there is no fluid motion in the radial direction, $V_{r} \approx 0$. This is also illustrated in Fig. 15 (left column), which displays spatial distribution of the radial velocity component for several values of the control parameter. The right column of Fig. 15 displays typical average spatial distributions of the tangential velocity component at different values of $W i$.

The velocity fluctuations in the laminar regime are only due to the instrumental errors and do not exceed $5 \%$ of the mean values (panel (a) in Fig. 17). Corresponding to $W i \approx W i_{c}$, a primary elastic instability driven by the hoop stresses leads to a first flow reorganization ${ }^{3}$ in the form of a large scale structure which is probably similar to the toroidal vortex previously observed experimentally, Groisman and Steinberg [2004] . Large scale toroidal vortices driven by the hoop stresses are actually quite well known to appear in swirling flows of viscoelastic fluids Bird et al. [1977], STOKES et al. [2001a,b] and, the non homogeneity of the shear rate profile in the primary laminar flow has been long recognised as their common origin. The mean radial velocity component changes its sign in the radial direction, from negative values near the centre of the system, to positive ones towards the boundaries(the red curve in Fig. 14B). This sign alternation of the radial velocity component in the radial direction is also apparent in Fig. 15(B).

The first flow reorganisation in the intermediate regime, $W i_{c}<W i<$ $W i_{e t}$, is accompanied by a sharp increase of the level of velocity fluctuations (Figs. 17, 19). The field of fluctuations has a ring shaped topology (Fig. 17 (b-c)) which is probably related to the topology of the secondary flow developed in this region. The toroidal vortex is providing a smooth large scale velocity field (as illustrated in panel Fig. 15(b), the instantaneous velocity field displays no small scale features) which fluctuates randomly in time. The stress tensor is imbedded in this field and chaotically advected by it. Such advection can create small scale fluctuations of stresses which can result in small scale fluid motion ${ }^{4}$ Balkovsky and Fouxon [1999], Fouxon and Lebedev [2003], Balkovsky et al. [2001].

It is worth noting that in the peripheral region of the flow (down triangles, left triangles in Fig. 18), the increase of turbulence intensity defined as defined as $I_{t}=\frac{V_{\theta}^{r m s}}{V_{\theta}}$ occurs at slightly lower values of $W i$ than in the

\footnotetext{
${ }^{3}$ As one can see from Fig. 10, this large scale flow reorganization costs in a drastic increase of both the flow resistance and level of fluctuations of the injected power.

${ }^{4}$ The mechanism is similar to generation of small scale passive scalar variations in the chaotic mixing in spatially smooth random flows.
} 


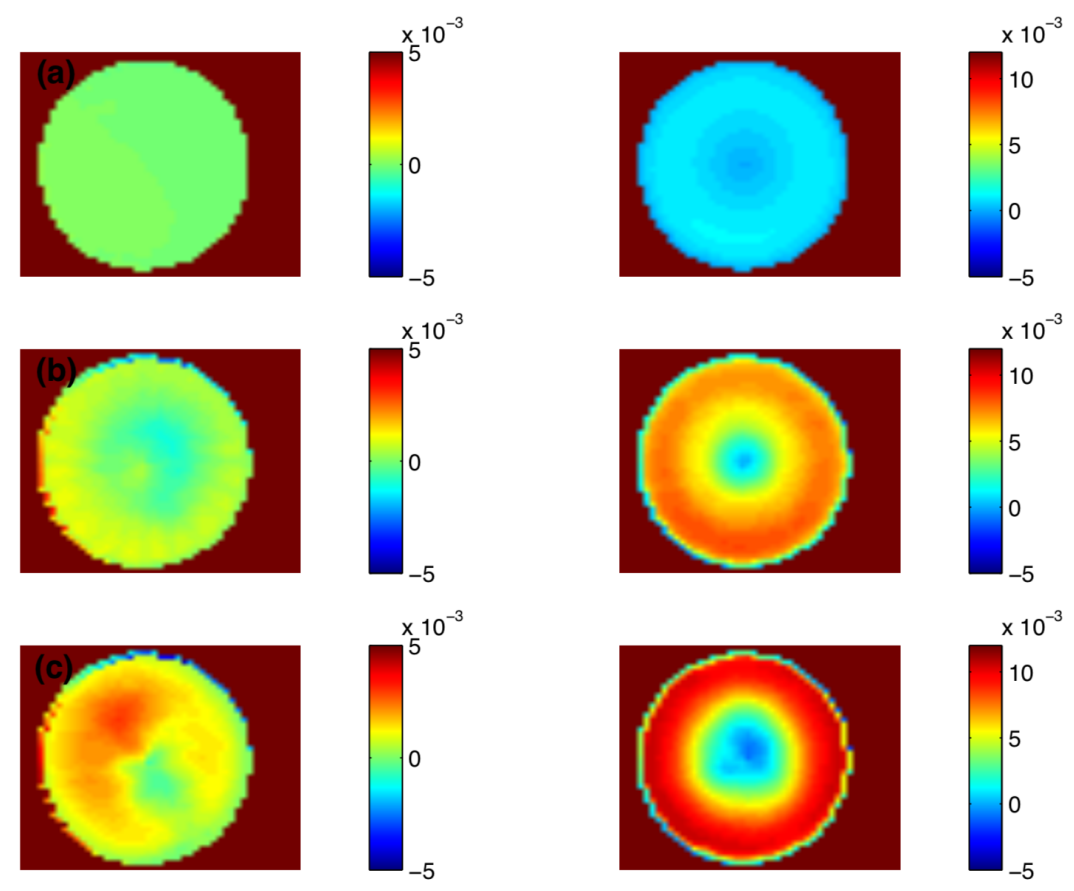

Figure 16. Distribution of the radial velocity component(left column) and of the tangential velocity components ( right column) for different values of $W i$ :(a) $W i=2.48$ (b) $W i=9.88$ (c) Wi $=18.96$. Data were collected in setup 1 at middle distance between plates.

central region (up triangles, circles). Here $V_{\theta}^{r m s}$ is the rms of fluctuations of the tangential velocity component, $V_{\theta}^{r m s}=\left\langle V_{\theta}^{2}\right\rangle_{t}^{\frac{1}{2}}$. This suggests that instability front propagates from the boundary towards the bulk of the flow. The forthcoming analysis of the velocity gradients will clarify this issue. Radial profiles of the turbulence intensity, $I_{t}$ are displayed in Fig. 19.

The large scale toroidal vortex developed in the intermediate region mediates the transition to elastic turbulence regime of Elastic Turbulence, $\left(W i>W i_{e t}\right)$. Corresponding to this regime, a second flow reorganisation occurs ${ }^{5}$. As suggested by panel (c) in Figs. 15, 16, the dominant flow structure is now a large scale spiral vortex. The topology of the field of

\footnotetext{
${ }^{5}$ Corresponding to this second flow reorganization, both the mean injected power and
} 

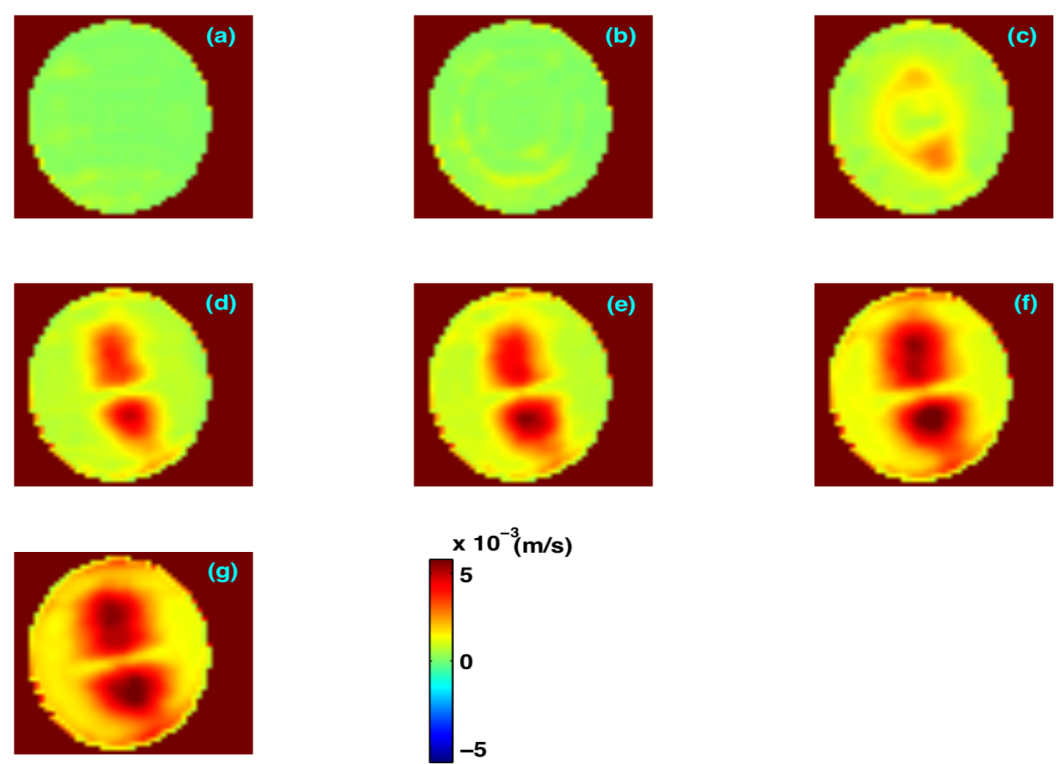

Figure 17. Fields of fluctuations of the tangential velocity component at different $W i$ :(a) $W i=8.32$.(b) $W i=9.88$.(c) $W i=11.1$.(d) $W i=$ 12.72.(e) $W i=13.83$.(f) $W i=17(\mathrm{~g}) W i=19$. Data were collected in the setup 1 at middle distance between plates.

fluctuations also changes drastically. In setup 1 , the circular symmetry (see the ring like structure in panels (b),(c) in Fig. 17) is broken, and the fluctuation fields have now a dipolar appearance (panels (d)-(g) in Fig. 17). A different ${ }^{6}$ topological change has been observed in setup 2, from a ring (in the intermediate region) to a corona in the elastic turbulence region. Figure 1.12 shows radial profiles of the turbulence intensity for several values of the control parameter. The dependence of the turbulence intensity, $I_{t}$, on the control parameter enters a scaling regime, $I_{t} \propto W i^{0.5}$ (Fig. 18, the inset in Fig. 19).

\footnotetext{
the rms of its fluctuations enter a scaling region, Fig. 10.

${ }^{6}$ The difference in the topologies of the fields of fluctuations observed in the two setups is probably related to the sensitivity to the boundary conditions.
} 


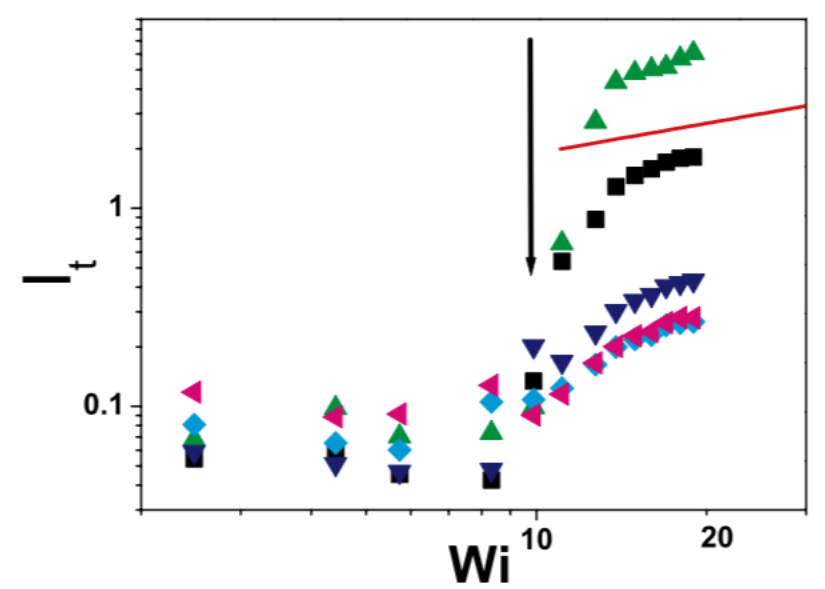

Figure 18. Turbulence intensity versus $W i$ at different radial positions: squares- $r=1 \mathrm{~cm}$, up triangles- $r=0.7 \mathrm{~cm}$, down triangles- $r=1.5 \mathrm{~cm}$, rhumbs- $r=1.74 \mathrm{~cm}$, left triangles- $r=1.89 \mathrm{~cm}$. The arrow indicates the onset of the elastic instability. The full line is a guide for the eye, $W i^{0.5}$. The data were collected in setup 1.

The Eulerian correlation time can be defined as

$$
\tau_{c}=\int t C(t) d t / \int C(t) d t
$$

using the Eulerian correlation function (data are not shown here) of the velocity in a regime of elastic turbulence, $C(t)$.

The transition to elastic turbulence is accompanied by a significant decrease of the correlation times, $\tau_{c}$, from values of order of $15 \mathrm{~s}$ in the intermediate flow regime to values of about $1 s$, Fig. 20. An apparent saturation of the correlation time in the elastic turbulence regime was observed in both setups, suggesting that some sort of dynamical equilibrium is reached. The saturation level is of the order of $1 / \lambda$, Fig. 9.

A main similarity between random flows in a regime of elastic turbulence and high $R e$ turbulent flows is the fast decay of the power spectra of velocity fluctuations Groisman and Steinberg [2000, 2001, 2004].

Fig. 21 displays space ${ }^{7}$ spectra of the velocity fluctuations for different

\footnotetext{
${ }^{7}$ The spectra were obtained from the time dependent measurements of the flow fields
} 


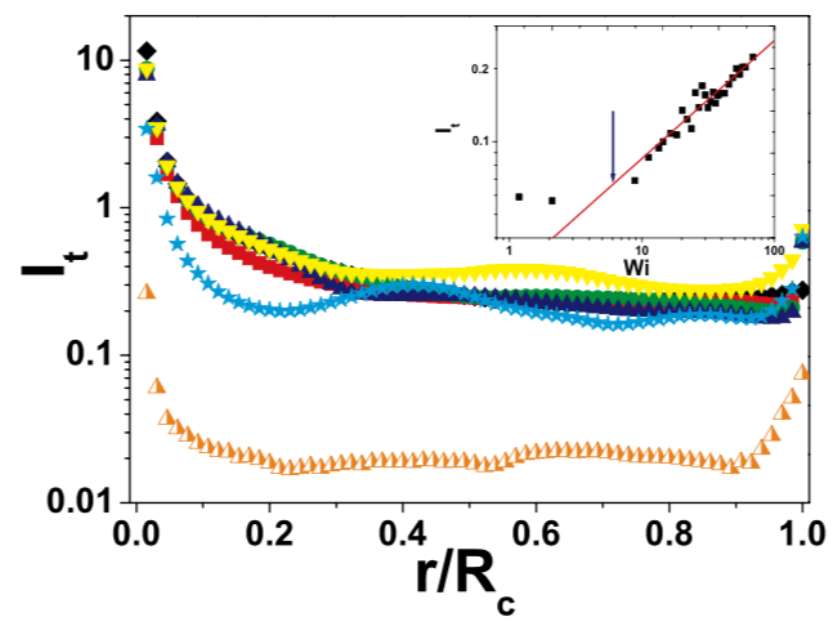

Figure 19. Turbulence intensity versus the reduced radial coordinate at different $W i$ : rhumbs- $W i=31.56$, squares- $W i=27.73$, circles- $W i=23.67$, up triangles- $W i=19.29$, down triangles- $W i=15.48$,stars- $W i=7.54$, half filled triangles-Wi=2.82. The inset shows the dependence of the turbulence intensity on $W i$ at $r / R_{c}=0.85$ obtained from LDV measurements. The full line is a power law fit, $I_{t} \propto W i^{0.49 \pm 0.06}$. The arrow indicates the onset of the elastic instability. The data were collected in setup 2 .

values of the control parameter. However, in contrast to the inertial turbulence, the spectrum of the velocity fluctuations is not related to an energy cascade. A recent theoretical work, Fouxon and Lebedev [2003], suggests the following explanation for the shape of the velocity power spectra displayed in Fig. 21.

In a regime of elastic turbulence, the flow is dominated by a large scale randomly fluctuating velocity field. The passive advection of the stress field by the large scale velocity fields leads to generation of smaller scales stress fields, which are permanently decaying to polymer relaxation. The situation is quite similar to the decay of a passively advected tracer in the Batchelor regime of mixing. The small scales velocity fluctuations are a result of the small scale fluctuations of the stress field. A typical scale at which elastic

without using the Taylor hypothesis. The validity of the TH for elastic turbulent flows will be discussed in section 1.6. 
stresses are pumped can be estimated using the power spectra shown in Fig. 21 ,

$$
L=2 \pi \frac{\int P(k) d k}{\int k \cdot P(k) d k}
$$

Corresponding to fully developed chaotic flow $\operatorname{states}\left(W i>W i_{e t}\right)$, one obtains $L=2.4 \mathrm{~cm}$ for setup 1 and $L=5.9 \mathrm{~cm}$ for setup 2, suggesting that the main energy dissipation takes place at large scale and the physics behind the spectra is similar to the linearly decaying passive scalar problem. Unlike in the case of high $R e$ turbulence, here there is no analogue of the Kolmogorov scale (the apparent flattening of the spectra for large wave numbers is only due to the finite spatial resolution of the PIV measurements) and the spectra should terminate at wave numbers $k_{\text {diff }} \approx 1 / \sqrt{D \cdot \lambda}$ defined by the diffusivity $D$ of the polymer molecules and their relaxation time, $\lambda$. The simultaneous passive advection and relaxation of the elastic stresses results in a fast decay of the stress fluctuations at small scales, which should produce $P(k) \sim k^{-\alpha}$ velocity spectra with $\alpha>3$. The data presented in Fig. 21 displays a decay region $k^{-3.6}$ which agrees rather well with the theoretical prediction, Fouxon and Lebedev [2003], and with previous experimental results ${ }^{8}$, Groisman and Steinberg [2000].

Unfortunately, an experimental technique that allows measurements of the stresses embedded in a fluid flow is not currently available. As suggested in refs. Balkovsky and Fouxon [1999], Balkovsky et al. [2001], the stress generation in the flow of a dilute polymer solution is directly related to the local Lyapunov exponents of the flow (defined by the average logarithmic rate of separation of two initially close Lagrangian trajectories)or, equivalently, to the rms of fluctuations of the velocity gradients.

\footnotetext{
${ }^{8}$ In ref. Groisman and Steinberg [2000] the spatial spectra of the velocity fluctuations have been derived from point velocity measurements by using the Taylor hypothesis. For random flows of a dilute polymer solution in a regime of elastic turbulence, this hypothesis generally fails (see section 1.6). The remarkable agreement between the spectra measured in ref. Groisman and Steinberg [2000] and the directly measured spectra deserves a brief discussion. Following Lumley, Lumley [1965], the relation between the spatial spectra, $P(k)$ and the frequency domain spectra, $P_{1}(f)$ can be written: $P(k)=V \cdot P_{1}(f)-\frac{I_{t}^{2}}{2} \cdot \frac{d^{2}\left(k^{2} \cdot P(k)\right)}{d k^{2}}+O\left(I_{t}^{4}\right)$. If $P(k) \propto k^{\alpha_{2}}$ and $P(f) \propto f^{\alpha_{1}}$, the equation above leads to: $\alpha_{2}-\alpha_{1} \propto \frac{\log \left[1+\frac{I_{t}^{2}}{2} \cdot \alpha_{2} \cdot\left(\alpha_{2}+2\right)\right]}{\log (k)}$. If one plugs in the last equation $\alpha_{2} \approx-3.6$, the difference between the exponents is (for $k \approx 1000 \mathrm{~m}^{-1}$ ) as small as $\alpha_{2}-\alpha_{1} \approx 0.2$.
} 


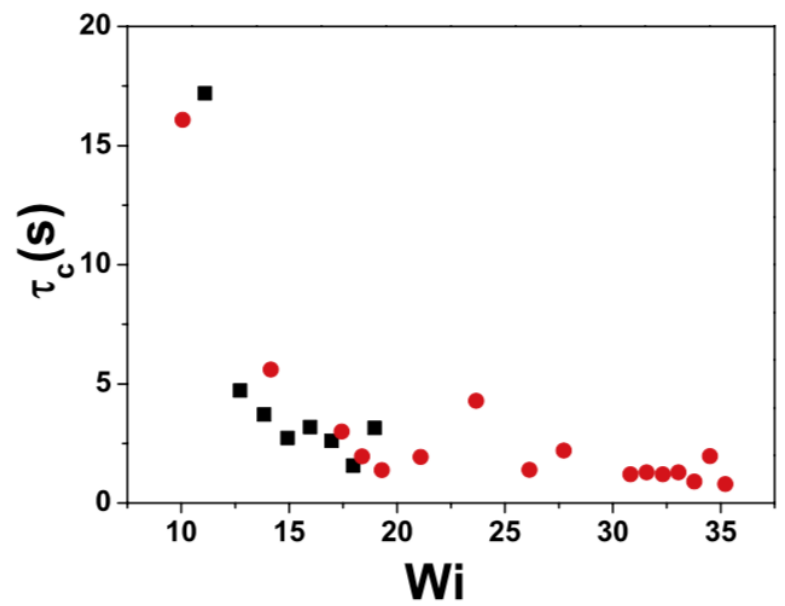

Figure 20. Eulerian correlation times of the tangential velocity component as function of $W i$ : squares-setup 1, circles-setup 2 . 


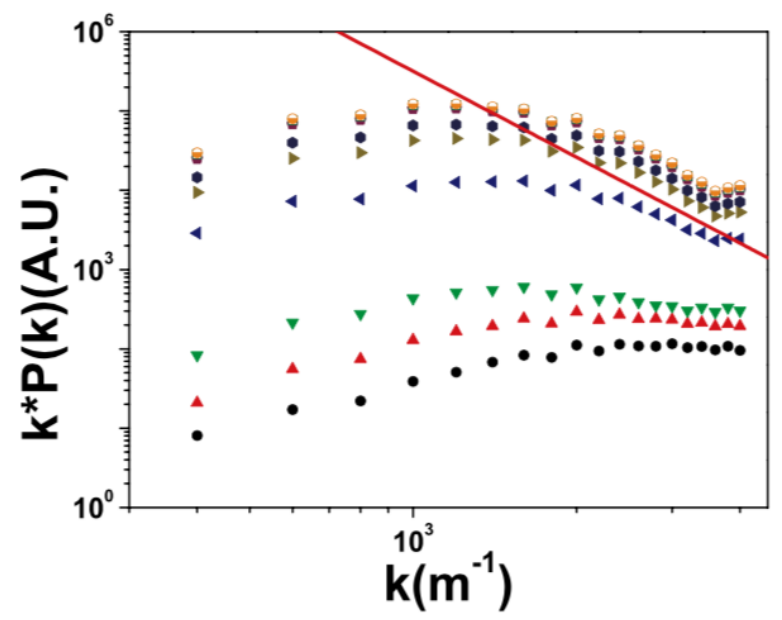

Figure 21. Power spectra of the fluctuations of tangential velocity component at different $W i$ : circles- $W i=4.41$, up triangles- $W i=5.72$, down triangles- $W i=8.32$, left triangles $W i=11.1$, right triangles- $W i=12.7$, hexagons- $W i=13.8$, diamonds- $W i=16$, half filled squares- $W i=18$, empty circles- $W i=19$. Data were collected in setup 1 . 

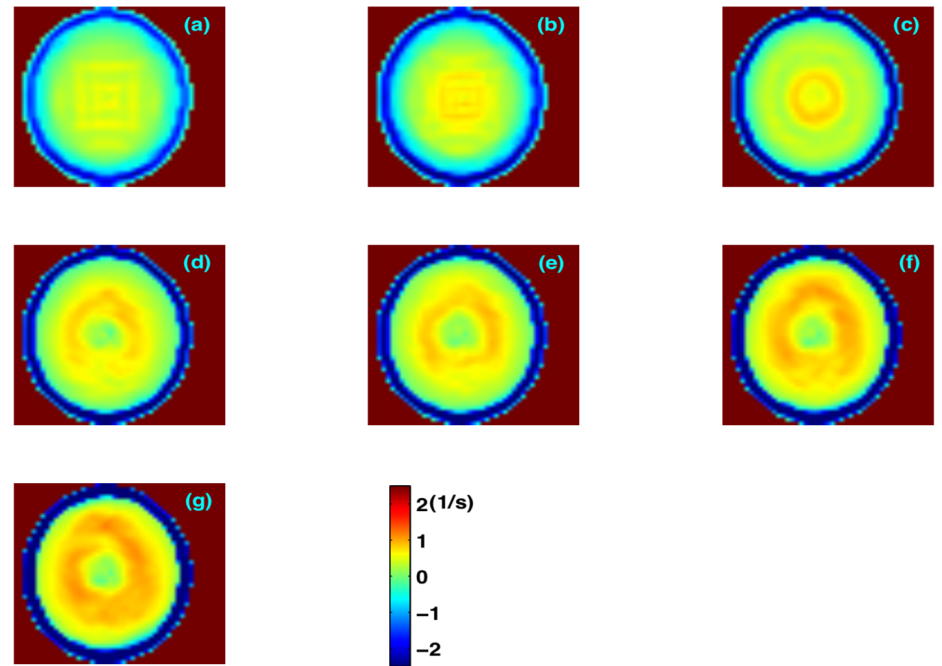

Figure 22. Average gradient of the tangential velocity component in the radial direction, $\frac{\partial v_{\theta}}{\partial r}$, at different $W i$ : (a) $W i=8.32$.(b) $W i=9.88$.(c) $W i=11.1$. (d) $W i=12.72$. (e) $W i=13.83$.(f) $W i=17$ (g) $W i=19$. Data were collected in setup 1 at middle distance between plates. The squared pattern slightly visible in panel (a) is a result of combined peak locking effect and numerical differentiation and should be disregarded. 

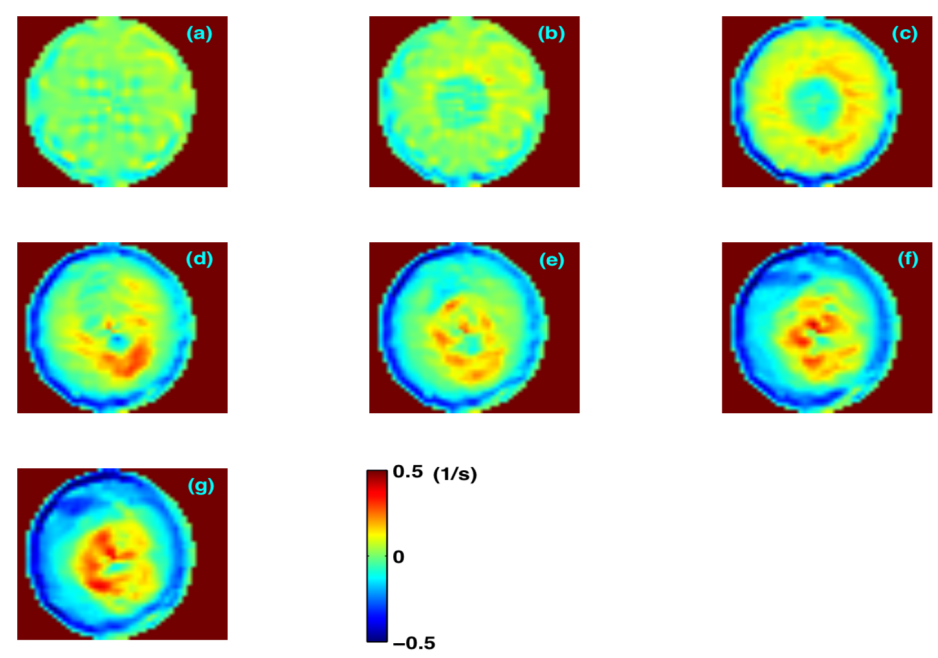

Figure 23. Average gradient of the radial velocity component in the radial direction, $\frac{\partial v_{r}}{\partial r}$, at different $W i$ : (a) $W i=8.32$.(b) $W i=9.88$.(c) $W i=$ 11.1.(d) $W i=12.72$.(e) $W i=13.83$.(f) $W i=17$ (g) $W i=19$. Data were collected in setup 1 at middle distance between plates. 

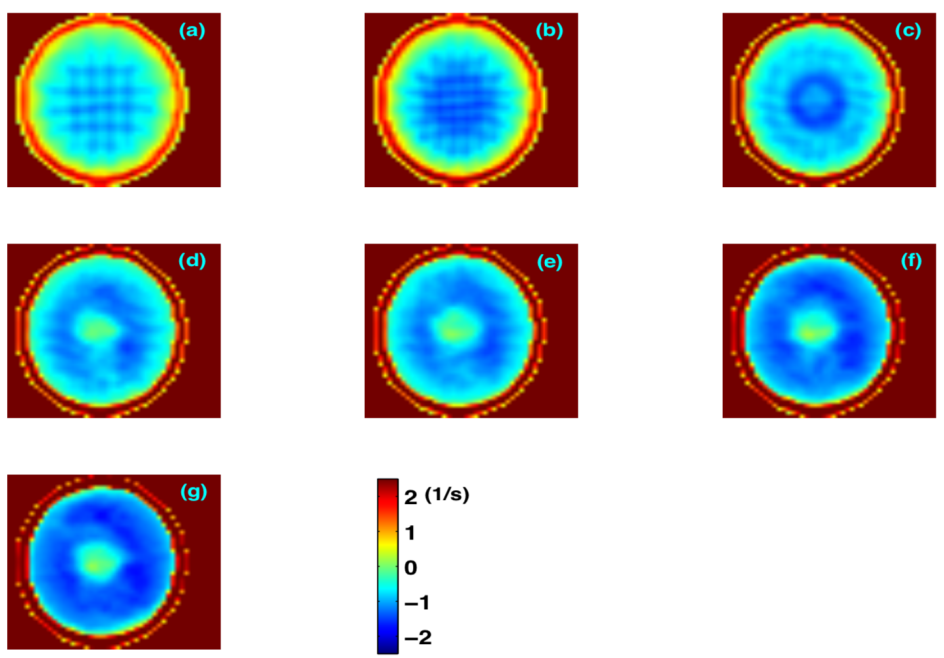

Figure 24. Average vertical component of the vorticity, $\omega_{r \theta}$, at different $W i$ : (a) $W i=8.32$.(b) $W i=9.88$.(c) $W i=11.1$.(d) $W i=12.72 .(\mathbf{e})$ $W i=13.83$.(f) $W i=17$ (g) $W i=19$. Data were collected in setup 1 at middle distance between plates. The squared pattern visible in panels (a), (b) is a result of combined peak locking effect and numerical differentiation and should be disregarded. 

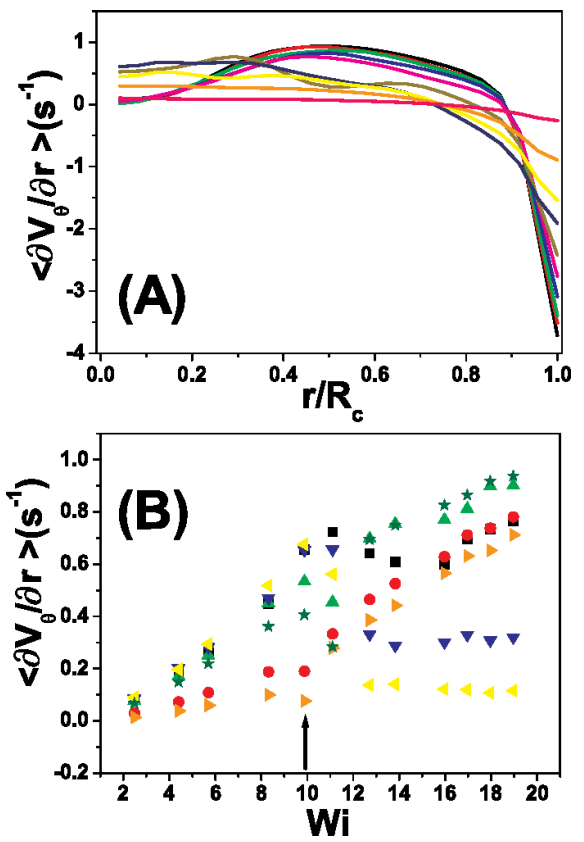

Figure 25. (A) Profiles of the average radial gradient of the tangential velocity at different $W i$ : black- $W i=19$, red- $W i=18$, green- $W i=11$, blue- $W i=16$, magenta- $W i=13.83$, olive- $W i=11.1$, dark blue- $W i=9.88$, yellow- $W i=8.32$, orange- $W i=5.73$, pink- $W i=2.48$. (B) Dependence of the average radial gradient of the tangential velocity on $W i$ at different radial positions: squares- $r / R_{c}=0.3$, circles- $r / R_{c}=0.66$, up triangles$r / R_{c}=0.4$, down triangles- $r / R_{c}=0.2$, left triangles- $r / R_{c}=0.1$, right triangles $-r / R_{c}=0.7$, stars $-r / R_{c}=0.5$. The arrow indicates the onset of the elastic instability. Data were collected in setup 1 at middle distance between plates. 


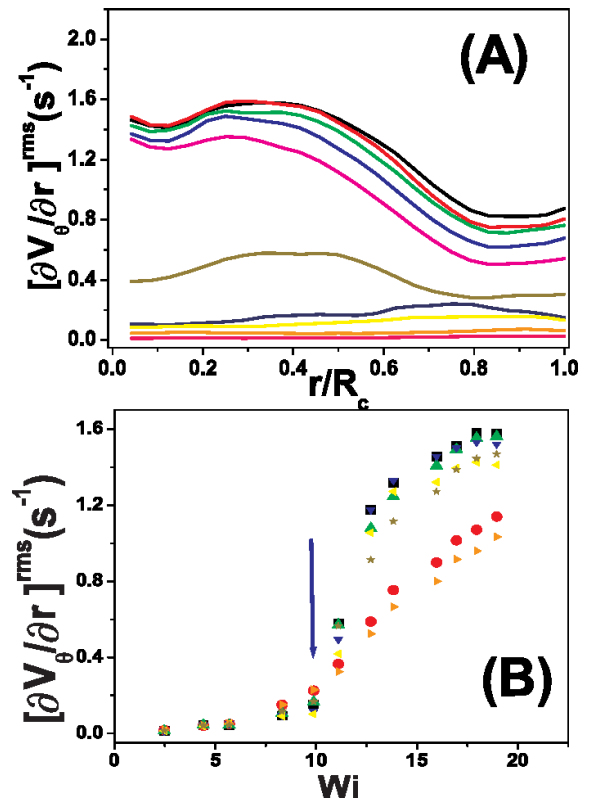

Figure 26. (A) Profiles of the rms of fluctuations of the radial gradient of the tangential velocity at different $W i$ : black- $W i=19$, red- $W i=18$, green- $W i=11$, blue- $W i=16$, magenta- $W i=13.83$, olive- $W i=11.1$, dark blue- $W i=9.88$, yellow- $W i=8.32$, orange- $W i=5.73$, pink- $W i=2.48$. (B) Dependence of the rms of fluctuations of the radial gradient of the tangential velocity on $W i$ at different radial positions: squares- $r / R_{c}=0.3$, circles- $r / R_{c}=0.66$, up triangles $-r / R_{c}=0.4$, down triangles $-r / R_{c}=0.2$, left triangles $-r / R_{c}=0.1$, right triangles- $r / R_{c}=0.7$, stars- $r / R_{c}=0.5$. The arrow indicates the onset of the elastic instability. Data were collected in setup 1 at middle distance between plates. 

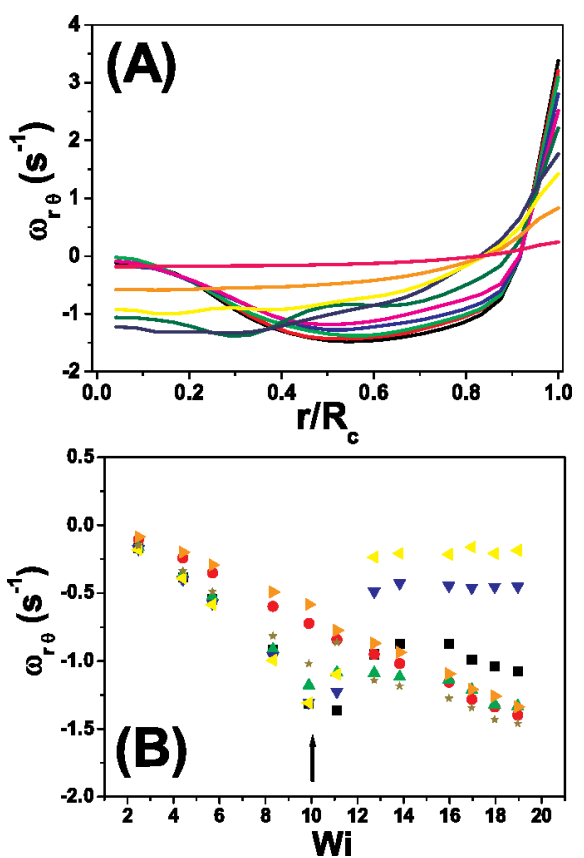

Figure 27. (A) Profiles of the average vorticity, $\left\langle\omega_{r \theta}\right\rangle$, at different $W i$ : black- $W i=19$, red- $W i=18$, green- $W i=11$, blue- $W i=16$, magenta$W i=13.83$, olive- $W i=11.1$, dark blue- $W i=9.88$, yellow- $W i=8.32$, orange- $W i=5.73$, pink- $W i=2.48$. (B) Dependence of the average vorticity on $W i$ at different radial positions: squares- $r / R_{c}=0.3$, circles$r / R_{c}=0.66$, up triangles- $r / R_{c}=0.4$, down triangles- $r / R_{c}=0.2$, left triangles- $r / R_{c}=0.1$, right triangles- $r / R_{c}=0.7$, stars- $r / R_{c}=0.5$. The arrow indicates the onset of the elastic instability. Data were collected in setup 1 . 

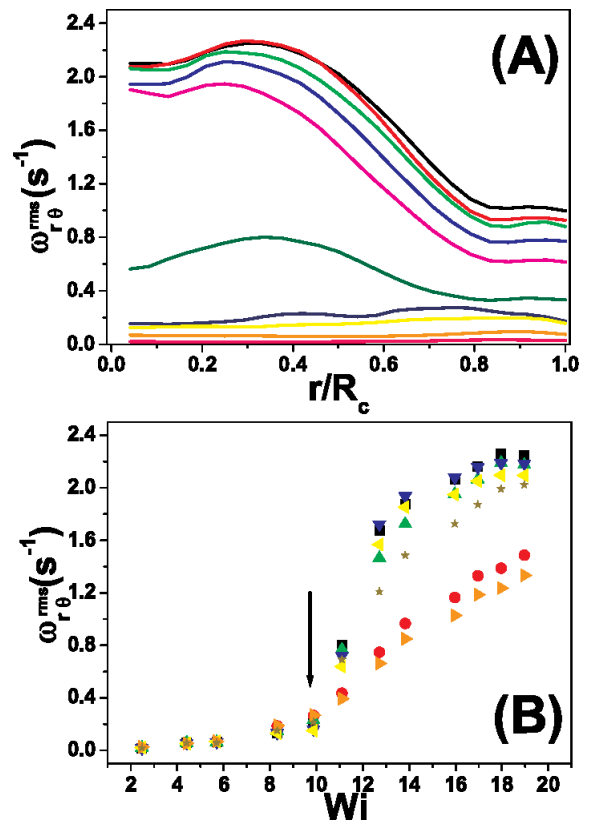

Figure 28. (A) Profiles of the rms of fluctuations of the vorticity, $\omega_{r \theta}^{r m s}$, at different $W i$ : black- $W i=19$, red- $W i=18$, green- $W i=11$, blue- $W i=16$, magenta- $W i=13.83$, olive- $W i=11.1$, dark blue- $W i=9.88$, yellow- $W i=$ 8.32 , orange- $W i=5.73$, pink-Wi=2.48. (B) Dependence of the rms of fluctuations of the vorticity on $W i$ at different radial positions: squares$r / R_{c}=0.3$, circles- $r / R_{c}=0.66$, up triangles- $r / R_{c}=0.4$, down triangles$r / R_{c}=0.2$, left triangles- $r / R_{c}=0.1$, right triangles- $r / R_{c}=0.7$, stars$r / R_{c}=0.5$. The arrow indicates the onset of the elastic instability. Data were collected in setup 1. 

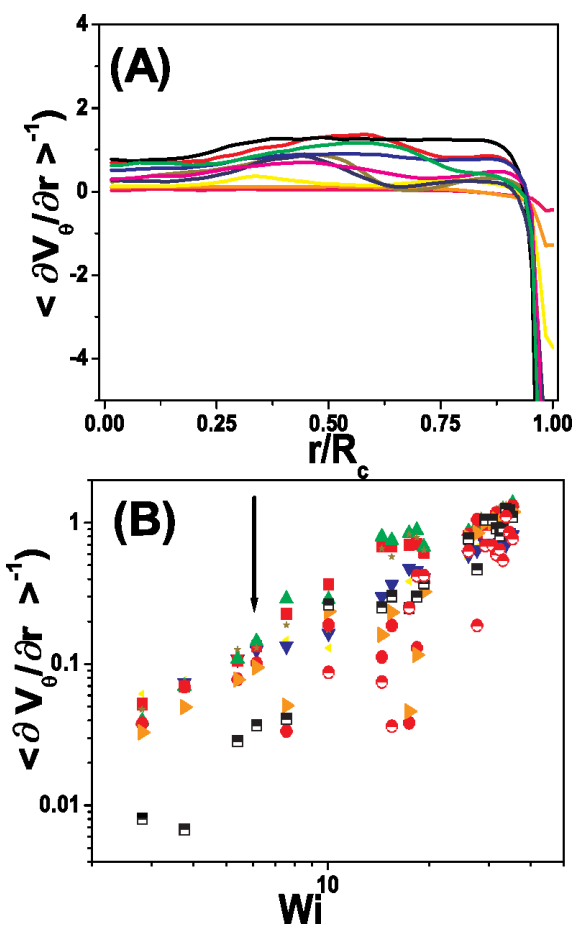

Figure 29. (A) Profiles of the average radial gradient of the tangential velocity at different $W i$ : black- $W i=34.5$, red- $W i=32.3$, green- $W i=27.73$, blue- $W i=26.13$, magenta- $W i=19.29$, dark yellow- $W i=17.43$, dark blue$W i=14.48$, yellow- $W i=10$, orange- $W i=5.4$, pink- $W i=2.82$. (B) Dependence of the average radial gradient of the tangential velocity on $W i$ at different radial positions: squares- $r / R_{c}=0.33$, circles $-r / R_{c}=0.66$, up triangles- $r / R_{c}=0.4$, down triangles- $r / R_{c}=0.2$, left triangles $-r / R_{c}=0.1$, right triangles- $r / R_{c}=0.7$, stars- $r / R_{c}=0.5$, half filled squares- $r / R_{c}=0.8$, half filled circles- $r / R_{c}=0.9$. The arrow indicates the onset of the elastic instability. Data were collected in setup 2. 

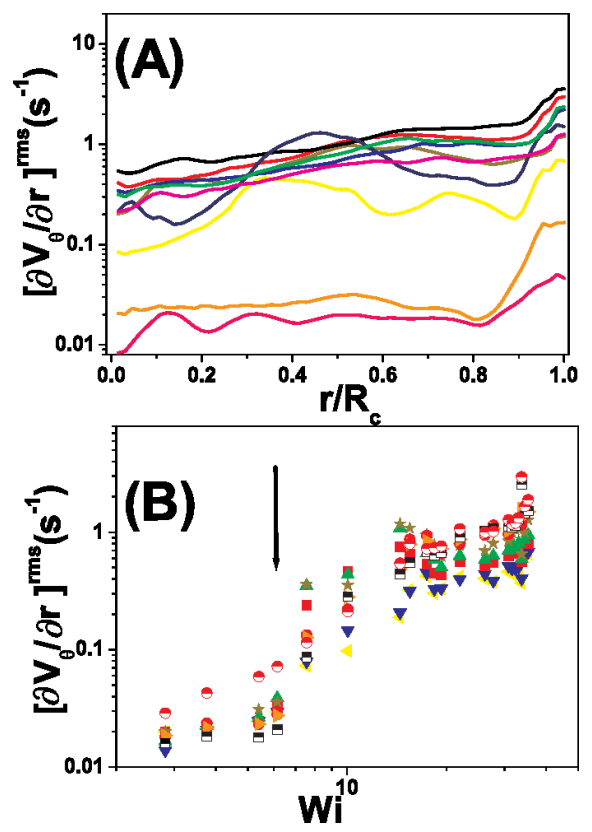

Figure 30. (A) Profiles of the rms of fluctuations of the radial gradient of the tangential velocity at different $W i$ : black- $W i=34.5$, red- $W i=32.3$, green- $W i=27.73$, blue- $W i=26.13$, magenta- $W i=19.29$, dark yellow$W i=17.43$, dark blue- $W i=14.48$, yellow- $W i=10$, orange- $W i=5.4$, pink- $W i=2.82$.(B) Dependence of the rms of fluctuations of the radial gradient of the tangential velocity on $W i$ at different radial positions: squares$r / R_{c}=0.33$, circles- $r / R_{c}=0.66$, up triangles- $r / R_{c}=0.4$, down triangles$r / R_{c}=0.2$, left triangles- $r / R_{c}=0.1$, right triangles- $r / R_{c}=0.7$, stars$r / R_{c}=0.5$, half filled squares- $r / R_{c}=0.8$, half filled circles- $r / R_{c}=0.9$. The arrow indicates the onset of the elastic instability. Data were collected in setup 2 . 


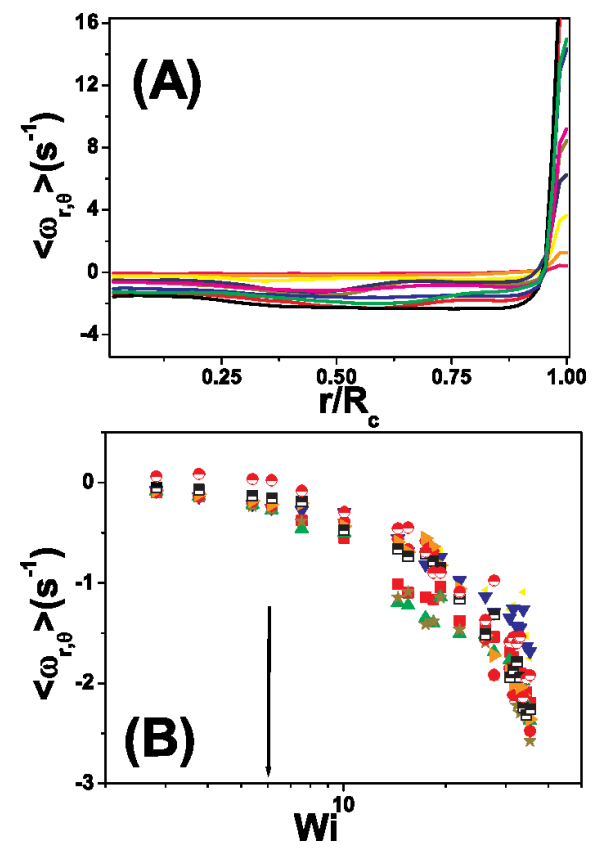

Figure 31. (A)Profiles of the average vorticity, $\left\langle\omega_{r, \theta}\right\rangle$, at different $W i$ : black- $W i=34.5$, red- $W i=32.3$, green- $W i=27.73$, blue- $W i=26.13$, magenta- $W i=19.29$, dark yellow- $W i=17.43$, dark blue- $W i=14.48$, yellow- $W i=10$, orange- $W i=5.4$, pink- $W i=2.82$ (B) Dependence of the average vorticity on $W i$ at different radial positions: squares- $r / R_{c}=0.33$, circles- $r / R_{c}=0.66$, up triangles $-r / R_{c}=0.4$, down triangles $-r / R_{c}=0.2$, left triangles- $r / R_{c}=0.1$, right triangles- $r / R_{c}=0.7$, stars- $r / R_{c}=0.5$, half filled squares- $r / R_{c}=0.8$, half filled circles- $r / R_{c}=0.9$. The arrow indicates the onset of the elastic instability. Data were collected in setup 2.

The measurements of time dependent flow fields allowed us to calculate directly ${ }^{9}$ the average gradients and the rms of their fluctuations of each velocity component in both polar directions. Typical spatial distributions of the time averaged gradient of the tangential velocity component in the radial direction, $\frac{\partial v_{\theta}}{\partial r}$, and of the time averaged radial velocity component in the radial direction, $\frac{\partial v_{r}}{\partial r}$ are displayed in Figs. 22 and 23 for several values

\footnotetext{
${ }^{9}$ Again, without using the Taylor hypothesis.
} 

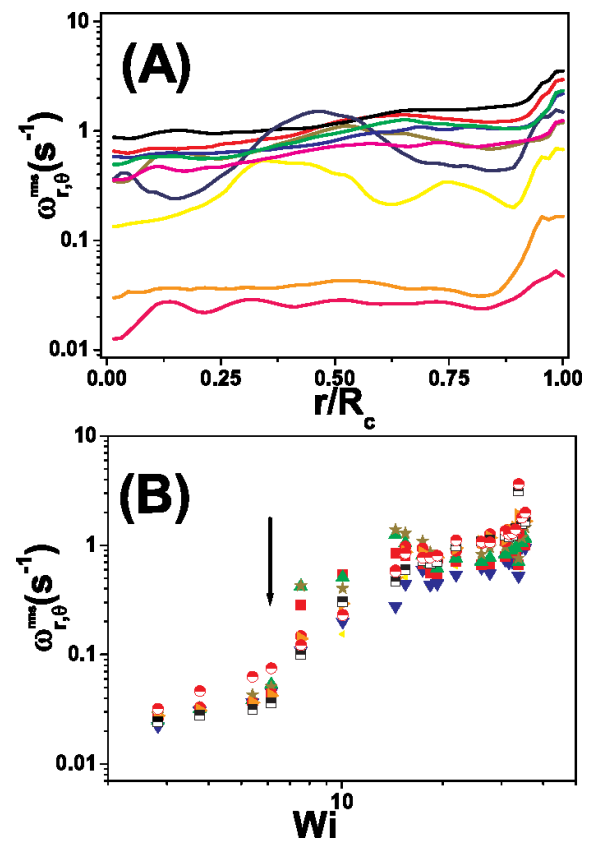

Figure 32. (A) Profiles of the rms of fluctuations of the vorticity, $\omega_{r, \theta}^{r m s}$, at different $W i$ : black- $W i=34.5$, red- $W i=32.3$, green- $W i=27.73$, blue- $W i=26.13$, magenta- $W i=19.29$, dark yellow- $W i=17.43$, dark blue- $W i=14.48$, yellow- $W i=10$, orange- $W i=5.4$, pink- $W i=2.82$.(B) Dependence of the rms of fluctuations of the vorticity on $W i$ at different radial positions: squares- $r / R_{c}=0.33$, circles- $r / R_{c}=0.66$, up triangles$r / R_{c}=0.4$, down triangles- $r / R_{c}=0.2$, left triangles- $r / R_{c}=0.1$, right triangles- $r / R_{c}=0.7$, stars- $r / R_{c}=0.5$, half filled squares- $r / R_{c}=0.8$, half filled circles- $r / R_{c}=0.9$. The arrow indicates the onset of the elastic instability. Data were collected in setup 2 . 
of the control parameter (the data refers to setup 1).

Spatial distributions of the time averaged vorticity are presented in Fig. 24 for several values of $W i$. As one can see in Fig.22(b), below the onset of the elastic instability, $W i<W i_{c}$, the mean gradient of the tangential component is rather constant in the bulk (consistent with a rigid body rotation, that is a linear increase of the tangential velocity with the radial coordinate, Fig. 14 ) and sharply decreasing towards negative values in the boundary. In the intermediate flow region, $W i_{c}<W i<W i_{e t}$, the spatial distribution of the mean gradient has an apparent ring shaped topology (Fig. $22(\mathrm{c})$ ) which is consistent with the large scale toroidal vortex developed in this region. The large values of the velocity gradient in the boundary explain why, once the hoop stresses destabilise the flow the instability front propagates from the boundaries to the bulk as the control parameter is increased (Fig. 18). A further increase of the control parameter in the region $W i>W i_{e t}$ results in a rather uniform distribution of the gradients in the bulk and large negative values in the boundary. Radial profiles of the mean gradient of the tangential velocity component in the radial direction displayed in Fig. 25(A) reveal a rather uniform distribution of the gradients in the bulk of the flow and a sharp increase near the boundary. The typical dependencies on the control parameter are displayed in Fig. 25(B).

As one can see in Figs.26 30, $\left(\frac{\partial v_{\theta}}{\partial r}\right)^{r m s}$ saturates at a value $\approx 1.4 s^{-1}$ which is of the same order of magnitude with the inverse Eulerian correlation time (see Fig. 20) and the inverse polymer relaxation time. Profiles of the average vorticity and its dependence on the control parameter are shown in Fig. 27,1.24. In Figs. 28, 32 are shown profiles of the rms of fluctuations (panel (A)) of the vorticity and their $W i$ dependence (panel (B)). In both of the setups tested a similar behaviour of the gradients and the rms of their fluctuations (though the increase in the boundary looks less pronounced) has been observed.

The saturation of the rms of fluctuations of the velocity gradients in the region $W i>W i_{e t}$ at values comparable to the inverse polymer relaxation time is consistent with the theoretical predictions corresponding to the case when the back reaction of the polymer molecules to the flow is switched on. A theoretical explanation for this experimentally observed fact will be given in Sec. 5.

\subsection{Lagrangian frame dynamics in a regime of Elastic Tuerbu- lence}

The discovery of a deterministic chaos has substantially changed the classical view point on the origin of randomness in a fluid flow and pro- 
vided a considerable number of powerful tools in understanding bifurcation phenomena in hydrodynamic systems Brandstäter et al. [1983]. One of the quantitative measures of the degree of randomness of a fluid flow is the largest Lyapunov exponent (LLE), which defines the average rate of an exponential separation of two neighbouring trajectories:

$$
L L E=\lim _{t \rightarrow \infty} \lim _{\delta(0) \rightarrow 0} \frac{1}{t} \log \left(\frac{\delta(t)}{\delta(0)}\right)
$$

where $\delta(t)=\left\|x_{2}(t)-x_{1}(t)\right\|$ is the Euclidian distance between two trajectories at time $t$. The LLE is the main flow characteristics that determine both the local dynamics and conformations of the polymer molecules Balkovsky and Fouxon [1999], Balkovsky et al. [2001], Gerashchenko, S. et al. [2005]. Thus, the knowledge of the LLE together with the knowledge of the single molecule polymer relaxation time $\tau_{r}$ defines the criterion for the coil-stretch transition Balkovsky and Fouxon [1999], Balkovsky et al. [2001], Gerashchenko, S. et al. [2005]: $\lambda \cdot \tau_{r}=1$. In most of the previous experimental works, the LLE was measured by reconstruction of the phase portrait from measurements of a single point observable. Although from technical point of view this approach might look less demanding than a direct measurement of the particle pair separations, there are few rather sensitive issues that should be carefully addressed: a proper choice of the time delays and the embedding dimension, length and noise level of the data sets. We are aware of only one experimental work that deals with direct measurements of the Lagrangian trajectories and the statistics of pair separations Boffetta, G. et al. [1999]. The main purpose of this Lagrangian frame investigation is to understand the relation between LLE's and the relevant Eulerian time scales of the flow: velocity correlation time, inverse velocity gradients, and the magnitude of their fluctuations. In experimental and numerical simulations the infinite time limit required by Eq. (8) is unattainable. Besides, due to the finite size of experimental setups the dispersion of initially closely located particles cannot reach a truly asymptotic regime due to interaction with boundaries. A way to overcome these difficulties is to use the finite time Lyapunov exponents approach (FTLE) Boffetta et al. [2002], Lacorata et al. [2001], Artale et al. [1997], Boffetta et al. [2003], that is to look on the average rate of separation of initially close particles during finite times. This approach is particularly suitable in the case, when the characteristic scale of the velocity field, $l_{u}$, is comparable with the system size, L, and it has been recently employed in experiments and numerical simulations Gerashchenko, S. et al. [2005], Boffetta et al. [2002], Artale et al. [1997]. The FTLE is defined by 


$$
\gamma(t)=\frac{1}{t}\left\langle\log \frac{\delta(t)}{\delta(0)}\right\rangle_{e}
$$

where the ensemble average is taken over all particle pairs initially contained in clusters of radius $\delta(0) \ll l_{u}$. For sufficiently small initial particle separation, $\delta(0)$, the FTLE becomes a rather good estimate of the LLE Boffetta et al. [2002], Artale et al. [1997].

We conducted our measurements above the elastic instability threshold at $(W i>6)$ and higher Groisman and Steinberg [2000], Larson [1992], where the chaotic flow results from the elastic stresses only since inertial contribution is low $(R e<16)$. The velocity field measurements presented in the previuos section show that, above the onset of the elastic instability the swirling flow is dominated by a randomly fluctuating large scale vortex. In contrast to the case of inertial turbulence, where the flow can be considered smooth only below the Kolmogorov scale, the elastic turbulent flow is smooth at all scales, and no analogue of the dissipation scale exists. The large scale of the velocity field, on which energy pumping takes place, is defined by the elastic instability and is about the system size, i.e. $l_{u} \approx R_{d}$ . Thus, the flow smoothness over a broad range of scales fully justifies the use of the FTLE approach. Numerical Lagrangian trajectories were obtained by integrating in time $\frac{d \vec{x}}{d t}=\vec{v}(\vec{x}, t)$ with a 5 th order adaptive step Runge-Kutta integrator (Shampine and Watts program based on Fehlberg's Runge-Kutta pair of order 4 and 5). Instantaneous velocity at a tracer position was obtained by a trilinear space-time interpolation of the velocity field measured by PIV at regularly gridded space-time points. The time step of the interpolation was $40 \mathrm{~ms}$. Fig. 33 shows the initial distribution of the numerical tracers (panel (a)) and after $1 s$ (panel (b)).

Statistics of particle pair separations The statistics of particle pair separations was carried out for all the particle pairs initially distributed (uniformly) inside clusters of radius $R=0.1 R_{c}$ during 0.4 seconds (this total integration time was smaller then a half turnover time in the whole range of angular velocities). In order to collect statistically sufficient separation data, this procedure was repeated 200 times until the whole velocity field time series has been used. However, a major concern was whether the average length of the numerical trajectories is consistent with that of real particle trajectories. Thus, in order to check the correctness and consistency of our approach, we have alternatively used particle tracking velocimetry (PTV) technique to measure the statistics of real particle pair separations. Direct measurements of particle trajectories have shown that the average persistence time of real particles in the observation plane was consistent 


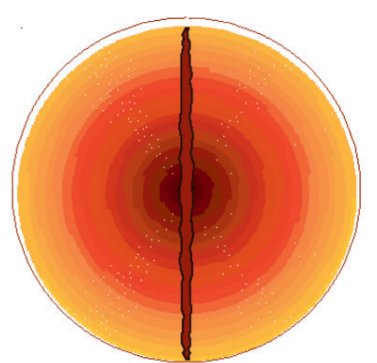

(a)

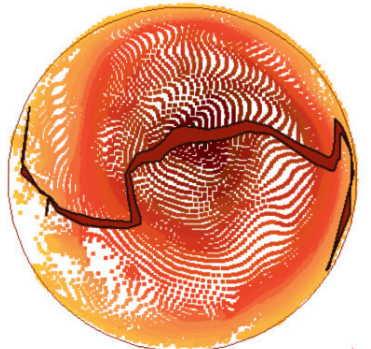

(b)

Figure 33. Numerical tracers at $t=0$ (a) and $t=1 \mathrm{~s}$ (b) for $W i=33$. The false color maps the radial coordinate.

with the average length of the numerical trajectories. This additional check is fully justified in our case because above the onset of the primary elastic instability, the flow is three-dimensional, and tracking numerical tracers for too long times would be physically meaningless.

As shown in the previous section, the Eulerian correlation time is $\tau_{c} \approx$ $1 s$ (Fig.20), which is about 2.5 times larger then the integration time of the numerical trajectories and about 240 times smaller than the total data acquisition time. For each angular velocity investigated, we have defined FTLE by the position of the peak of the probability distribution function (PDF), $P(\gamma, t)$ of the separation rates. In Fig.34 we show the Cramer rate functions defined by

$$
S(\gamma)=-\frac{1}{t} \cdot \log \left(\frac{P(\gamma, t)}{P_{\max }}\right)
$$

where $P_{\max }$ is the maximum of the PDFs.

The FTLEs as function of Wi are shown in Fig.35. Although the scale dependence of the mean separation rates plotted in the inset of Fig.35 does not show the expected decay corresponding to the mean turnover time Boffetta, G. et al. [1999], its flatness in the range of separation times considered is rather encouraging and suggesting that FTLE approach works rather robustly for our random flow. The FTLE dependence on $W i$ exhibits a sudden jump corresponding to the first flow reorganisation in the intermediate regime, followed by a slower increase in the fully developed elastic turbulent states.

Comparing the values of FTLE (Fig.35) with the Eulerian correlation 


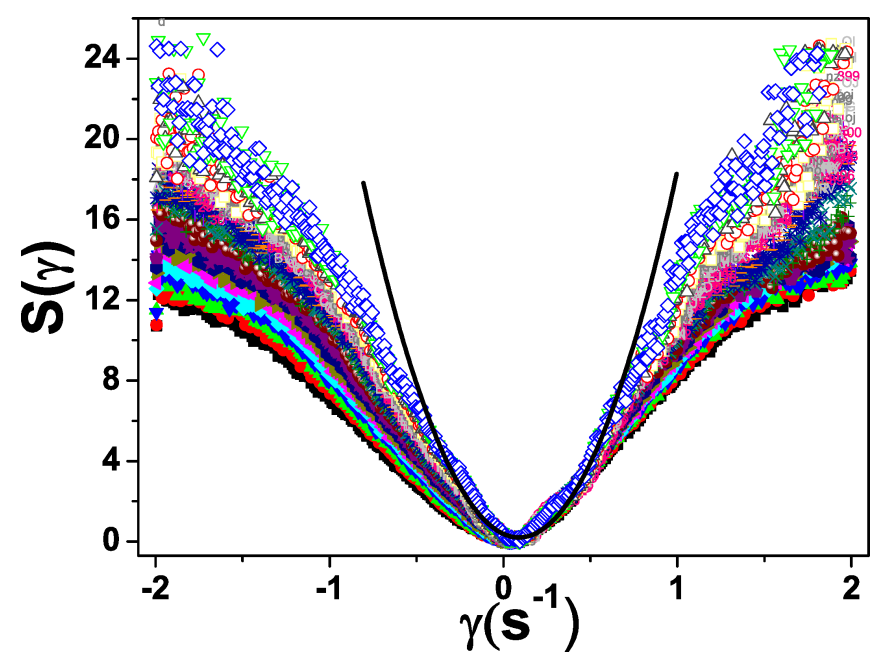

Figure 34. Cramer functions of particle pair separation rates for 25 different separation times (ranging from 0.16 to $2.08 s$ ) at $W i=34$. The full line is the parabolic fit.

times (Fig.32) one notices that in fully developed random flow the following relation holds: $\gamma_{a v} \cdot \tau_{c} \approx 0.1$. This discrepancy with the suggestion made in Balkovsky and Fouxon [1999] is probably due to the long range correlations 10 existing in the flow and to insufficient time statistics (which is one of the limitations of image based flow investigation techniques, such as PIV and PTV).

Comparison of FTLEs with rms of the velocity gradients (Fig. 30) led to the conclusion that

$$
\left(\frac{\partial v_{\theta}}{\partial r}\right)^{r m s} \approx 7 \cdot \gamma_{a v}
$$

\section{Lagrangian frame flow intermittency}

The FTLEs are the first moments of PDF, and they give no information about the degree of intermittency. Equation (8) defines the average rate of a separation of nearby trajectories but does not provide any information

\footnotetext{
${ }^{10}$ The measurements of the time space cross correlation function presented in the next section support this point.
} 


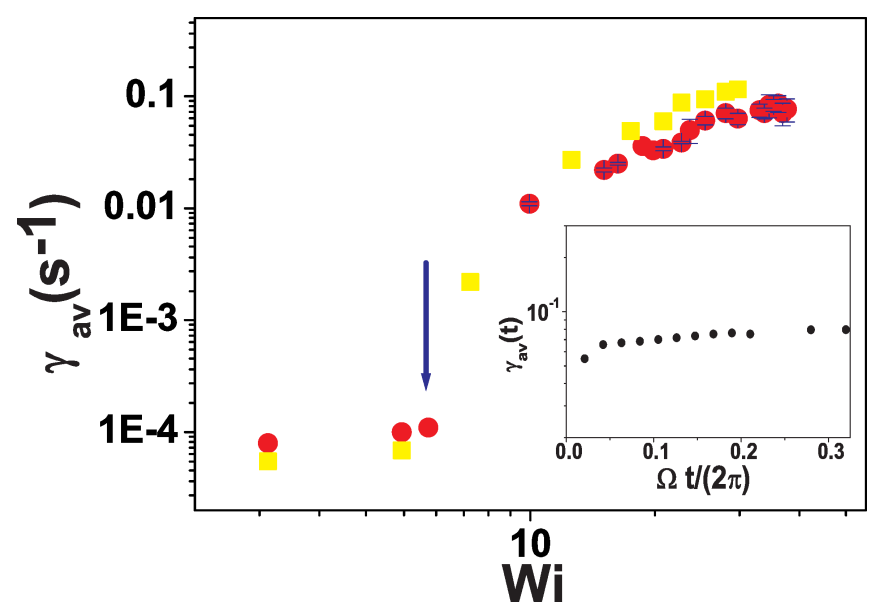

Figure 35. Finite time Lyapunov exponents defined by the location of the minima of the Cramer rate functions of the particle pairs separations versus Wi: squares, from PTV approach, circles from PIV based approach. The arrow indicates the onset of the primary elastic instability. The inset shows the dependence of FTLE on the separation time; the horizontal axis is normalised by the period of rotation of the disk.

about the fluctuations around this average. In order to characterize the Lagrangian flow intermittency one has to reconstruct PDF of the particle pair separations by calculating the generalized Lyapunov exponents (GLE), which are related to the high order moments of the statistics of the particle pair separations Paladin and Vulpiani [1987]. The GLE of order q is defined by

$$
L(q)=\lim _{t \rightarrow \infty} \lim _{\delta(0) \rightarrow 0} \frac{1}{t} \log \left(\left|\frac{\delta(t)}{\delta(0)}\right|^{q}\right)
$$

In the infinite time limit, when the Central Limit Theorem is expected to work, it has been shown Paladin and Vulpiani [1987] that for small values of $q$ :

$$
L(q)=\lambda \cdot q+\frac{\mu}{2} \cdot q^{2}
$$

where the second cumulant is defined as 


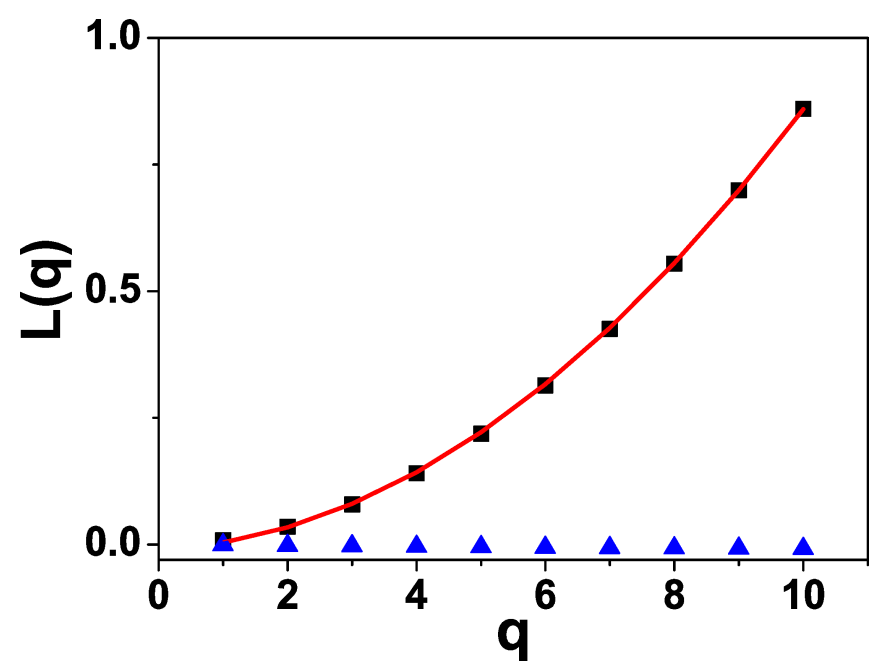

Figure 36. Generalised Lyapunov exponents as a function of the order $q$ of the moments at different $W i$ : squares- $W i=33$, triangles- $W i=2.82$. The full line is the parabolic fit, which gives $\mu=0.0167 \pm 0.00005$.

$$
\mu=\lim _{t \rightarrow \infty} \lim _{\delta(0) \rightarrow 0} \frac{1}{t}\left[\left\langle\log \left|\frac{\delta(t)}{\delta(0)}\right|^{2}\right\rangle_{e}-(\lambda \cdot t)^{2}\right]
$$

In Fig.36 we show as a GLE as a function of the order q of the moments. According to Paladin and Vulpiani [1987] the onset of intermittent behavior corresponds to $\frac{\mu}{\gamma_{a v}} \approx 1$ that is well above of the value obtained in the experiment. Although the parabolic dependence of the generalised Lyapunov exponents agrees well with theory Paladin and Vulpiani [1987], the second cumulants obtained from the fit (Fig.36) display a rather big scatter being plotted versus the angular velocity of the upper plate. This is probably related to the power law propagation of the instrumental errors in the calculation of $L(q)$. Alternatively, one can characterize the intermittency by measuring the deviation of PDFs from a Gaussian shape (as illustrated in Fig.34, around $\gamma_{a v}$ the Cramer function shows a parabolic behavior). Recalling the scaling of the even order moments of the Gaussian PDF with the variance, one should look at the normalized moments:

$$
\overline{M_{2 k}}=M_{2 k} \cdot \frac{2^{k} \cdot k !}{(2 k) !}
$$




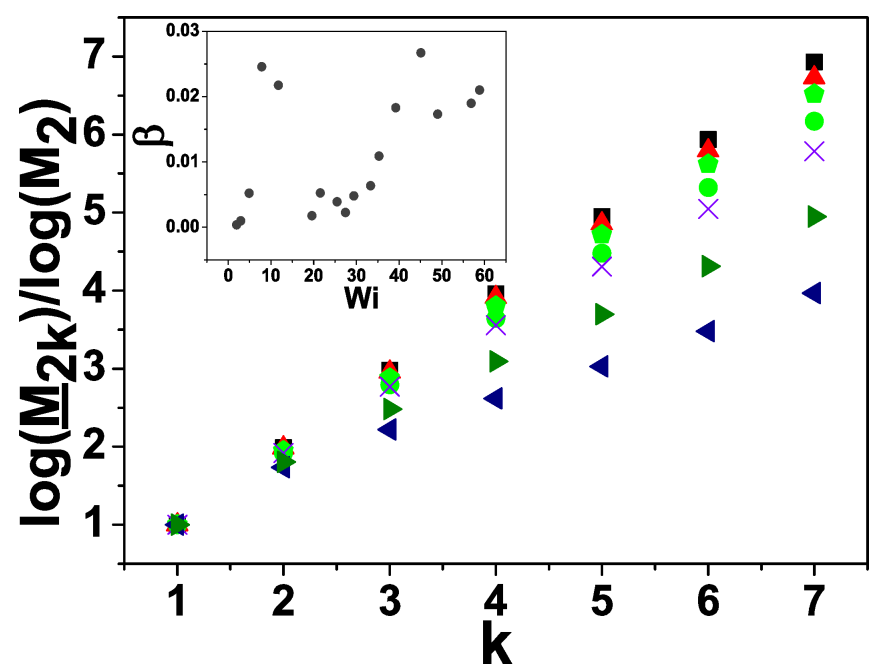

Figure 37. Normalized (see text) high order moments at different $W i$ : squares, - $W i=1.9$, up triangles- $W i=4.8$, diamonds $-W i=29$, circles$W i=33.3$, crosses- $W i=38$, right triangles- $W i=56$, left triangles- $W i=$ 58. The inset shows an estimate of the degree of the flow intermittency versus $W i$.

The deviation from the Gaussian shape can be quantified by the parameter defined as

$$
\frac{\log \left(\overline{M_{2 k}}\right)}{\log \left(M_{2}\right)}=k-\beta \cdot k^{2}
$$

The dependence of the right hand side of Eq. (16) on the order of the moment is shown in Fig. 37.

As the angular velocity of the upper plate increases, the flow intermittency exhibits a sudden jump corresponding to the primary elastic instability followed by a second discontinuity corresponding to the second flow reorganisation (see inset in Fig.37). As one can see from the plot in Fig.37 at sufficiently large angular velocities the deviation from a Gaussian linear dependence is rather significant indicating a flow intermittency. 


\subsection{Applicability of the Taylor hypothesis in a regime of Elastic Turbulence}

More than six decades ago, Taylor introduced a conjecture, by which the spatial structure of a turbulent flow field can be inferred only from single point measurements of its fluctuations Taylor [1938]. This assumption, known as Taylor frozen flow hypothesis (TH), has become a main tool in the experimental studies on turbulence particularly in situations, where full flow field measurements were unavailable Lesieur [2008]. The validity of the TH is mainly conditioned by the existence of a large mean flow comparative to the fluctuations, $\frac{v^{\prime}}{V} \ll 1$. Here $v^{\prime}$ stands for the fluctuating part of the instantaneous velocity, and $V$ for the mean flow velocity. Secondly, the frozen flow assumption works well only for structures, which are much smaller than the typical scale of the mean flow Lin [1953]. Although few theoretical works do exist on the subject Lin [1953], Lumley [1965], a general theoretical frame work is still lacking and thus, the question of validity of the TH should be addressed experimentally in each particular case. On the other hand, there exists a large body of experimental works on this subject mainly related to 3D inertial turbulent flows Dahm and Southerland [1997], Quadrio and Luchini [2003], Mi and Antonia [1994] and more recently for a 2D turbulent soap film flow Belmonte et al. [2000].

In this section we address the problem of validity of $\mathrm{TH}$ in a random in time and spatially smooth velocity field. Such type of a chaotic flow is realised, e.g., in hydrodynamic turbulence at scales below the Kolmogorov length Lesieur [2008], in 2D turbulenceJullien et al. [2000], Amarouchene and Kellay [2004].

As shown Sec. Sec. 4.3, the flow of a dilute polymer solution above the onset of the elastic instability is an ideal realisation of both randomness (in time) and spatial smoothness.

We conducted our measurements in setup 2, above the onset of the elastic instability Muller et al. [1989b] at $(W i>6)$ and in the region of the elastic turbulence Groisman and Steinberg [2000]. The velocity field measurements presented in the previous section show that, above the onset of the elastic instability the flow is dominated by a randomly fluctuating large scale vortex Groisman and Steinberg [2000]. In contrast to the case of inertial turbulence, where the space velocity correlations decay rather fast over distances larger than the Kolmogorov scale, the elastic turbulent flow is smooth and strongly correlated over space on a size of the fluid container.

In most of the previous experimental studies of the validity of the $\mathrm{TH}$, single point velocity time traces were measured simultaneously at different points displaced along the mean flow direction and the validity of the $\mathrm{TH}$ was tested either by analysing the velocity correlations downstream, or by 


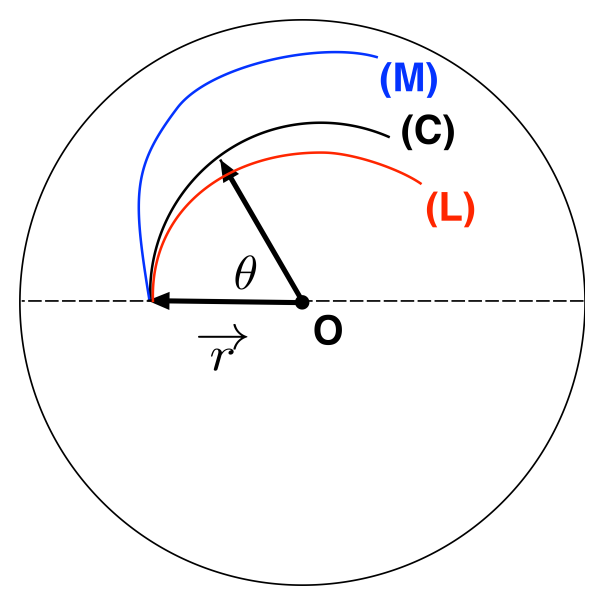

Figure 38. Schematic view of the flow geometry: (M)- mean flow line, (L) - measured Lagrangian trajectory (C) - circular arc.

comparing the velocity time derivatives with the velocity gradients. From the point of view of robustness, these two approaches are complementary: the first is suitable for the case, when velocity is well resolved in space but not well resolved in time, whereas the second requires a high resolution in time but is not strongly limited by the space resolution.

As already discussed in Sec. 4.3, a characteristic scale of a system, at which energy pumping takes place, can be estimated from the space power spectrum of the velocity fluctuations (Fig. 21(b)) as

$$
L=2 \pi \frac{\int P(k) d k}{\int k \cdot P(k) d k}
$$

Then we have obtained for $W i=70 \approx 5.9 \mathrm{~cm}$, which is comparable to the size of the setup 2. As one can see from Fig. 19 which displays the turbulence intensity, $I_{t}=\sqrt{\left\langle v^{\prime 2}\right\rangle_{t}} / V$, versus the reduced radial coordinate in setup 2, the central flow region is characterised by large fluctuations and vanishingly small mean flow whereas in the off central region the the level of fluctuations decreases to about $25 \%$ of the mean flow.

Fig. 39 displays velocity time series at three different locations along the mean flow line. As one can see from panels (a) and (b), for sufficiently small separations, $s$, the two velocity time series are almost identical, up to 
a shift in time. For larger separations [panel (c)] the velocity fluctuations are less correlated, and the two time series display clear differences.

Shape of the cross-correlation functions. In order to check whether the velocity fluctuations are transported without evolving dynamically (the flow is frozen), we measure the cross-correlation of the velocity fluctuations between neighboring points separated by the distance $s, C_{i j}(\tau, \overrightarrow{\Delta r}, \vec{r})$ defined by:

$$
C_{i j}(\tau, \overrightarrow{\Delta r}, \vec{r})=\frac{\left\langle v_{i}(\vec{r}, t) \cdot v_{j}(\vec{r}+\overrightarrow{\Delta r}, t-\tau)\right\rangle_{t}}{v_{i 1}^{r m s} \cdot v_{j 2}^{r m s}}
$$

where $v_{1,2}^{r m s}$ is the rms of velocity at the points $\vec{r}, \vec{r}+\overrightarrow{\Delta r}$ and $i, j=r, \theta$ are the polar coordinates (Fig. 38). It is worth noting that, since the mean flow is curvilinear, $C_{i j}$ depends not only on the magnitude of the displacement between neighboring points, $|\overrightarrow{\Delta r}|$, but also on the path along which the displacement is considered and its length $s$. Thus, in order to check the sensitivity of our results on the choice of the displacement path, we have alternatively considered displacements along circular arcs (around the center of the setup), time average flow lines, and Lagrangian trajectories. Secondly, because the field of fluctuations is strongly non homogenous (Figs. 29,30), the dependence on the initial position $\vec{r}$ should also be considered. For the simplicity of the notation, we will further refer the cross-correlation functions as $C_{i j}(\tau, s, \vec{r})$.

Let us now focus on the relation between flow smoothness $\left(\frac{\partial^{k} \vec{v}(\vec{r}, t)}{\partial \vec{r}^{k}} \approx\right.$ $0, k \geq 3$.) and the shape of the cross-correlations. Within the approximation of vanishing high order spatial derivatives and for small displacements, $s$, and delay times, $\tau$, one can easily decouple the space and time dependencies in the definition of the cross-correlation function [Eq. (18)]:

$$
\begin{array}{r}
C_{i j}(\tau, s, \vec{r}) \simeq \frac{\left\langle v_{i}(\vec{r}, t) \cdot v_{j}(\vec{r}, t)\right\rangle_{t}}{v_{i 1}^{r m s} \cdot v_{j 2}^{r m s}}+s \cdot \frac{\left\langle v_{i}(\vec{r}, t) \cdot \frac{\partial v_{j}(\vec{r}, t)}{\partial r}\right\rangle_{t}}{v_{i 1}^{r m s} \cdot v_{j 2}^{r m s}}- \\
\tau \cdot \frac{\left\langle v_{i}(\vec{r}, t) \cdot \frac{\partial v_{j}(\vec{r}, t)}{\partial t}\right\rangle_{t}}{v_{i 1}^{r m s} \cdot v_{j 2}^{r m s}}-s \cdot \tau \cdot \frac{\left\langle v_{i}(\vec{r}, t) \cdot \frac{\partial^{2} v_{j}(\vec{r}, t)}{\partial r \partial t}\right\rangle_{t}}{v_{i 1}^{r m s} \cdot v_{j 2}^{r m s}}+\frac{\tau^{2}}{2 !} \cdot \frac{\left\langle v_{i}(\vec{r}, t) \cdot \frac{\partial^{2} v_{j}\left(\vec{r}_{, t}\right)}{\partial t^{2}}\right\rangle_{t}}{v_{i 1}^{r m s} \cdot v_{j 2}^{r m s}}+ \\
\frac{s^{2}}{2 !} \cdot \frac{\left\langle v_{i}(\vec{r}, t) \cdot \frac{\partial^{2} v_{j}\left(\vec{r}^{2}, t\right)}{\partial r^{2}}\right\rangle_{t}}{v_{i 1}^{r m s} \cdot v_{j 2}^{r m s}}
\end{array}
$$

A typical space time cross-correlation surface is shown in Fig. 40. 


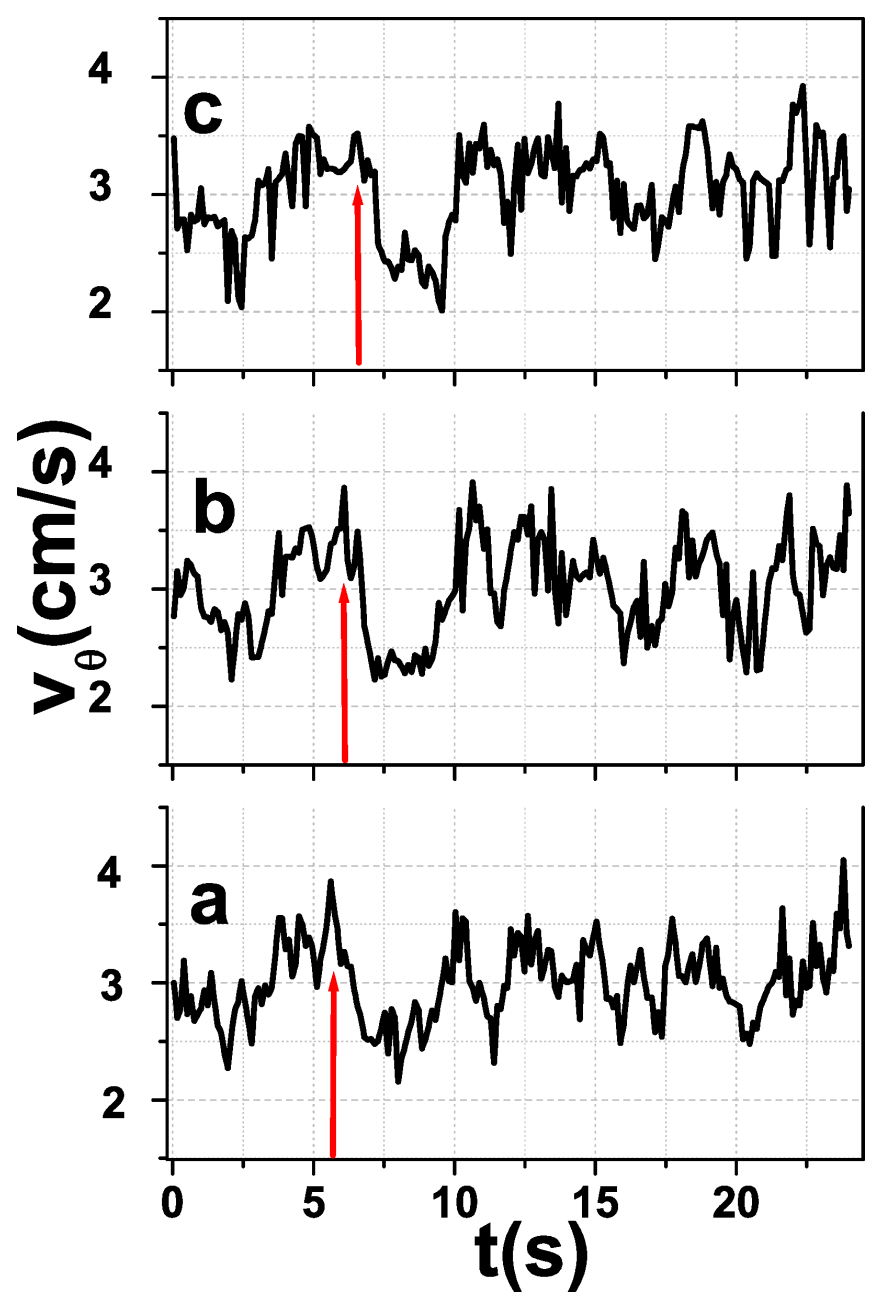

Figure 39. Simultaneous tangential velocity time series (partially shown) at $W i=57.6$ for three values of the displacement along the mean flow line: (a) $s=0 \mathrm{~cm}$, (b) $s=1.5 \mathrm{~cm}$ (c) $s=3.2 \mathrm{~cm}$. 


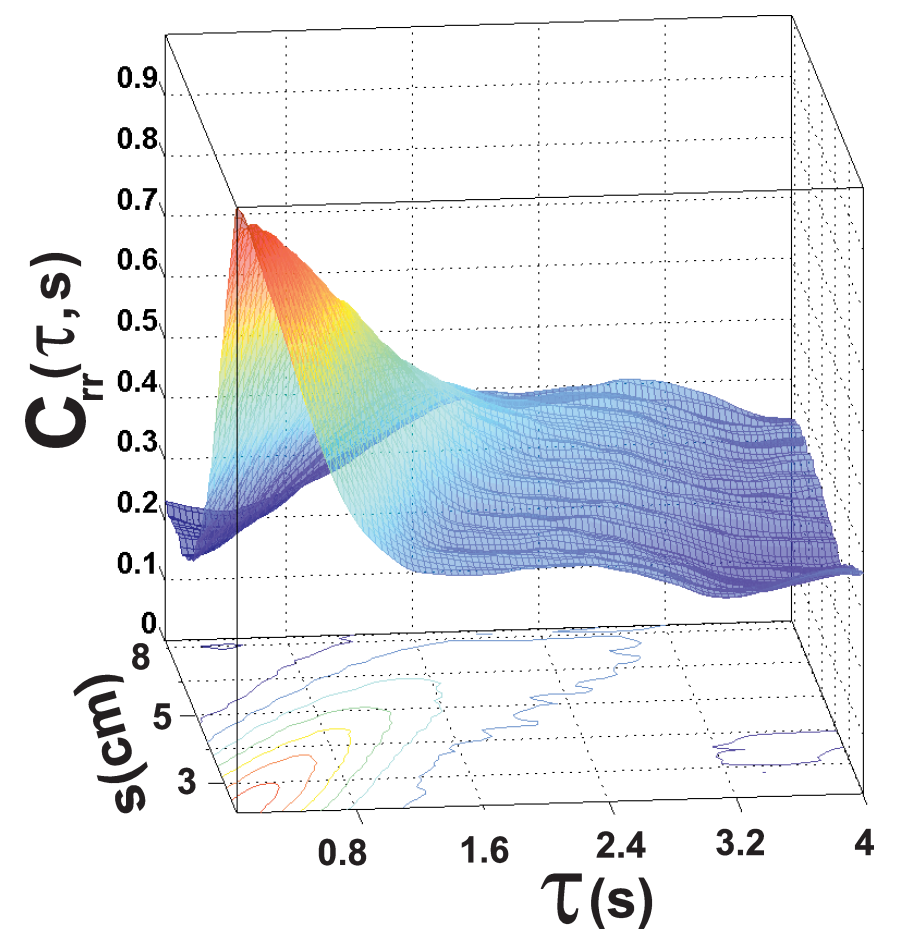

Figure 40. Space -time correlation surface.

For sufficiently small time delays and displacements, the correlation function surface can be well fitted by a paraboloidal surface. Unfortunately, the limited time resolution of the PIV measurements did not allow a full comparison of the coefficients of the expression above with those resulted from the fit. However, the parabolic dependence of $C_{i j}(\tau, \vec{r}, \vec{s})$ on the delay time $\tau$ at a given spatial displacement $s$ or at a given delay time $\tau$ has been verified experimentally. In Fig. 41 we display several cross-correlation functions collapsed onto a single curve being rescaled by the their maxima, $C_{r r}^{\max }$.

Secondly, as resulted from the fit, the second order spatial derivative is about one order of magnitude smaller than the first order one, which is a direct confirmation of the flow smoothness. One can notice that for small enough delay times and space displacements the cross-correlations are well fitted by a parabola as suggested by Eq. 19. Secondly, the weakly parabolic 


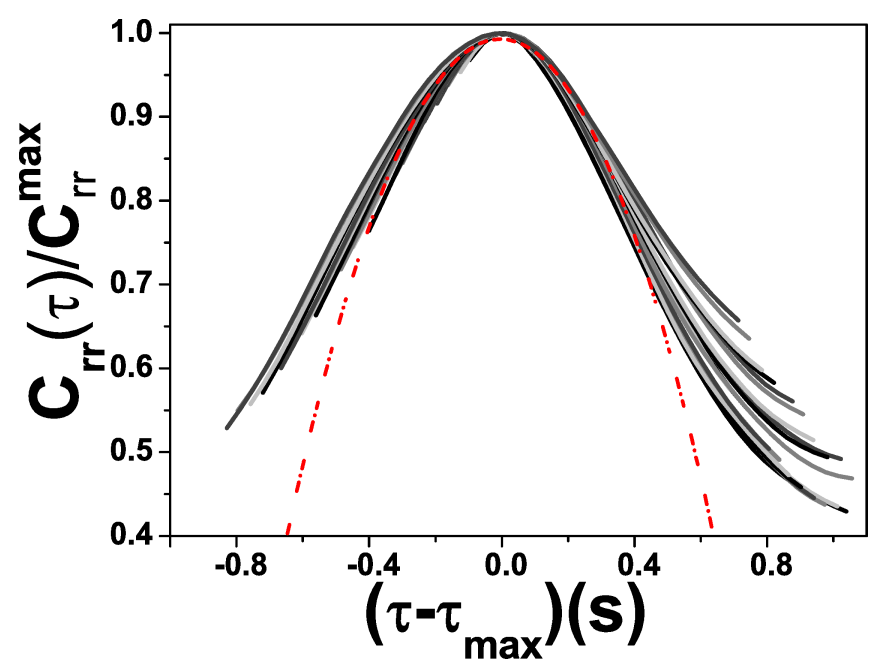

Figure 41. Scaled cross correlation functions for displacements s along the mean flow line ranging from $7 \mathrm{~mm}$ to $4 \mathrm{~cm}$. The dotted line is a parabolic fit.

dependence of $C_{i j}(0, \vec{r}, \vec{s})$ on the displacement $s$ has also been verified experimentally (Fig. 42).

Fig. 43 shows $C_{r r}(\tau, s, \vec{r})$ for few separations $s$ along the mean flow line and corresponding to $\vec{r}=\left(\frac{2 \cdot R_{c}}{3}, 0\right)$.

As one can see in Fig. 43, for a given value of the displacement in space, $\mathrm{s}$, the temporal part of the cross correlation displays a well defined maxima at $\tau_{\max }(s, \vec{r})$, defined by $\left.\frac{\partial C_{r r}\left(\tau_{\max }, s, \vec{r}\right)}{\partial \tau}\right|_{s=\text { const. }}=0$. If $\mathrm{TH}$ is valid and the information on velocity fluctuations is not altered during the passage between two points separated by $s$ in the flow, then the peaks of the temporal part of the cross correlations are sharp and $C_{r r}^{\max }\left(\tau_{\max }\right)$ close to unity. Moreover, the values of the delay time $\tau$ that maximize the correlations should scale linearly with the space displacements, that is $\tau_{\max }=\frac{s}{V}$. Here, as discussed above, in order to correctly account for the flow non-homogeneity, one should consider the spatial dependence of the velocity field, $V=V(\vec{r})$.

Validity of the Taylor hypothesis One way of quantifying the validity of the $\mathrm{TH}$ is to measure the deviation of the slope $p_{1}$ of the dependence 


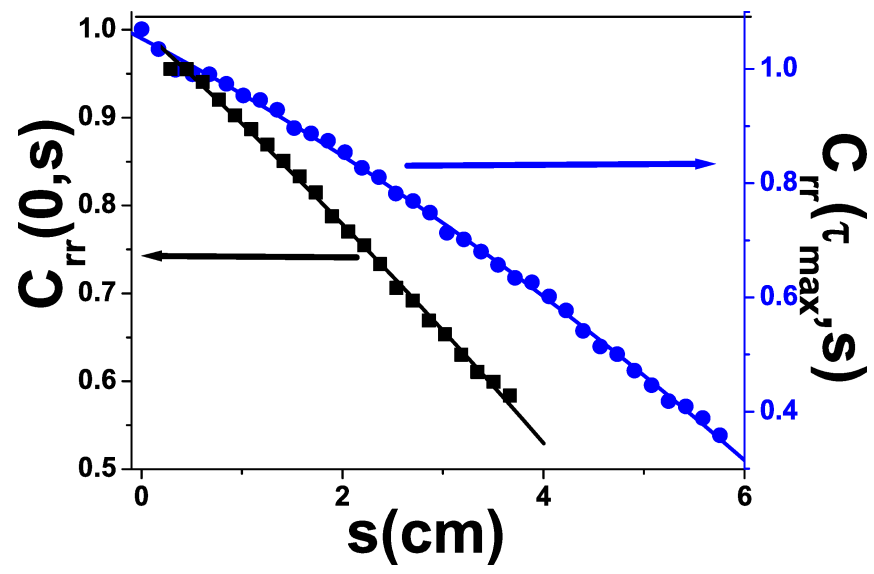

Figure 42. Space dependence of cross correlation functions at fixed time delays: squares- $\tau=0$, circles- $\tau=\tau_{\max }$. The full lines are parabolic fits.

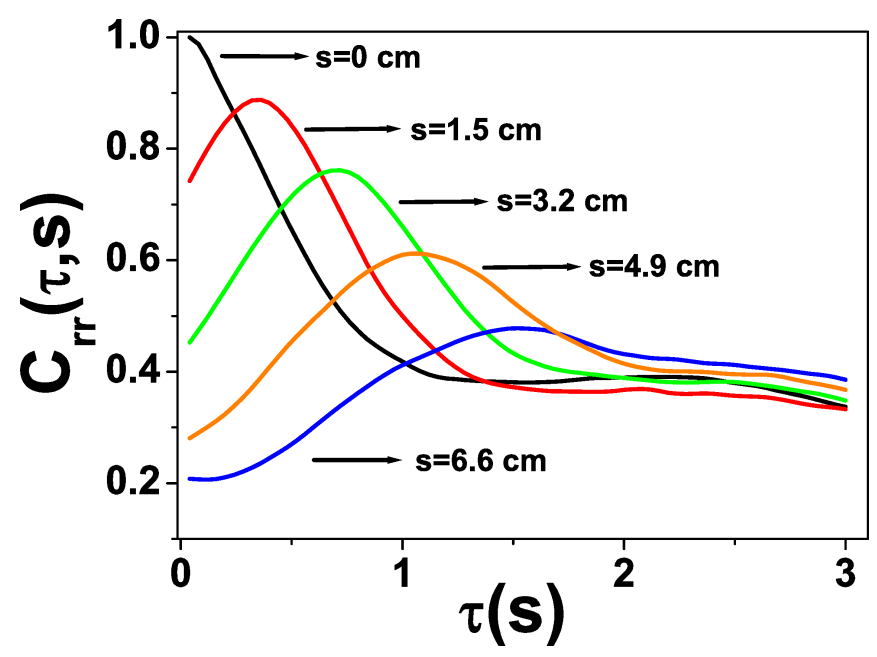

Figure 43. The cross correlation of velocity fluctuations, $C_{r r}(\tau, s)$ vs. the delay time $\tau$ for different values of the displacement $\mathrm{s}$ along the mean flow line. 


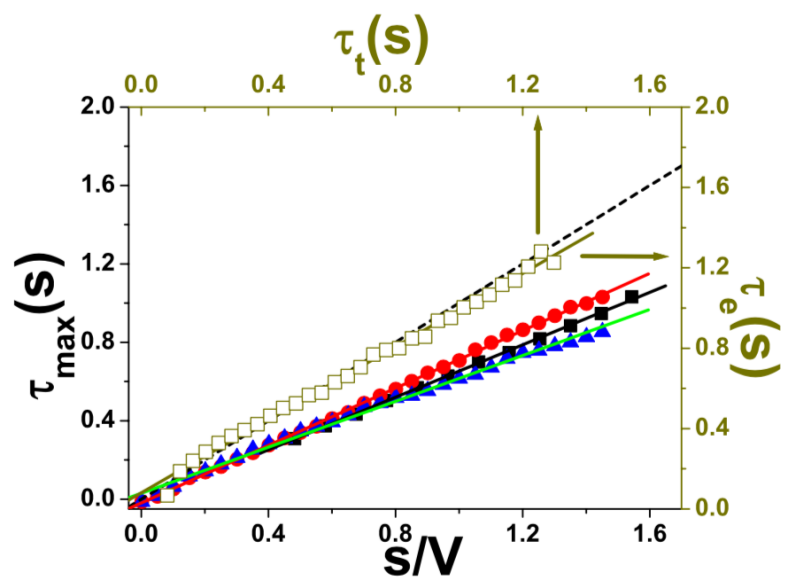

Figure 44. Bottom-left axis: the delay time $\tau_{\max }$ that maximises the cross correlation functions vs. the length of the displacement along three different paths: squares-circular arcs, circles-mean flow line, triangles- Lagrangian trajectory. The horizontal axis is normalised by the mean velocity at $\mathrm{s}=0$. The full lines are linear fits with slopes $0.6,0.7$ and 0.73 respectively. The dotted line has slope 1 . Top-right axis: the delay time $\tau_{e}$ (see text) vs. the transit time $\tau_{t}$. The full line is a linear fit with slope 0.96. The initial position was $\vec{r}=\left(\frac{2 R_{c}}{3}, 0\right)$.

$\tau_{\max }=\tau_{\max }\left(\frac{s}{V}\right)$ from unity Belmonte et al. [2000].

A typical dependence of the maximum delay time $\tau_{\max }$ on the spatial displacement $\mathrm{s}$ is shown in Fig. 44. For this case we have obtained from a linear fit $p_{1} \simeq 0.73 \pm 0.006$.

As one can see from Fig. 45, in the central region of the flow the deviations from the frozen flow assumption are large.

The TH is best verified towards the boundaries of the system. However, $p_{1}$ never exceeds 0.83 .

Whereas the breakdown of the $\mathrm{TH}$ in the central flow region can be easily understood in the context of large (relatively to the mean flow) velocity fluctuations (Figs. 29,30) and practically zero mean flow, the applicability of the frozen flow assumption in the off central flow region deserves a separate discussion. Although chaotic in time, the flow is structurally different from high $R e$ turbulent flows. As we have already pointed out, the main difference 


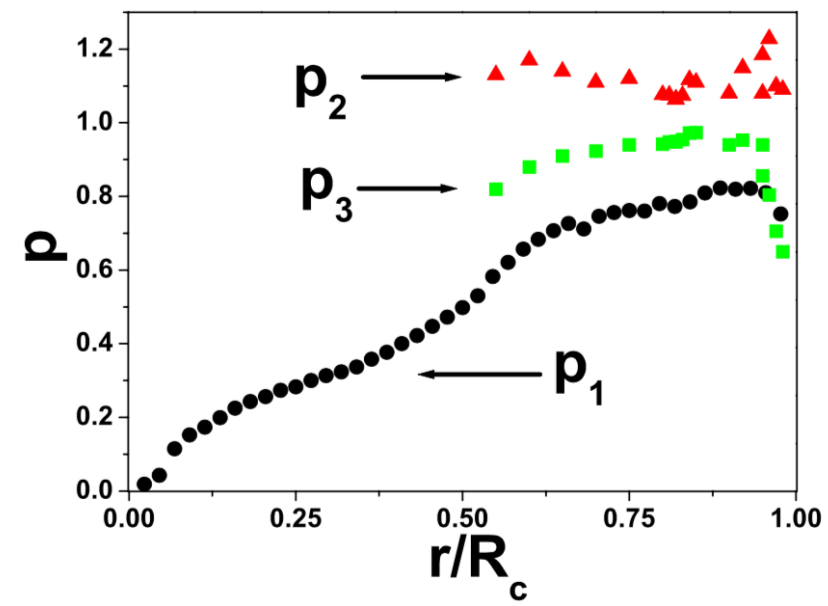

Figure 45. Validity of the TH (see text for discussion) vs. the radial coordinate.

is the absence of any analogue of the Kolmogorov length scale, absence of eddies of different sizes in the scaling range swept by larger eddies, and the flow is smooth at all spatial scales. The main consequence of the flow smoothness is the high space coherence of the velocity fluctuations (the cross-correlations do not decay to 0 even on the size of the container, Fig. $42,43)$.

This fact explains why three physically different choices of the path of particle displacement (circular arcs, mean flow line, Lagrangian trajectories) (Fig. 38), lead to similar values of the maximal delay times $\tau_{\max }$ (Fig. 44 ) .

Secondly, it is worth noting that the validity coefficient $p_{1}$ is always smaller than unity (Figs. 44,45) for each of the three displacement choices we have considered and the deviation from unity (which accounts at best for $20 \%$ ) is clearly outside the error bar of the linear fit. This suggests that the breakdown of the $\mathrm{TH}$ in the off central flow region is not necessarily due to the level of fluctuations (which is the case in the central region) but rather to an underestimation of the advection velocity. Similar results have been previously reported in experimental studies of coherent structures in turbulent jets and turbulent boundary layers Fisher and Davies [1964], Zaman and Hussain [1981], Koeltzsch [1998], Krogstad et al. [1998]. The central 
conclusion of these studies was that the velocity transporting the fluctuation information at scales comparable to the size of the coherent structure is substantially different from the local mean flow velocity. This seems to be indeed the case of the data presented in Fig. 44: if one rescales the displacements $s$ with a velocity about $20 \%$ larger than the mean flow velocity $V$, the data would overlap with the unitary slope line. Consistently with a different choice of the advection velocity Fisher and Davies [1964], Koeltzsch [1998], one can focus on the dependence of $\tau_{e}(s, \vec{r})$ defined implicitly by $\left.\frac{\partial C_{r r}\left(\tau_{e}, s, \vec{r}\right)}{\partial s}\right|_{\tau=\text { const. }}=0$ on the transit time $\tau_{t}$ between two points separated by $s$. If the TH applies, this dependence is linear and has unitary slope.

Thus, a second way to quantify the validity of the frozen flow assumption is to measure the deviation of the slope $p_{2}$ of the dependence $\tau_{e}=\tau_{e}\left(\tau_{t}\right)$ from unity.

Following a suggestion made in Koeltzsch [1998] one can define an additional validity coefficient $p_{3}=\sqrt{p_{1} \cdot p_{2}}$. As shown in Fig. 45 these corrections seem to work rather well for regions away from the vortex core. As an alternative method, the TH can be verified by comparing directly measured spatial structure functions and spatial power spectra of velocity fluctuations with the ones derived using the frozen flow assumption. We now focus on the second order structure function

$$
S_{2}(s)=\left\langle\left|v_{\theta}(\vec{r}, t)-v_{\theta}(\vec{r}+\overrightarrow{\Delta r}, t)\right|^{2}\right\rangle
$$

and compare its values obtained from direct spatial measurements with those obtained using the TH, $s=V \cdot t$.

As shown in Fig. 46, corresponding to the characteristic scale $\mathrm{L}$ the structure functions saturate at values roughly equal to $2 \cdot v_{r m s}^{2}$. Near the core of the main vortex the two structure functions are significantly different [Fig. 46.(a)], indicating once more a clear breakdown of the $\mathrm{TH}$ whereas closer to the boundary [Fig. 46.(b)] the difference diminished to about $20 \%$ of their saturation value that is rather consistent with the result shown in Fig. 45 . 
This part from my HDR needs to be updated

The elastic turbulent flow fields are spatially smooth (meaning that the second-order spatial derivative of the velocity field is roughly an order of magnitude smaller than the first one) and their dominant space scale is set by the size of the fluid container $W$, Burghelea et al. [2007], Burghelea, $\mathrm{T}$ et al. [2005]. Thus, the elastic turbulent flow field may be considered locally linear (i.e. locally uniform rate of strain). Consequently, the spectra of the velocity fluctuations decay algebraically $P \propto f^{-\delta}$ with $\delta \approx 3.5$, Li et al. [2017], Groisman and Steinberg [2000], Burghelea et al. [2007], Burghelea [2005]. The elastic turbulent flows are characterised by divergent Lagrangian trajectories and, consequently, positive finite time Lyapunov exponents (FTLE) could be measured by both numerical integration of the measured velocity fields and by direct tracking of tracer particles in the flow, Burghelea et al. [2004b]. The elastic turbulent flows are highly correlated over space and the characteristic correlation distance is comparable to the size of the fluid container, Burghelea, T et al. [2005]. Systematic experiments performed in a macroscopic von Karman swirling flow between disks by means of both Laser Doppler Velocimetry (LDV) and Digital Particle Image Velocimetry (DPIV) Burghelea et al. [2007] revealed a new characteristic space scale of the elastic turbulence: the width of the stress boundary layer. The Eulerian temporal auto-correlation functions decay rather fast with characteristic correlation times comparable to the longest polymer relaxation time, Burghelea et al. [2007]. From a theoretical standpoint the elastic turbulence remains, in our opinion, an insufficiently explored area and, according to Steinberg [2009], understanding the elastic turbulence might be a fundamental step towards understanding the long standing problem of drag reduction.

From a practical perspective, it has been demonstrated experimentally that elastic turbulent flows are able to mix efficiently very viscous fluids in both macroscopic open channels Groisman and Steinberg [2001] and microchannels Burghelea et al. [2004c,a]. Moreover, the elastic turbulent flows are an ideal realisation of the Batchelor regime of mixing. More recently but equally important from a practical perspective, it has been shown that elastic turbulent flows transfer heat up to four times more efficiently than purely conductive laminar flows, Traore et al. [2015].

\section{Hydrodynamic theory of Elastic Turbulence}

The theory of elastic turbulence of a dilute solution of polymers with linear elasticity and the feedback reaction on the flow was developed by Lebedev et al. Balkovsky et al. [2001], Fouxon and Lebedev [2003]. The distinctive 

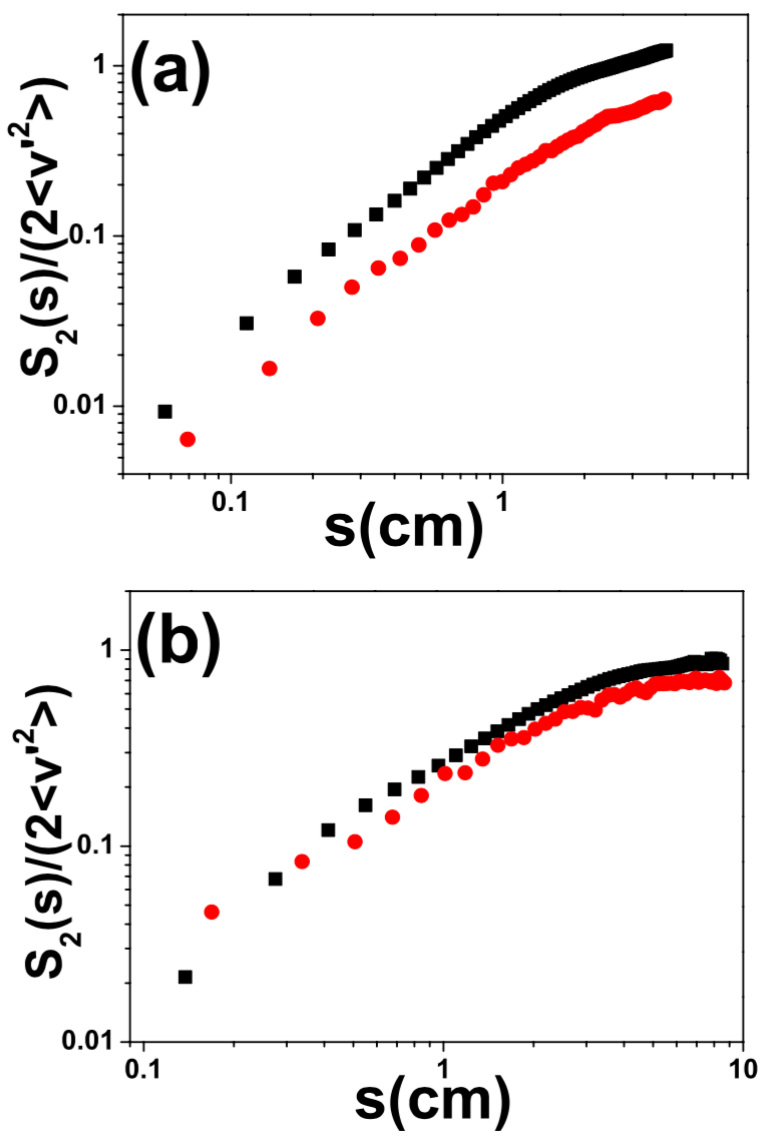

Figure 46. Second order velocity structure functions: squares - resulted from direct measurements, circles derived by using the Taylor hypothesis. Here we have considered displacements along the mean flow line and the initial position was: (a) $\vec{r}=\left(R_{c} / 3,0\right)$ (b) $\vec{r}=\left(2 \cdot R_{c} / 3,0\right)$ 
property of solutions of high molar mass linear polymers is the dependence of the stresses on the flow history, Bird et al. [1977]. Thus, once the flow forcing is removed, the stress decays with a macroscopic relaxation time $\lambda$ (quite often as large as tens of seconds) rather than instantly vanishing. By noting that for a dilute solution the total stress tensor can be decomposed into a solvent and a polymer contribution $\boldsymbol{\sigma}=\boldsymbol{\sigma}_{s}+\boldsymbol{\sigma}_{p}$, the equation of motion takes the form:

$$
\frac{\partial \vec{V}}{\partial t}+(\vec{V} \nabla) \vec{V}=-\frac{\nabla p}{\rho}+\frac{\eta_{s}}{\rho} \Delta \vec{V}+\frac{\nabla \boldsymbol{\sigma}_{p}}{\rho}
$$

The mathematically simplest model accounting for the evolution of the stress tensor in a flow is a single mode Maxwell type constitutive relation:

$$
\boldsymbol{\sigma}_{p}+\lambda \frac{D \boldsymbol{\sigma}_{p}}{D t}=\eta_{p}\left[\nabla \vec{V}+(\nabla \vec{V})^{\dagger}\right]
$$

Here $D \boldsymbol{\sigma}_{p} / D t$ stands for the material time derivative and $\eta_{p}=\eta-\eta_{s}$ is the polymer contribution to the solution viscosity. A commonly used definition of the material time derivative is the upper convective time derivative:

$$
\frac{D \boldsymbol{\sigma}_{p}}{D t}=\frac{d \boldsymbol{\sigma}_{p}}{d t}+(\vec{V} \nabla) \cdot \sigma_{p}-(\vec{V} \nabla)^{\dagger} \cdot \boldsymbol{\sigma}_{p}-\boldsymbol{\sigma}_{p} \cdot(\vec{V} \nabla)
$$

The nonlinear terms in the right hand side of Eq. 23 account for the translation, rotation and stretching of fluid elements and are all of the order of $\lambda \frac{V}{L} \sigma_{p}$.

Equations 22 and 23 are referred to as the Oldroyd-B rheological model for polymer solutions, Bird et al. [1977].

A key ingredient of the theory of the elastic turbulence is to relate the dynamics of the elastic stress tensor $\boldsymbol{\sigma}_{p}$ to the dynamics of a vector field with a linear damping, Fouxon and Lebedev [2003], OGILVIE and PROCTOR [2003], Chertkov [1998], Balkovsky et al. [2001]. This can be done by noting that if one neglects the thermal fluctuations the elastic stress tensor is uniaxial, $\sigma_{p}^{i, j}=B_{i} B_{j}$ which allows one to derive an equation similar to the equation for the magnetic field in magnetohydrodynamics (MHD):

$$
\frac{\partial \vec{B}}{\partial t}+(\vec{V} \nabla) \vec{B}=(\vec{B} \nabla) \vec{V}-\frac{\vec{B}}{\lambda}, \nabla \cdot \vec{B}=0
$$

The stretching of the magnetic field lines is similar to the stretching of polymer molecules in the flow and the sole difference with respect to the MHD case comes from the linear relaxation term $\frac{B}{\lambda}$ which replaces the 
diffusion term. In the absence of inertial contributions, $R e<<1$, the momentum conservation equation can be written:

$$
\nabla P=\rho(\vec{B} \nabla) \vec{B}+\eta_{s} \Delta \vec{V}, \nabla \cdot \vec{V}=0
$$

In the absence of significant inertial contributions $(R e \ll 1)$ which is the case for the elastic turbulence the kinetic energy of the polymer solution can be neglected in comparison with the elastic one. The dissipation of the elastic energy is, however, due to both viscous dissipation due to the solvent viscosity and polymer relaxation:

$$
\frac{d}{d t} \int d \vec{r} \frac{B^{2}}{2}=-\frac{1}{\lambda} \int d \vec{r} \frac{B^{2}}{2}-\frac{\eta_{s}}{\rho} \int d \vec{r}\left(\nabla_{j} V_{i}\right)^{2}
$$

With the appropriate boundary conditions Eqs. 24, 25 exhibit an elastic instability at $W i=W i_{c}$ where $W i=\lambda \dot{\gamma}$ and the instability results in a chaotic and statistically stationary dynamics. Here $\dot{\gamma}$ is the second invariant of the rate of strain tensor.

The theory of elastic turbulence in an unbounded flow of a polymer solution is upon two central assumptions:

1. The local feedback of the stretched polymer molecules on the flow field leads to a statistically stationary state characterised by a saturation of both the polymer contribution to the stress tensor $\tau_{p}$ and the rms of the fluctuations of the velocity gradients $\left(\frac{\partial V_{i}}{\partial x_{j}}\right)^{r m s}$. Consequently, corresponding to a fully developed elastic turbulent regime the local Weissenberg number defined as $W i_{l o c}=\lambda\left(\frac{\partial V_{i}}{\partial x_{j}}\right)^{r m s}$ saturates and, in the bulk of the flow, its saturation value is $W i_{l o c} \approx 1$.

2. Both dissipative terms due to viscosity and polymer relaxation that appear in the equation for the dissipation of elastic energy are of the same order of magnitude $\frac{\sigma_{p}}{\lambda} \approx \frac{\eta}{\rho}(\nabla \vec{V})^{2}$ or, equivalently, $\frac{\sigma_{p} \lambda}{\eta} \approx$ $W i_{\text {loc }}^{2}$.

According to Balkovsky et al. [2001], the first assumption may be phenomenologically understood in the context of a strong back reaction of the extended polymer molecules to the flow as follows. If the instantaneous velocity gradients exceed the reciprocal relaxation time $1 / \lambda$ the polymer coils are stretched which results in an increase of the elastic stresses that damps the velocity gradients. Conversely, if the velocity gradients are much smaller than $1 / \lambda$, the polymer molecules retract and produce no feedback to the flow. Thus, the velocity gradients tend to increase to the characteristic value corresponding to the pure solvent which is significantly larger than 
$1 / \lambda$ above the onset of the transition. The second assumption is consistent with a saturation of the elastic stresses far above the onset of the primary elastic instability.

Next, if one denotes by $\vec{V}^{\prime}$ and $\vec{B}^{\prime}$ the small scale fluctuating parts of the fields $\vec{V}$ and $\vec{B}$ it can be shown that the Fourier components satisfy the linear relationship $\vec{V}^{\prime}=\frac{i \rho(\vec{k} \cdot \vec{B})}{\eta k^{2}} \vec{B}^{\prime}$ which allows one to derive for the spherically normalised spectra of fluctuations of $\vec{V}^{\prime}$ and $\vec{B}$ the following relationships Fouxon and Lebedev [2003]:

$$
\begin{array}{r}
E(k) \propto \eta^{2} L(k L)^{-\delta} \\
F(k) \propto B^{2} L(k L)^{2-\delta}
\end{array}
$$

With the assumptions $\nabla V^{\prime} \ll \nabla V$ and $B^{\prime} \ll B$ it can be shown that $\delta>3$, Fouxon and Lebedev [2003]. The algebraic decay of the velocity spectrum has been measured by means of LDV measurements (and using the Taylor's frozen flow hypothesis was switch from the frequency domain to the wave number domain) in a macroscopic von Karman flow and, subsequently, in a macroscopic serpentine channel by Groisman and Steinberg in Groisman and Steinberg [2000, 2001]. Direct measurements of the spectra in the space domain by means of DPIV revealed a decay exponent $\delta \approx 3.5$, Burghelea et al. [2007], Burghelea [2005]. Contrary to the case of the inertial turbulence, the algebraic scalings of the kinetic and elastic energy are not related to any cascade of energy (or other conserved physical quantity). Because the velocity spectrum decays faster than $k^{-3}$, the dominant space scale is set by the size of the fluid container $L$ and the velocity fluctuations are strongly correlated over distances comparable to $L$. This has been probed experimentally by measurements of the space-time correlation surface in a macroscopic von Karman swirling flow configuration, Burghelea, $\mathrm{T}$ et al. [2005].

A $k^{-1}$ like decay of the spectrum of the fluctuations is characteristic for the decay of a passive scalar in a smooth random flow field in the so called Batchelor regime of mixing, Batchelor [1959b]. Such algebraic decay has been observed for the mixing of a fluorescent tracer by an elastic turbulent flow in both microscopic Burghelea [2005], Burghelea et al. [2004a,c] and macroscopic channel flows Jun and Steinberg [2010]. More recently and somehow unexpectedly a similar spectral decay has been observed for the fluctuations of the temperature in an elastic turbulent von Karman swirling flow cooled from below, Traore et al. [2015]. This indicates that, in spite of the strong temperature dependence of the rheological properties (notably the relaxation time and the shear viscosity) the temperature field behaves 
like a passive scalar. Thus, if the decay exponent of velocity spectrum is $\delta \approx 3$, Eq. 28 suggests that the stress is passively advected by the large scale chaotic smooth flow in a regime of elastic turbulence.

Although many fundamental features of elastic turbulent flows (such as flow smoothness, the algebraic decay of spectra of fluctuations, the decay of the flow correlation) have been investigated experimentally in various macroscopic flows (Couette flow between rotating cylinders, von Karman swirling flow, curvilinear channel flow), the main assumption of the theory concerning with the saturation of velocity gradients still awaits a systematic experimental test.

To this aspect we dedicate the next section.

\section{Characterisation of Elastic Turbulence in microscopic curvilinear flows}

A full space-time characterisation of curvilinear microscopic channel flows of a dilute solution of high molar mass linear polymer in a wide range of $W i$ numbers is, to our best knowledge, still unavailable in the literature. This sets the general scope of this project.

The motivation of focusing on a curvilinear microscopic flow is three - fold. From a fundamental standpoint, we are interested in understanding the role of spatial confinement on the dynamics of the elastic turbulence (and check if the main features observed in macroscopic flows are preserved). From a practical perspective, such flows have already proved their ability to mix efficiently Burghelea et al. [2004c,a] and further practical applications could be found in efficiently transporting heat at micro-scale and enhancing chemical reactions in a lab on a chip environment. Lastly, as compared to their macroscopic counterpart, the microscopic channel flows allow measurements of flow speeds and velocity gradients with both very high spatial and temporal resolution. Of particular interest here is testing the theoretical prediction concerning with the saturation of the velocity gradients in a fully developed elastic turbulent regime, Balkovsky et al. [2001].

Experimental setup and methods The experiments have been performed with a serpentine micro-channel schematically illustrated in Fig. 47 (a). It consists of $N=200$ smoothly interconnected half circular rings with the inner radius $R_{1}=50 \mu \mathrm{m}$ and the outer radius $R_{2}=250 \mu \mathrm{m}$. The width of the micro-channel is $W=R_{2}-R_{1}=200 \mu \mathrm{m}$ and its depth is $H=200 \mu \mathrm{m}$. The geometric aspect ratio relevant to the onset of the primary elastic instability McKinley et al. [1996], Zilz et al. [2012] is $\alpha=R_{1} / W=0.25$.

The micro-channel chip is mounted on an inverted epifluorescent mi- 


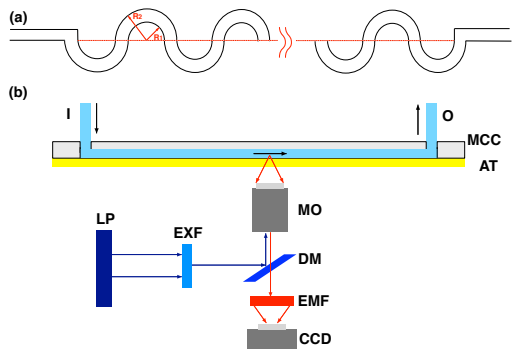

Figure 47. (a) Schematic view of the serpentine micro-channel. (b) Schematic view of the experimental setup (not in scale): MCC - microchannel chip, I - micro-channel inlet, O - micro-channel outlet, AT - adhesive tape, MO - microscope objective, DM - dichroic mirror, LP - led, EXF - excitation filter, EMF - emission filter, CCD - digital camera.

croscope (Nikon Eclipse TS - 100) schematically illustrated in Fig. 47(b). The microscopic flows are generated and controlled by the difference in hydrostatic pressure between the inlet and outlet fluid containers. This is achieved by rigidly mounting the inlet fluid container on a vertical translational stage driven by a precise stepping motor (Isel, Germany). The position of the stage is controlled within $1 \mu \mathrm{m}$ accuracy.

The spatial and temporal features of the flow fields have been investigated by means of a Digital Particle Image Velocimetry technique implemented in house. Particularly important here were both the spatial resolution of the velocity fields $(1.5 \mu \mathrm{m})$ and the total data acquisition time, $T_{\text {total }}=1200 \mathrm{~s}$. This value is roughly 300 times larger than the largest relaxation time of the polymer molecules, $\lambda \approx 4 s$.

\subsection{Onset and development of Elastic Turbulence in micro-channel, flow structure}

A visual assessment of the hydrodynamic stability of the flow is illustrated in Fig. 48 by means of the streak imaging technique. Whereas a laminar flow is observed for low values of the driving pressure $\Delta p=63 \mathrm{~Pa}$ (panel (a)), a strongly irregular fluid motion is observed corresponding to $\Delta p=2.3 k P a($ panel (b)). At this driving pressure a major re-organisation of the flow is apparent in the form of a large scale non-stationary spiral vor- 

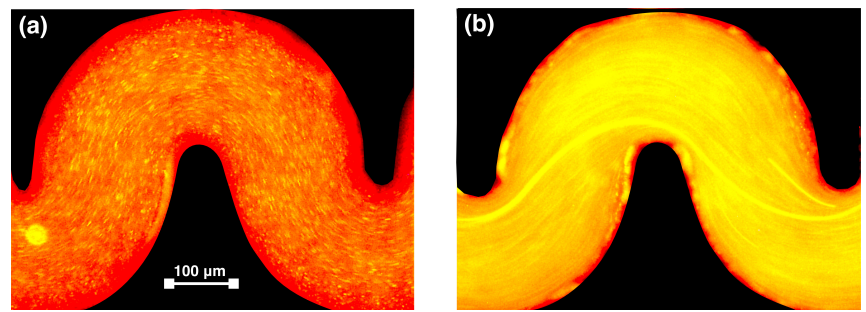

Figure 48. Epifluorescent flow micrographs acquired at (a) $\Delta p=63 \mathrm{~Pa}$ (Wi=0.36 - laminar regime) (b) $\Delta p=2.3 k P a(W i=18$ - elastic turbulent regime). A $X 10$ magnification objective was used.

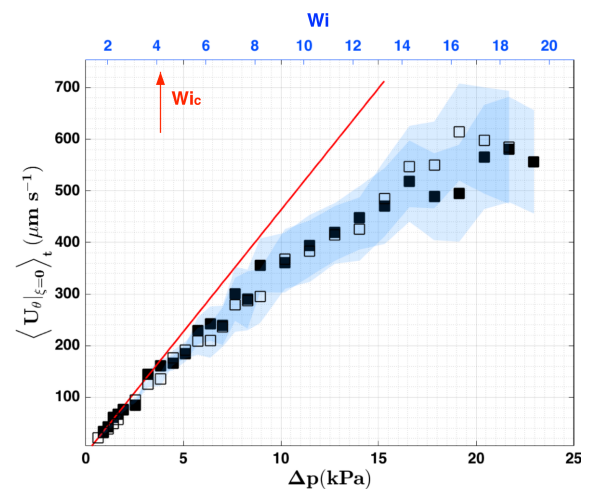

Figure 49. Dependence of the time averaged tangential velocity component on the driving pressure (bottom horizontal axis) and and on the integral Weissenberg number (top horizontal axis). The full/empty symbols refer to the increasing/decreasing branches of the pressure ramp. The shaded regions highlight the level of fluctuations. The full line is a linear fit.

tex in agreement with the observation performed by means of laser scanning confocal microscopy reported in Burghelea [2005]. Measurements of the dependence of the time averaged tangential velocity component measured at the centre line of the channel $(\xi=0)$ on the driving pressure (global Weissenberg numbers) are presented in Fig. 49. The average was performed over 1200 s (a time roughly 320 times longer than the average relaxation time of the polymer $\bar{\lambda}$ ) which guarantees that the flow field information is statistically sufficient.

The dependence of time averaged tangential flow component $\left\langle U_{\theta}\right\rangle_{t}$ on the 


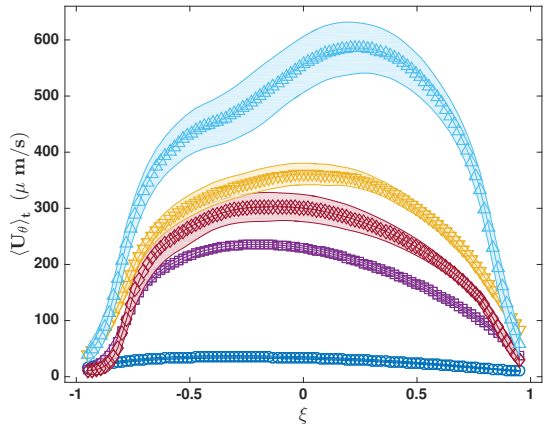

(a)

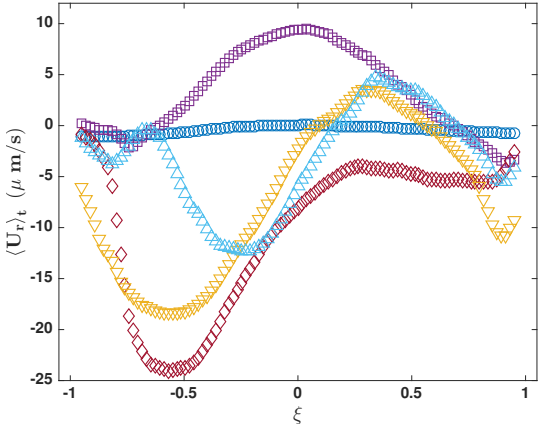

(b)

Figure 50. Time averaged transversal profiles of the tangential velocity component (panel 50(a)) and of the radial velocity component (panel 50(b)). The symbols in panel refer to the driving pressures (global Weissenberg number $): \circ-\Delta p=0.9 k P a(W i=1.1), \square-\Delta p=5.7 k P a(W i=7.4), \diamond$ - $\Delta p=7.6 k P a(W i=9.5), \nabla-\Delta p=10.2 k P a(W i=11.3), \Delta-\Delta p=$ $23 \mathrm{kPa}(\mathrm{Wi}=18.3)$. The shaded regions in panel 50(a) indicate the level of fluctuations of the velocity around its mean.

Weissenberg number remains roughly linear up to a critical value $W i_{c} \approx 4$. Beyond this value, the dependence becomes sub linear indicating a significant re-organisation of the flow associated to an elastic flow instability. It is noteworthy that the transition to elastic turbulence is smooth and, within the instrumental accuracy of the measurements, reversible upon increasing/decreasing pressure drops (the full/empty symbols in Fig. 49). The value of the critical Weissenberg number is comparable to the values reported in Burghelea [2005], Burghelea et al. [2004a,c]. However, a significantly larger discrepancy is found with the onset value given in the recent paper by Jun and Steinberg, Jun and Steinberg [2011], $W i_{c} \approx 200$. This difference can not be explained by the different aspect ratio of the channel $\left(R_{1} / W=1\right)$ using the Pakdel-McKinley scaling, McKinley et al. [1996], Zilz et al. [2012]. As they have used a similar polymer solution, the reasons for this discrepancy remain elusive.

To get further insights into the evolution of the flow structure as the global Weissenberg number is increased we focus on the time averaged transversal profiles of each velocity component, Fig.50.

At low driving pressures corresponding to the laminar flow regime the profile of the tangential velocity component $\left\langle U_{\theta}\right\rangle_{t}$ is non-symmetric (the circles in Fig. 50(a)). Due to the curvilinear geometry of the micro-channel, 


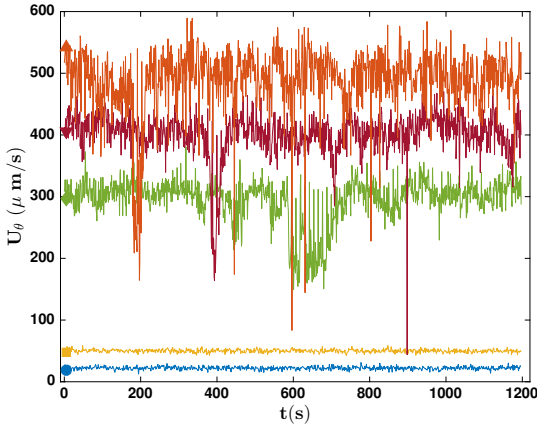

(a)

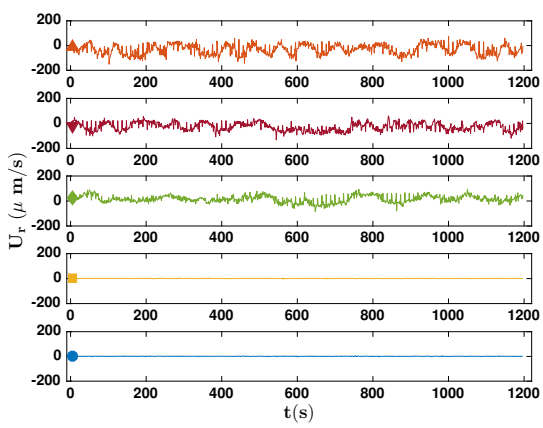

(b)

Figure 51. Time series of the tangential (panel 51(a)) and the radial velocity component (panel 51(b)) measured at the mid-point of the channel $(\xi=0)$ at half depth. The symbols in panel refer to the driving pressure (Weissenberg number): $\bullet-\Delta p=0.9 k P a(W i=1.1), \square-\Delta p=5.7 k P a$ $(W i=7.36), \nabla-\Delta p=7.6 k P a(W i=9.5), \nabla-\Delta p=10.2 k P a(W i=11.3)$, $\Delta-\Delta p=23 k P a(W i=18.3)$.

a non-zero time averaged radial component $\left\langle U_{r}\right\rangle_{t}$ is measured even in a laminar state, Fig. 50(b). An increase of the Weissenberg number past the onset of the primary elastic instability $W i_{c}$ results in a significant reorganisation of the flow: the transversal profiles of the tangential velocity component become more symmetric and a strong inwards radial motion is observed. The flow structure illustrated in Fig. 50 is qualitatively consistent with that observed by the particle tracking velocimetry measurements presented in Ref. Burghelea [2005]. The time series of both the tangential and the radial components of the flow velocity measured at the centre line of the curvilinear micro-channel $(\xi=0)$ are presented in Fig. 51. Within a laminar regime (the time series labelled by a circle and square, respectively), the fluctuations visible in the time series of each velocity component are solely related to the instrumental error of the micro DPIV technique. As the Weissenberg number is increased, the velocity time series exhibit a chaotic like behaviour which reproduces the early measurements reported in Refs. Burghelea et al. [2004c], Burghelea [2005]. As the measurements illustrated in Figs. 51(a), 51(b) are performed over a total time 12 times larger than the acquisition time in Burghelea et al. [2004c], Burghelea [2005] we are now able to observe a new dynamical flow feature in the form of rare "crash" events manifested by a significant slowing down of the flow (see the series labelled by a rhomb and a down triangle in Fig. 51(a)). These rare 


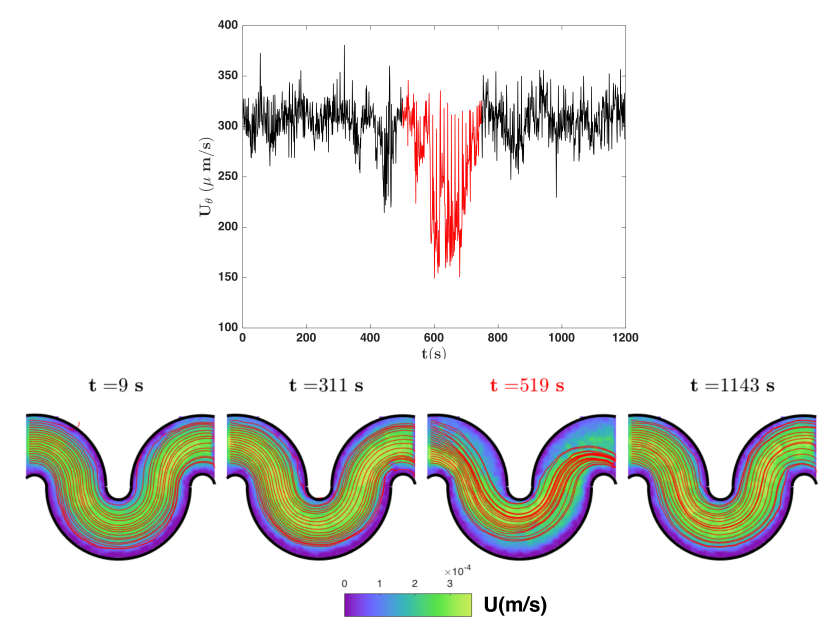

Figure 52. Top: Time series of the tangential velocity component acquired at $W i=9.5$. The bottom plots illustrate maps of the velocity modulus at several time instants indicated on the top. The full lines in each bottom panel are streamlines.

events emerge right above the onset of the primary elastic instability and the frequency of their appearance increases with increasing control parameter.

A closer look into the emergence of rare events near the onset of the elastic turbulence is presented in Fig. 52 by monitoring instantaneous flow fields (the bottom line) before, during and after the emergence of a rare event. One can note that a rare event is associated to a dramatic re-organisation of the flow which persists for times significantly larger than the average polymer relaxation time, $\Delta T \approx 250 \mathrm{~s}$. The emergence of rare events has been previously observed during experiments performed on a macroscopic von Karman swirling flow between two disks of a dilute polymer solution, Burghelea et al. [2007]. In contrast to the observation illustrated in Fig. 52 , in a von Karman flow the rare events are manifested though a local flow acceleration rather than a deceleration, see Figs. 20(a) and 21(a) in Burghelea et al. [2007]. The difference may originate in the different type of forcing of the two flows. Whereas the measurements of the velocity time series in the von Karman swirling flow were performed at a controlled angular speed $\Omega$ of the top disk (which sets the scale for the rate of deformation $\dot{\gamma}$ ) the microscopic flows presented here are driven at constant pressures, i.e. at constant applied stresses. In both cases, however, the frequency of the emergence of rare events increases with increasing global Weissenberg 


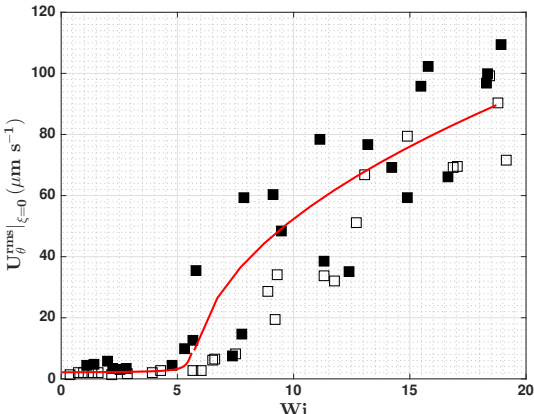

(a)

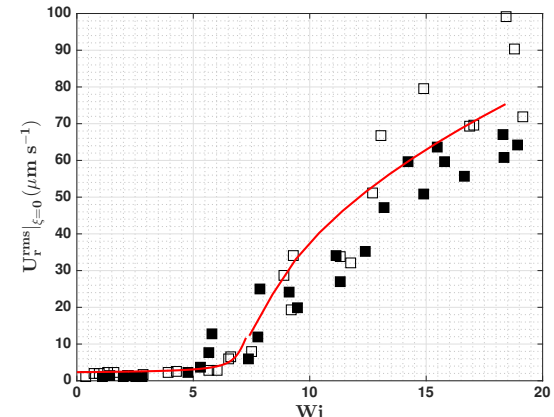

(b)

Figure 53. Dependence of the rms of fluctuations of the tangential velocity component (panel 53(a)) and of the radial velocity component (panel 53(b)) measured at the midpoint of the channel $(\xi=0)$ on the global Weissenberg number $W i$. The full/empty symbols refer to the increasing/decreasing driving pressures. The full line in each panel is a fit by the stationary Ginzburg-Landau equation (see text for description).

numbers indicating a similar physical origin.

\subsection{On the nature of the bifurcation towards Elastic Turbulence}

A next important point relates to the nature of the bifurcation towards elastic turbulent states. To address this point, we focus on the dependence of the rms of fluctuations of each velocity component measured at the centre line of the micro-channel on the Weissenberg number, Fig. 53. A strong increase of the level of fluctuations of both the tangential velocity component Fig. 53(a) and the radial velocity component Fig. 53(b) accounting for up to 35 percent of the mean flow speed is observed.

As already noted for the Weissenberg dependence of the time averaged tangential velocity component presented in Fig. 49, the transition is smooth and, within the level of scatter of the data, reversible upon increasing/decreasing $W i$. Moreover, the data can be fitted by the stationary Landau-Ginzburg equation with a field (the full lines in Figs. 53(a), 53(b)):

$$
\epsilon U_{i}^{r m s}-a\left(U_{i}^{r m s}\right)^{3}+h=0 \quad i=r, \theta
$$

where $\epsilon=W i / W i_{c}-1$ is the reduced control parameter and $a$ and $h$ are fitting parameters. One can conclude that the transition to elastic turbulence in a curvilinear micro-flow occurs via a supercritical bifurcation. This result 
is interesting in itself, somewhat unexpected and it deserves being discussed. In a Taylor-Couette geometry the elastic instability is experimentally found to emerge via a subcritical bifurcation Groisman and Steinberg [1998] in agreement with the theoretical prediction, Sureshkumar et al. [1994]. A systematic and pedagogical description of the subcritical elastic instability in parallel shear flows of viscoelastic fluids is presented in Ref. Morozov and van Saarloos [2007]. The early investigation of the transition to elastic turbulence in a von Karman swirling flow between disks suggested a similar subcritical bifurcation in the form of a hysteresis of the time averaged injected power (calculated as the product between the angular speed of the top disk and the torque that drives it), Burghelea et al. [2007], Burghelea [2005], Burghelea, Teodor et al. [2006]. However, more recent measurements of the injected power into a von Karman swirling flow averaged over significantly longer times reported in Ref. Traore et al. [2015] revealed a smooth and reversible transition consistent with a supercritical bifurcation, see Fig. 4 therein. To conclude this part, the nature of the transition to elastic turbulence remains elusive and future theoretical and numerical studies are needed to clarify this point.

\subsection{Statistics and spatial distribution of the velocity gradients; analysis of boundary layers}

As pointed out in Sec. 5, the key physical quantities that need to be measured in order to verify the predictions of the theory of elastic turbulence are the time averaged fields of the velocity gradients and their level of fluctuation. Particularly, the production of the elastic stresses that destabilise the flow is directly related to the magnitude of the velocity gradients and the level of their fluctuations and one of the key ingredients of the theory of elastic turbulence relates to the saturation of the rms of fluctuations of the velocity gradients due to a strong back reaction of the polymers to the flow. This motivates us to take advantage of the excellent temporal and spatial resolution of the DPIV method described in Sec. ?? to provide a full description of the spatial distribution of the velocity gradients and the long time statistics of their fluctuations.

The spatial distributions of the time averaged second invariant of the rate of strain $\langle\underline{\underline{\underline{\gamma}}}\rangle_{t}$ measured for three distinct Weissenberg numbers are presented in Fig. 54. One can note that the spatial distribution of the second invariant of the rate of strain is strongly in-homogenous and exhibits pronounced local maxima of unequal magnitude (except for the highest $W i$ illustrated in panel (d)) distributed at a constant distance from the inner and outer boundaries of the channel, panel (a) in Fig. 54. As the extension 


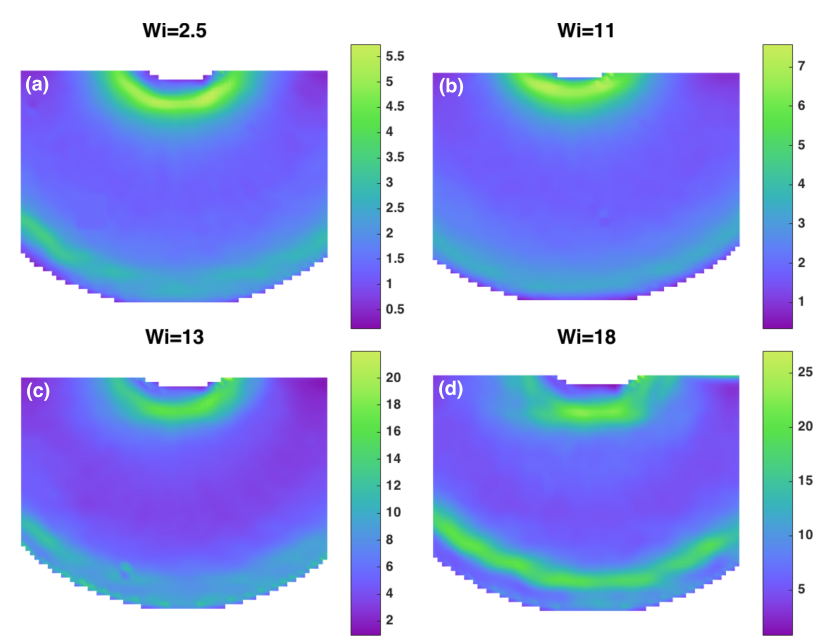

Figure 54. Spatial distribution of the time averaged second invariant of the rate of strain measured at different Weissenberg numbers indicated in the top inserts. The units of the colorbars are $s^{-1}$.

of the linear polymer chains is controlled by the local velocity gradients, we attribute the observation of the local maxima of the velocity gradients to the formation of inner and outer boundary layers of the elastic stresses.

The primary elastic instability is accompanied by a sharp increase of the velocity gradients particularly in the vicinity of the inner boundary of the channel, panel (b) in Fig. 54. The lack of symmetry of the spatial distribution of the velocity gradients with respect to the centre line of the micro-channel indicates a strongly inhomogeneous distribution of elastic stresses along the transversal flow direction. Within a fully developed elastic turbulent regime, the symmetry of the distribution of the elastic stresses with respect to the centre line of the channel is restored. A more systematic investigation of the transversal distribution of the time averaged velocity gradients and of their fluctuations as a function of the global Weissenberg number is presented in Fig. 55.

The emergence of a boundary layer of the elastic stresses when the global Weissenberg number is increased is clearly visible in Fig. 55(a). As already noted in Fig. 54, as one advances within the regime of fully developed elastic turbulence, the two local maxima of the profiles of the time averaged gradients tend to be become equal in magnitude. Corresponding to the edge of the boundary layers, local maxima are also observed in the transversal 


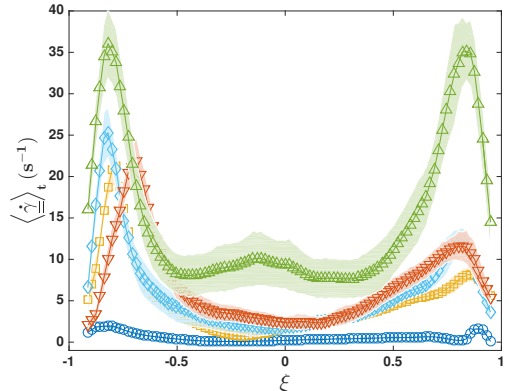

(a)

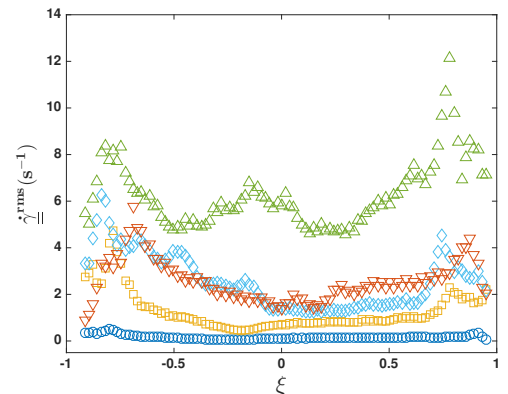

(b)

Figure 55. Transversal profile of the time averaged second invariant of the rate of deformation (panel 55(a)) and of the rms of fluctuations of the second-invariant (panel 55(b)) measured at various driving pressures (global Weissenberg numbers): $\circ-\Delta p=0.9 k P a(W i=1.1), \square-\Delta p=5.7 k P a$ $(W i=7.3), \diamond-\Delta p=7.6 k P a(W i=9.5), \nabla-\Delta p=10.2 k P a(W i=11.3)$, $\triangle-\Delta p=23 k P a(W i=18.3)$. The shaded regions in panel $55($ a) indicate the level of fluctuations around the mean value value.

distributions of the rms of fluctuations of the second invariant of the rate of deformation tensor, Fig. 55(b).

The evolution of the width of the inner and outer boundary layers determined by the location of the peaks of the spatial distribution of the time averaged second invariant of the rate of strain tensor (Fig. 55(a)) with the global Weissenberg number is presented in Fig. 56. The width of the inner boundary layer (the squares) is systematically larger than the width of the outer boundary layer (the circles) up to a Weissenberg number $W i \approx 15$ corresponding to the fully developed elastic turbulent regime when the two boundary layers become practically identical.

We note that, within a regime of elastic turbulence the width of the inner and outer boundary layers is $W_{b l} / W \approx 0.1$ and independent on the global Weissenberg number. This result is consistent with the measurements presented by Jun and Steinberg Jun and Steinberg [2011] and with earlier measurements performed in a macroscopic von Karman swirling flow, Burghelea et al. [2007], Burghelea [2005].

The evolution of the time averaged velocity gradients and of the local Weissenberg number defined as $W i_{l o c}=\bar{\lambda} \dot{\underline{\gamma}}^{r m s}$ measured at the edge of the inner/outer boundary layers (corresponding to the local maxima in Figs. $55(\mathrm{a}), 55(\mathrm{~b}))$ and in the bulk of the flow (averaged around the centre line 


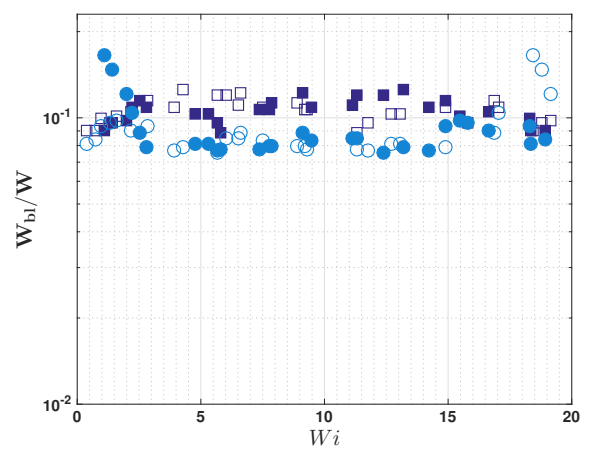

Figure 56. Dependence of the reduced width of the inner boundary layer $(\square, \boldsymbol{\square})$ and of the outer boundary layer $(\bullet, \circ)$ on the global Weissenberg number. The full/empty symbols refer to increasing/decreasing global Weissenberg numbers, respectively.

of the micro-channel) with the global Weissenberg number is presented in Fig. 57 .

The primary elastic instability is accompanied by a sharp increase of both the time averaged velocity gradients (panel 57(a)) and of the local Weissenberg number (panel 57(b)). For $W i \leq 17$ the time averaged velocity gradients measured at the edge of the inner boundary layer are systematically larger than those measured at the edge of the outer boundary layer, Fig. 57(a). The local Weissenberg number measured at the edge of the inner boundary layer is systematically larger than the one measured at the centre line of the flow, Fig. 57(b). Within a fully developed elastic turbulent regime the local Weissenberg number measured at the edge of the boundary layers increases linearly with the global Weissenberg number $W i_{l o c}^{b l}=-25.58+3.54 W i$ (the dashed line in Fig. 57(b)). On the other hand, a linear fit of the local Weissenberg number measured in the bulk of the flow gives $W i_{\text {loc }}^{b u l k}=15.21+0.12 W i$ (the full line in Fig. 57(b)). Thus, one can conclude that in a fully developed elastic turbulence regime $W i_{l o c}$ saturates in the bulk of the flow but keeps increasing at the edge of the boundary layers. The result is in a qualitative agreement with the theoretical prediction for the elastic turbulence, Fouxon and Lebedev [2003], Balkovsky et al. [2001]. Corresponding to the bulk measurements one obtains $W i_{\text {loc }}^{\text {bulk }} \approx 15.5$ which is significantly larger than the unitary value predicted theoretically indicating that no quantitative agreement with the theory is found. A similar conclusion has been reached for the case of an elastic turbulent flow in a macroscopic von Karman swirling flow configu- 


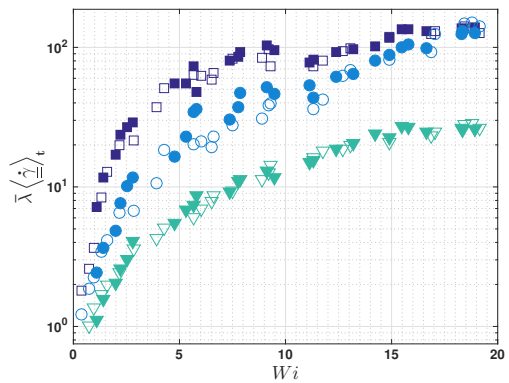

(a)

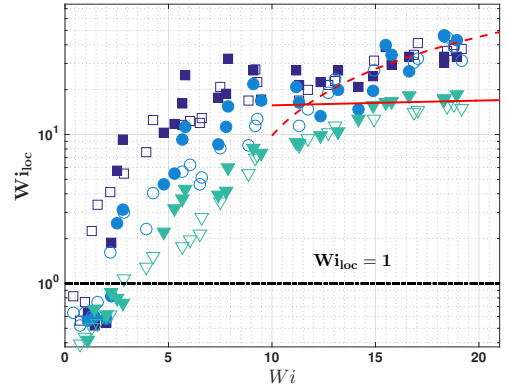

(b)

Figure 57. 57(a) Dependence of the time averaged second invariant of rate of deformation on the global Weissenberg number. 57(b) Dependence of the local Weissenberg number $W i_{l o c}$ on the global Weissenberg number. The full and dashed lines are linear fit functions (see text for description). In both panels the symbols refer to different radial locations $\xi$ of the measurements: $(\square, \boldsymbol{\square})$ - the edge of the inner boundary layer, $(\bullet, \circ)$ - the edge of the outer boundary layer, $(\mathbf{\nabla}, \nabla)$ - average in the bulk of the flow within the range $\xi \in[-0.4,0.4]$. In both panels the full/empty symbols refer to increasing/decreasing global Weissenberg numbers, respectively.

ration, Burghelea et al. [2007], Burghelea, Teodor et al. [2006], Burghelea [2005]. The local Weissenberg number was defined in these references as $W i_{l o c}=\omega^{r m s} \lambda$ (here $\omega$ stands for the vorticity) saturates at the value $W i_{l o c} \approx 2$ (Fig. 19(b) in Burghelea et al. [2007], insert of Fig. 2 in Burghelea, Teodor et al. [2006]) which is twice larger than the value predicted by the theory. We conclude that the main assumption of the theory of elastic turbulence concerning with the saturation of the velocity gradients is verified only qualitatively. Quantitatively, the experiments revealed a systematic discrepancy which is more pronounced in a microscopic flow than a macroscopic one. This aspect may originate in the strong spatial confinement of the microscopic flow and needs to be addressed by further theoretical developements.

The result on the saturation of the local Weissenberg number in the bulk of the flow is at odds with the recent measurements reported in Jun and Steinberg [2011] which indicate a linear increase of $W i_{l o c}$ with $W i$ rather than a saturation.

Finally, we note that the measurements of the time averaged velocity gradients and of the rms of their fluctuations are reproducible upon increasing/decreasing $W i$ proving once more the reversibility of the bifurcation 
towards elastic turbulence (the full/empty symbols in Fig. 57) in a curvilinear micro-channel flow in full agreement with the behaviour observed in Figs. 49,53.

\section{Efficient mixing by Elastic Turbulence}

Microscopic flows have been attracting an increasing interest during the past decade due to fast development of micro-fluidics and soft lithography Whitesides and Stroock [2001]. The use of micro-fluidic devices has few key practical advantages such as the dramatic reduction of the amount of reagents required for fine chemistry and biochemistry Hansen et al. [2002] applications, well controlled manipulation and sophisticated experiments on single cells Li Jeon et al. [2002], Takayama et al. [2001], Mao et al. [2003] and macromolecules Chou et al. [1999]. The microscopic flows are almost universally laminar, steady and characterised by a linear dependence of the flow rate on the driving force. These basic characteristics are determined by low values of the Reynolds number, $R e$, which is a general measure of the inertial nonlinearity of the flow Landau and Lisfshits [1980]. Here, $V$ is the mean flow velocity, $\mathrm{d}$ is the diameter of the channel, and $\rho$ and $\eta$ are the density and the viscosity of the fluid, respectively. When $d$ is reduced, the flow velocity needed to reach a high enough value of Re in order to excite chaotic or turbulent states increases as $d^{-1}$ and the required driving pressure (at fixed aspect ratio) as $d^{-2}$. Therefore, when channels are only few tens of microns wide, achieving high Re requires impractically high driving pressures. A main consequence of the laminar character of the flow is the inefficient mass transfer across the main flow direction which occurs mainly due to molecular diffusion. Mixing of viscous fluids by diffusion is slow comparative to the mixing that occurs in a random flow. Even for a moderate size protein, such as bovine serum albumin (with a diffusion coefficient $D \approx 3 X 10^{-7} \mathrm{~cm}^{2} / \mathrm{s}$ in water), the diffusion time $d^{2} / D$ across a micro channel with a width of $100 \mu \mathrm{m}$ is of the order of $100 \mathrm{~s}$.

Few techniques have been suggested to generate stirring by a threedimensional flow in order to increase the mixing efficiency in micro-channel flows. They include application of time-dependent external force fields Oddy et al. [2001], Tsai and Lin [2002] and increasing Re to moderately high values in curvilinear three-dimensional channels Therriault et al. [2003], Vijayendran et al. [2003]. An ingenious solution to generate chaotic advection in a microscopic flow was suggested in Stroock et al. [2002]. It involves a special "herringbone" patterning of a micro-channel wall which enhances a secondary flow normal to the mean flow direction. The continuous stretching and folding of the fluid elements (as they advance downstream) results in 
exponential separation of initially close fluid particles and efficient mixing. However, the flow was stationary in laboratory frame.

Groisman and Steinberg have demonstrated demonstrates that a small addition of high molecular weight linear polymer to a macroscopic curvilinear flow of a viscous fluid can lead to very efficient mixing in a regime of elastic turbulence Groisman and Steinberg [2001].

If the size of the flow channel is reduced to a microscopic scale, the extension of polymer molecules in the flow may become comparable to the size of the setup. Therefore, the question whether a microscopic flow of a dilute polymer solution can undergo a purely elastic instability and evolve towards random flow states (in a regime of elastic turbulence) can not be answered by a simple analogy with the macroscopic case and needed to be addressed experimentally.

This section is organised as follows. The first section describes the microscopic flow system and the experimental investigation techniques. The transition to random flow and the main features of the random micro-flow are discussed in the second section. In the third section I present a detailed investigation of the decay of a passive tracer in the random micro-flow. The slow down of mixing with respect to the case of unbounded systems (Batchelor regime) is further clarified by an analysis of the mixing boundary layer.

\subsection{The polymer solutions.}

The polymer solution used in this series of experiments was identical to that used in the experiments presented in the previous Chapter, that is 80 ppm PAAm dissolved in a Newtonian solvent(about $65 \%$ saccharose in water). The viscosity of the solution was $\eta=138 \mathrm{mPas}$ at a shear rate of $2 s^{-1}$ and the viscosity of the solvent was $\eta_{s}=114 \mathrm{mPas}$ at $22^{\circ} \mathrm{C}$. The polymer relaxation time $\lambda$ is shown as a function of shear rate in Fig. 1.2.

The overlap concentration $c^{\star}$, defined as the concentration at which the viscosity ratio $\frac{\eta}{\eta_{s}}=2$, was $200 \mathrm{ppm}$, corresponding to a molecular concentration $n=8.76 \times 10^{12} \mathrm{~cm}^{-3}$. The characteristic size of polymer molecules at rest can be estimated from this as $n^{-1 / 3} \simeq 0.5 \mu \mathrm{m}$, and the characteristic distance between them at $80 \mathrm{ppm}$ by weight can be estimated as $0.7 \mu \mathrm{m}$. These estimates are well supported by the data on PAAm taken from the literature Kulicke et al. [1989]. On the other hand, one can use the Mark-Houvink scaling relation for PAAm, $[\eta]=6.31 X 10^{-3} M_{w}^{0.8}$ (in $\mathrm{ml} / \mathrm{g}$ ) Kulicke et al. [1989], where $[\eta]$ is the intirinsic viscosity of the polymer defined as $[\eta]=\lim _{c \rightarrow 0} \frac{\eta-\eta_{s}}{c \cdot \eta_{s}}$. Corresponding to the molecular weight $M_{w}=1.8 \times 10^{7} \mathrm{Da}$ one obtains $[\eta]=4020 \mathrm{ml} / \mathrm{g}$. Defining $c^{\star}=[\eta]^{-1} \operatorname{Bird}$ 


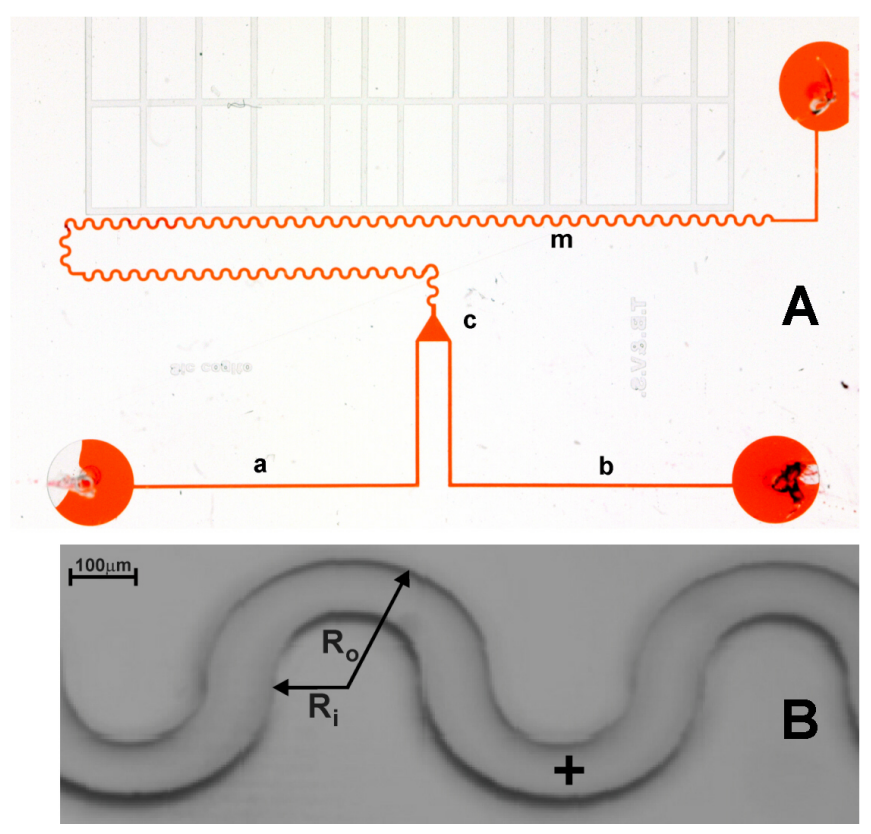

Figure 58. (A) Photograph of the micro-fluidic device. The micro-channel was filled with ink for better contrast. (B)Photograph of a section of the functional curvilinear element. The point where instantaneous flow velocity measurements (averaged over a 20X20 $\mu \mathrm{m}$ square region) were made is marked by a cross.

et al. [1977] we obtain $c^{\star}=250 \mathrm{ppm}$ by weight for an aqueous solution which agrees well with the above estimate.

Additionally, one can compare the estimated size of the PAAm coils with the data obtained from light scattering, Ref. Kulicke et al. [1989]. Plugging $M_{w}=1.8 \times 10^{7} \mathrm{Da}$ into an interpolation relation from Ref. Bird et al. [1977], one obtains $R_{g} \simeq 0.4 \mu \mathrm{m}$ for the radius of gyration of the coils, which is rather consistent with the above estimate obtained from $c^{\star}$.

It is worth noting that $c^{\star}=200 \mathrm{ppm}$ is very close to the value found for $\lambda$-phage DNA Shrewsbury et al. [2001], which has a comparable molecular weight of $3.1 X 10^{7} \mathrm{Da}$. Radius of gyration of $\lambda$-phage DNA was found to be $0.73 \mu \mathrm{m}$ Smith et al. [1996], which is quite consistent with the above estimates for the PAAm coil size. The full contour length of a PAAm molecule having $M_{w}=1.8 \times 10^{7} \mathrm{Da}$ and thus consisting of $2.5 \times 10^{5}$ monomers (each with the molecular weight $71.08 \mathrm{~g} / \mathrm{mol}$ ) can be estimated as about $50 \mu \mathrm{m}$, 
if a monomer length of $0.2 \mathrm{~nm}$ is assumed. It is significantly larger than the contour length of the $\lambda$-phage DNA (which is equal to about $16 \mu \mathrm{m}$ ) and twice smaller than the width of the micro-channel. The simple considerations presented above suggest that a direct comparison with the case of macroscopic curvilinear flows in a regime of elastic turbulence, Groisman and Steinberg [2001], is not justified and the question whether elastic turbulent states can be excited in a microscopic channel had to be addressed experimentally.

\subsection{Passive scalar field measurements}

The mixing experiments have been conducted by evenly injecting two streams of dilute polymer solution one of which contained also a small amount of fluorescent dye (Fig. $61 \mathrm{~A}$ ). The mixing efficiency is studied at different locations downstream (or versus time, if one assumes that Taylor hypothesis is valid for this flow ${ }^{11}$ ) by monitoring the brightness of the passive scalar field. A critical requirement during the experiments was to visualise the mixing patterns in a narrow horizontal plane, in order to avoid space averaging which could lead to wrong conclusions on the mixing efficiency.

This is achieved by imaging the passive scalar fields through a Confocal Scanning System (Fluoview FV500 by Olympus), Fig. 59. It was equipped with a $40 X, N A=0.85$ infinity corrected objective and a 12-bit photomultiplier. The scanning was done at a rate of 56 lines per second and 512 pixels per line corresponding to a step of $0.18 \mu \mathrm{m}$ per pixel.

Prior to discussing the decay regime of mixing in a random microscopic flow, we illustrate the chaotic flow structure as revealed by confocal scanning microscopy, Fig. 60.

As already discussed in detail in Sec. 6 , the flow is dominated by a large scale vortex evolving randomly in time.

\subsection{Decay regime of mixing in a random micro flow}

Mixing of a low diffusivity passive tracer by a turbulent flow particularly in a region of small scales, attracted recently significant attention from both theoryShraiman and Siggia [1994], Chertkov et al. [1995b], Shraiman and Siggia [2000], Falkovich et al. [2001], Son [1999] and experiment Jullien et al. [2000], Groisman and Steinberg [2001].

\footnotetext{
${ }^{11}$ A detailed discussion of the validity of the Taylor Hypothesis in a similar flow was presented in section 1.5.
} 


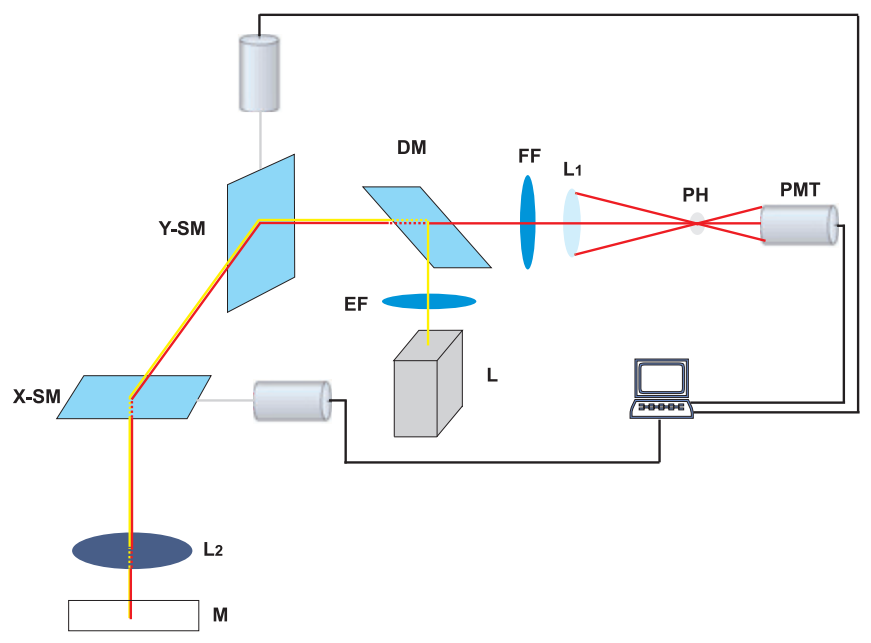

Figure 59. Confocal scanning system: PMT-photo-multiplier, PH-pin hole, L1-focusing lens ,FF-fluorescence filter, DM-dichroic mirror, EFexcitation light selection filter, L-laser, Y-SM-Y direction scanning mirror, X-SM-X direction scanning mirror, L2-objective lens, M-micro-channel.

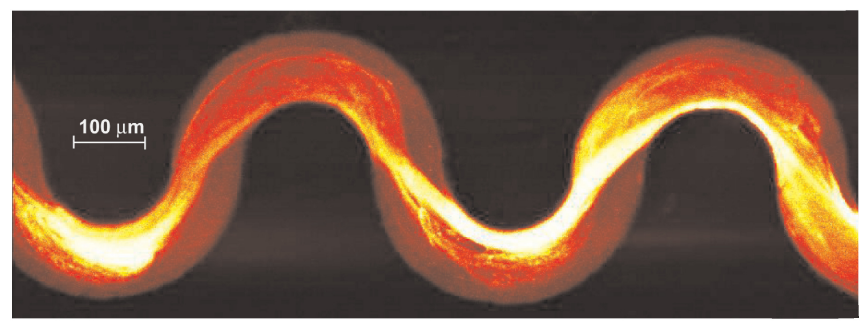

Figure 60. Middle plane horizontal confocal snapshot. The flow is seeded with $0.2 \mu \mathrm{m}$ fluorescent spheres. The driving pressure is $120 \mathrm{~Pa}$ per channel segment

This so-called Batchelor regime of mixing exists in a viscous-convective range of wave numbers, $k_{K}<k<k_{B}$, where the random-in-time velocity field can be treated as smooth. Here $k_{K}=\left(\epsilon / \nu^{3}\right)^{1 / 4}$ is the Kolmogorov dissipation scale, $k_{B}=\left(\epsilon / \nu D^{2}\right)^{1 / 4}$ is the Batchelor wave number, $\nu$ and 
$D$ are the kinematic viscosity and the diffusion constant, respectively, and $\epsilon$ is the rate of energy dissipation Batchelor [1959a]. The inverse injection scale should be used here instead of $k_{K}$, if the former is larger than $k_{K}$. The range of the Batchelor regime is defined by the value of the Schmidt number $S c=\nu / D$, which can be rather large for a viscous fluid with a low diffusivity tracer. The smoothness of the velocity field for developed turbulence below the Kolmogorov scale is explained by an exponential decay of the velocity spectrum there. The Batchelor-Kraichnan approach Batchelor [1959a], Kraichnan [1968] can be extended to an algebraic type velocity spectrum $\propto k^{-\delta}$ with $\delta>3$. Then fluctuation spectrum of the velocity gradients scale as $k^{-\delta+2}$, so that the flow becomes increasingly homogeneous on small scales, and the mixing occurs mainly due to eddies corresponding to the border of the velocity smoothness. Batchelor and Kraichnan Batchelor [1959a], Kraichnan [1968] considered a stationary regime of turbulent mixing with a tracer continuously injected into a turbulent flow on a large scale. Then the mixing is produced by stretching and folding of a tracer blob by the random velocity field creating components of higher and higher wave numbers up to $k_{B}$. A more recent theory of the passive scalar decay in the Batchelor regime of mixing, which is more relevant to a real experiment, makes predictions about the statistics of the passive scalarSon [1999].

Various theoretical predictions for the stationary and decay cases of the Batchelor regime of mixing were verified recently in macro-systems Jullien et al. [2000], Groisman and Steinberg [2001]. However, the theories mentioned Batchelor [1959a], Chertkov et al. [1995b], Son [1999] consider an unbounded case only. As shown by Chertkov and Lebedev, Chertkov and Lebedev [2003], a non-uniform velocity distribution particularly close to a wall, can alter significantly the efficiency of mixing in the decay regime due to turbulent advection. Indeed, due to a reduced velocity near the wall the boundary layer becomes a sink for the passive tracer. This excess of the tracer is intermittently injected from the boundary layer into the bulk. Thus, the tracer decay, e.g. along a channel, is controlled by the rate of tracer injection from the boundary layer that caused a significant slow down of the decay compared with an unbounded case. As a result, the scaling of the mixing length with the Péclet number, $P e=V_{a v} d / D$, changes from logarithmic one to an algebraic, $L_{m i x} \propto P e^{1 / 4}$. Here $P e$ defines the relative rate of advection versus diffusive transport in a flow, $V_{a v}$ is the average longitudinal velocity, and $d$ is the channel width. Besides, the boundary layer width for mixing in the decay regime in scales as $W \propto P e^{-1 / 4}$.

As demonstrated in Sec. 6, above the onset of the elastic instability the micro-flow is spatially smooth and dominated by a large scale randomly fluctuating spiral vortex Fig. 60. The small scale homogeneity of the micro- 
flow suggests that the mixing of a passive tracer injected at large scale in the micro-flow should occur mainly due to the large scale eddies in a Batchelor regime. 

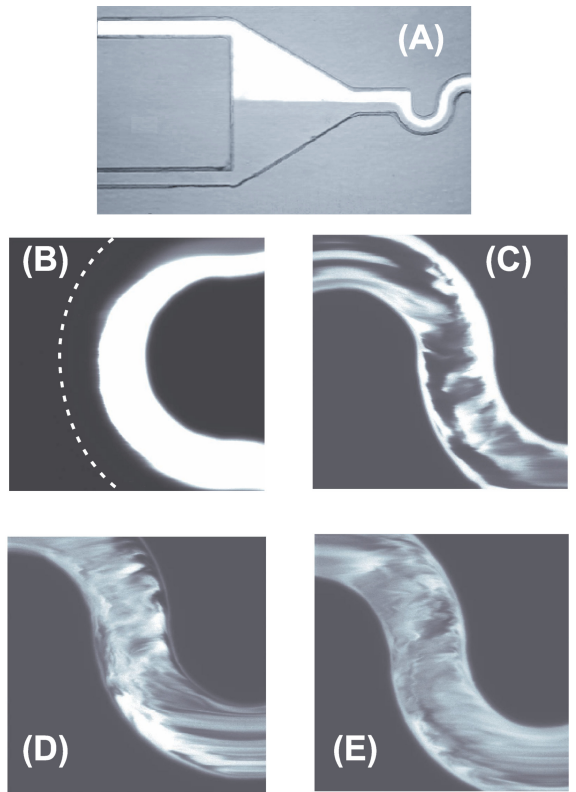

Figure 61. (A)Epifluorescent microphotograph of the entrance area of a micro-channel. Wide triangular region in front of a curvilinear channel allows to adjust equal flow rates for polymer solutions with (from the top) and without FITCD. (B)Confocal micrograph of the flow in the microchannel (at $\mathrm{N}=30$ ) without polymers added. Left wall of the channel is shown by a dotted line. Confocal images of mixing in a random micro flow of a dilute polymer solution at different locations downstream: (C)N=5 (D) $\mathrm{N}=11(\mathrm{E}) \mathrm{N}=17$.

\subsection{Scaling of the mixing length with $\mathrm{Pe}$}

The small scale homogeneity of the micro flow emphasised in the previous subsection suggests that the mixing of a passive scalar injected at large scales in the flow should occur mainly due to the large scale eddies in a so called Batchelor regime of mixing.

Although the Batchelor regime in unbounded systems has been intensively studied theoretically, it has been shown only recently Chertkov and Lebedev [2003] that the mixing efficiency is significantly altered in bounded systems where the boundary layer becomes a sink for the passive tracers. In order to check the theoretical predictions made in Chertkov and Lebe- 
dev [2003], we have varied Pe about 37 times by using fluorescent tracers with different diffusion coefficients and by varying the mean flow velocity. The mixing experiments have been carried out by injecting two streams of polymer solution, one with and one without a fluorescent tracer. The two streams of polymer solution have been injected at equal flow rates by a careful adjustment of the driving pressures, Fig. $61 \mathrm{~A}$.

As a passive tracer we have used dextran molecules labelled with fluorescein isothiocyanate (FITCD). The molecular weights of the passive tracers used in different experiments were $10 k D a, 70 k D a, 500 k D a$ and $2 M D a$. These different choices of tracers together with variations of the mean flow velocity allowed us to modify $P e$ about 37 times in the range $1.6 \times 10^{4} \div 4.14 \times 10^{5}$. As a result, the Batchelor scale, $\eta_{B}=d \cdot P e^{-\frac{1}{2}}$, changed from $1 \times 10^{-4}$ down to $0.15 \times 10^{-4} \mathrm{~cm}$.

First, the setup was tested by running experiments with the plain solvents without PAAm added. The flow appeared laminar and steady in the full range of driving pressures, $\Delta p_{s}$, and the interface between the streams with and without FITCD remained smooth and sharp along the whole channel with only a minor smearing by diffusion Fig. 61.B.

The situation was similar when polymer solutions have been injected in the linear regime corresponding to low values of the driving presures, $\Delta p_{s}$. However, when the driving pressure was raised above the nonlinear transition threshold, $\Delta p_{s}^{\star}=50 \mathrm{~Pa}$ (which corresponds to $W i_{c} \simeq 6.5$ ), the randomly fluctuating flow velocity produced significant stirring, complex and chaotically changing tracer fields. In Fig. 61.C-E are displayed typical scalar fields at different locations downstream. One can easily see that, as one advances downstream, the scalar field has an increasingly random appearance.

In Fig. 62 is shown the distribution of tracer concentration in the vertical cross section of the micro-channel at different locations downstream. Close to the entrance of the channel (62 A-C), the mixing is mainly carried out at large scale by a randomly fluctuating vortex. As one advances downstream, the random stretching and folding of fluid elements results in an increasingly efficient mixing at small scales. The confocal scans in a horizontal plane, Fig. 61B-E, were made at a scanning rate of 56 lines per second, contained 750 individual lines, and took about $13 \mathrm{~s}$ to complete. The vertical scan, Fig. 62B-E was performed with a scanning rate of 24 lines per second, contained 45 lines and took about $1.8 s$ to complete. The characteristic time of concentration variation was about $2.5 \mathrm{~s}$. Therefore, although individual horizontal lines in Fig. 61B-E and vertical lines in Fig. 62 represent virtually instantaneous passive tracer distributions, the $2 \mathrm{D}$ concentration diagrams in Figs. 61B-E and 62 should not be considered as snapshots and are shown 
(A)

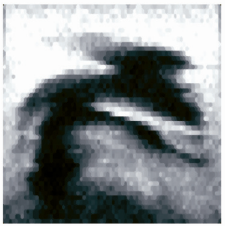

(D)

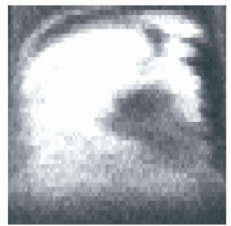

(G)

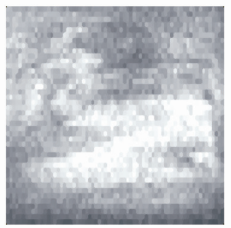

(B)

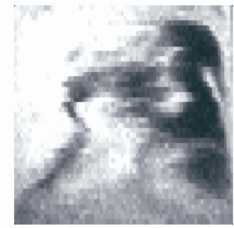

(E)

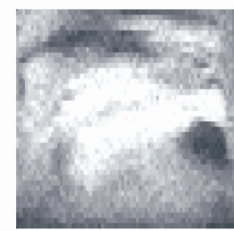

(H)

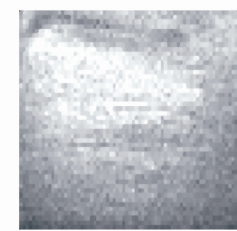

(C)

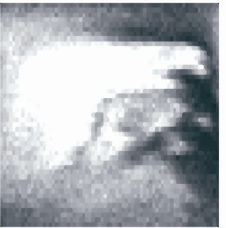

(F)

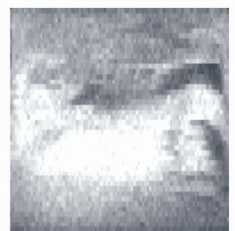

Figure 62. Distribution of tracer concentration in the vertical cross-section of the micro-channel at different locations downstream: (A) $\mathrm{N}=5$ (B) $\mathrm{N}=7$ $(\mathrm{C}) \mathrm{N}=9(\mathrm{D}) \mathrm{N}=11(\mathrm{E}) \mathrm{N}=13(\mathrm{~F}) \mathrm{N}=15(\mathrm{G}) \mathrm{N}=17(\mathrm{H}) \mathrm{N}=25$.

only for purpose of illustration. However, none of these diagrams was used for any quantitative measurements.

Corresponding to each value of $P e$ tested, mixing efficiency has been investigated by focusing on the long time statistics of the concentration distribution across the micro-channel at different positions, $N$, downstream. Typical time averaged concentration distributions across the micro-channel, $\bar{c}$, are displayed in Fig. 63. One can see that the concentration distribution close to the inlet, at $N=7$, is strogly influenced by the asymmetric conditions at the channel entrance. As one can learn from the curve corresponding to $N=11$, however, the imprint of the initial conditions is clearly fading out as the liquid is advancing downstream and the stirring becomes increasingly efficient. Further downstream, at $N=41$, asymmetry in the tracer distribution introduced by the initial conditions disappears completely.

Fading of the initial condition influence with time and restoration of 


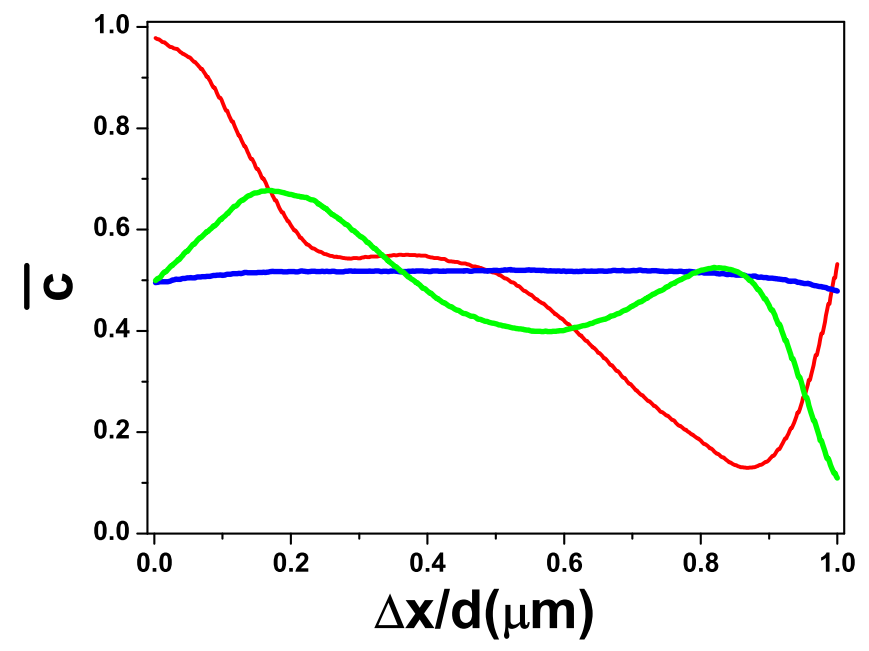

Figure 63. Time average of FITCD concentration, $\bar{c}$, as a function of the normalized coordinate across the micro-channel at different locations downstream: red- $N=7$, green- $N=11$ and blue- $\mathrm{N}=41$.

symmetry in flow in the statistical sense are both distinct features of chaotic and turbulent flows. Therefore, the curves displayed in Fig. 63 provide further evidence for truly chaotic nature of the microscopic flow. Variation of tracer concentration profiles with time at different distances from the inlet is illustrated by the space-time plots displayed in Fig. 64. One can see that the tracer concentration appears to fluctuate quite randomly in time. Next, as one can see in Fig. 64.A, which refers to $N=5$, that the left side of the channel, where the tracer was initially injected, looks much brighter and has a higher average concentration of the tracer. Although also noticeable in Fig.64.B taken further downstream at $N=18$, this feature is clearly weaker here.

A quantitative measure of the mixing efficiency is given by the $i$ th order moments, $M_{i}$, of a probability distribution function (PDF) of FITCD concentration, c, defined by $M_{i}=\frac{\left\langle|c-\bar{c}|^{i}\right\rangle}{\bar{c}^{i}}$. Statistics of concentration has been carried out over about $6 \times 10^{6}$ points, that is over $10^{4}$ individual concentration profiles across the micro-channel (Fig. 64). In Fig. 65 are presented the dependencies of $M_{1}$ and $M_{2}$ on the position along channel, $N$, corresponding to the largest value of $P e$ we investigated. As shown in Fig. 65, both 

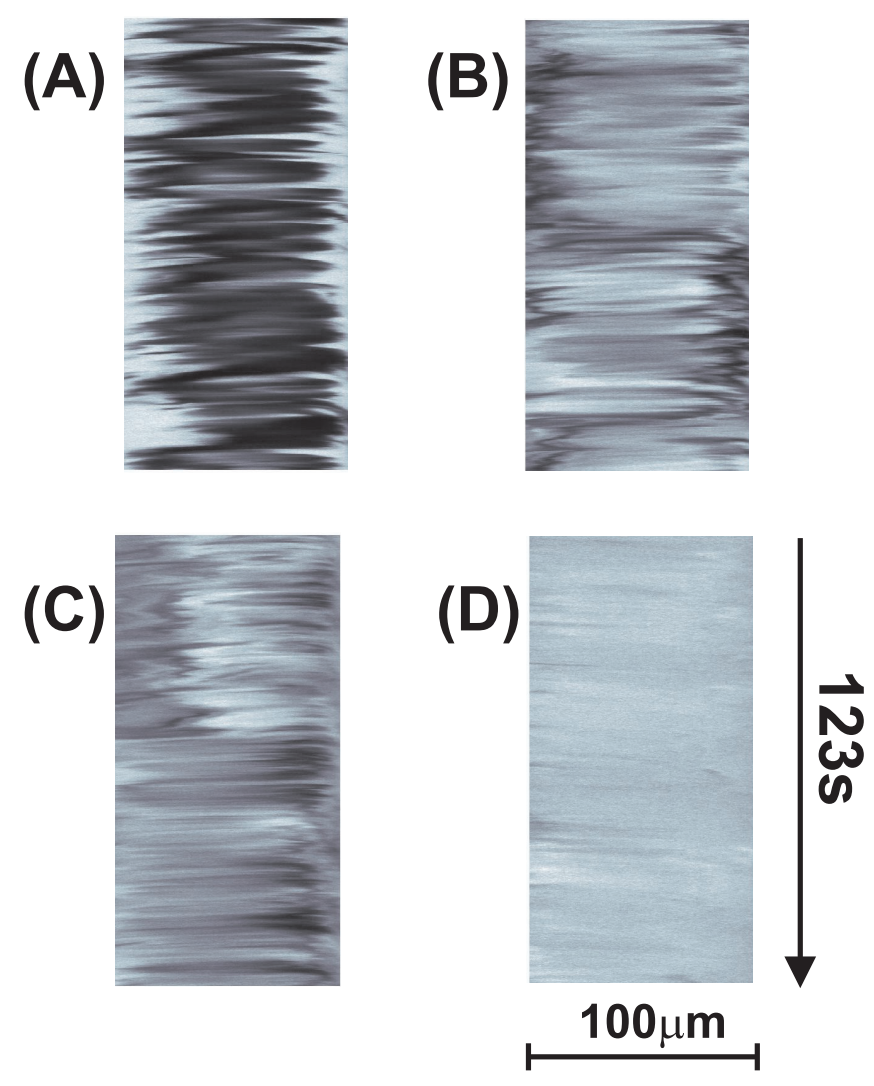

Figure 64. Space-time plots of FITCD distribution across the channel at different locations downstream: (A) $\mathrm{N}=5(\mathrm{~B}) \mathrm{N}=13$ (C) $\mathrm{N}=21$ (D) $\mathrm{N}=35$. Confocal scanning was done along the same line across the channel in the midplane at equal distances from the half-ring interconnections, with even time intervals of $0.0177 \mathrm{~s}$. 


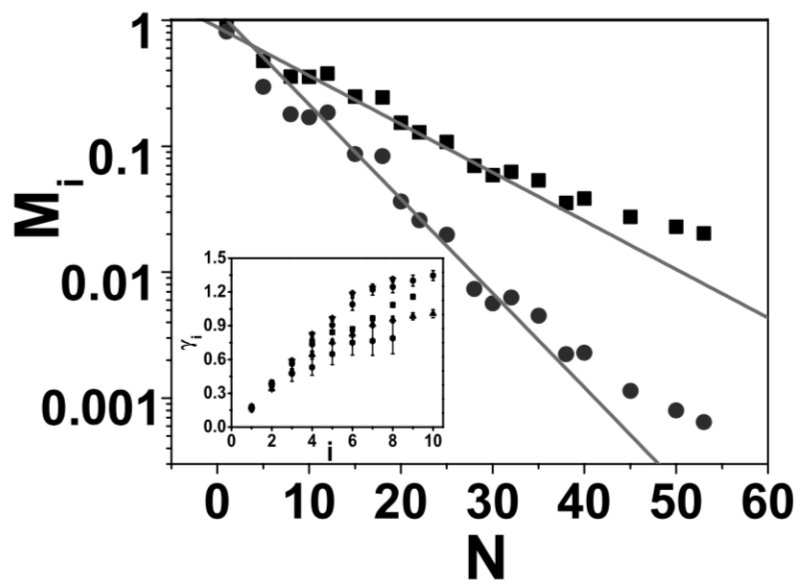

Figure 65. The first (squares) and second(circles) order moments decay vs. position, $\mathrm{N}$, at $P e=4.15 \times 10^{5}$; solid lines are the fit. Inset: the decay exponents of the moments, $\gamma_{i}$, as a function of the moment order, i, at different Pe: diamonds, $P e=1.6 \times 10^{4}$; up triangles, $P e=3.3 \times 10^{4}$; squares, $P e=9.09 \times 10^{4}$; circles, $P e=1.74 \times 10^{5}$; down triangles, $P e=4.15 \times 10^{5}$.

moments exhibit exponential decay up to $N \simeq 40$ as predicted by theory Son [1999], Chertkov and Lebedev [2003].

Second, the rate of decay of $M_{2}$ is twice as high as that of $M_{1}$. And third, $M_{1}$ is reduced more than 30 times and reaches the noise level at $N>40$, as well as $M_{2}$. In the inset of Fig. 65 are shown the decay exponents of the higher order moments, $\gamma_{i}$, as a function of their order, i, for each value of $P e$. One can notice that the saturation occurs for all curves at about the same value of $i=6$, but the saturated value of the decay exponents depends on $P e$.

The mixing length, measured in the number of units, $N_{m i x}$, and obtained from the exponential rate of decay of $M_{2}$, is presented in Fig. 66 as a function of $P e$. The data are well fitted by $N_{m i x} \propto P e^{0.26 \pm 0.01}$, which is in good agreement with theory Chertkov and Lebedev [2003].

The latter results indicates that the efficiency of the chaotic mixing is reduced due to finite size effects, which increase the mixing length in an bounded system compared to that in an unbounded one. 


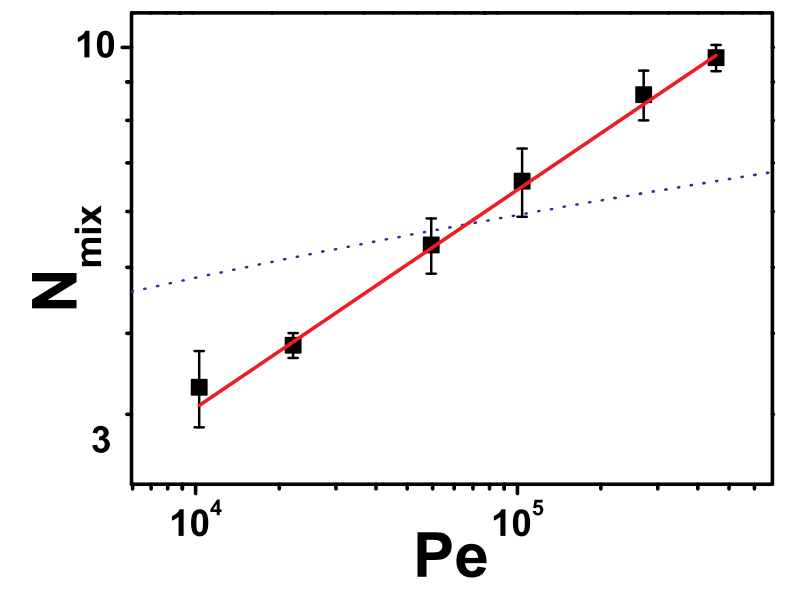

Figure 66. The mixing length, measured in the number of turns, $N_{m i x}$, vs. $P e$ : squares are the data, the solid line is the fit, and the dotted line shows the logarithmic dependence.

\subsection{Analysis of the mixing boundary layer}

In order to establish the source of the algebraic dependence of the mixing length instead of logarithmic, as predicted for an unbounded system, we have studied the $P e$ dependence of the mixing boundary layer width, $W$. The latter was identified from the spatial distribution across the microchannel of the first and second order moments of the probability distribution function of FITCD concentration fluctuations(Fig. 67).

We have associated well defined peaks in a spatial dependence of the moments with the boundary layer edge (note in Fig. 67 the asymmetry in the left and the right peak locations due to the flow asymmetry). The average value of the right peak location for the first moment (averaged along the micro-channel) as a function of $P e$ is presented in the inset of Fig. 67 together with the fit: $W \propto P e^{-0.28 \pm 0.06}$. This result is once more in fair agreement with the theoretical prediction $W^{-\frac{1}{4}}$ Chertkov and Lebedev [2003]. 


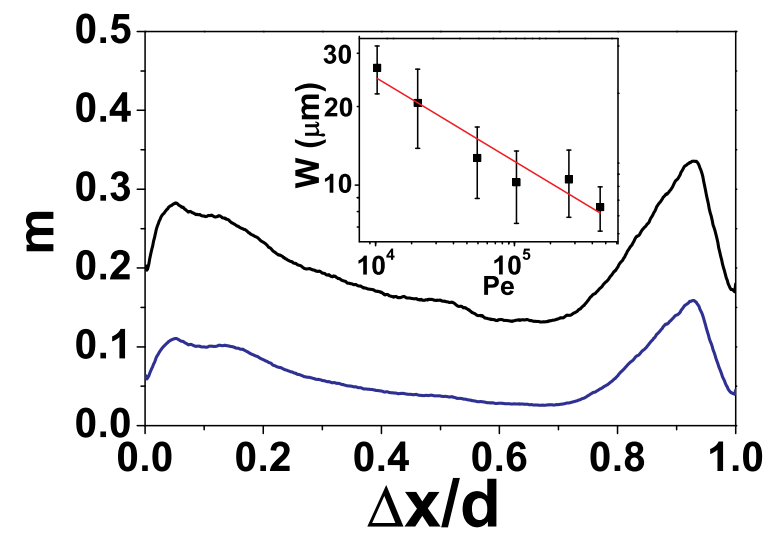

Figure 67. Spatial dependence of the first (blue line) and second (black line) order moments of FITCD concentration fluctuations across microchannel. Inset: width of the boundary layer, $W$, vs. $P e$. The solid line is the fit.

\subsection{Spatial and temporal correlations of the passive scalar fluc- tuations}

The spatial correlation across the micro-channel and temporal correlation functions of passive scalar concentration fluctuations in the bulk and in the boundary layer were studied at different $P e$. The spatial correlation functions decay logarithmically at distances above the diffusion length (which was varied from 1 to $4 \mu \mathrm{m}$ ) and are almost independent on $\mathrm{Pe}$ (Fig. $68 \mathrm{~A}$ ). The relatively narrow spatial range of the correlation function decay does not allow one to reliably distinguish between the logarithmic and power law behavior, if the exponent is small, as predicted by theory Chertkov and Lebedev [2003] (the exponent $\sim P e^{-\frac{1}{4}}$ ). This logarithmic like decay can be explained by the fact that the mixing in the bulk occurs rather efficiently. The mixing boundary layer permanently supplies the passive scalar into the bulk which mimics the Batchelor stationary regime of mixing Batchelor [1959a] and leads to logarithmic decay.

The temporal correlation functions in the bulk and in the boundary layer (Fig. 68 B,C) show similar behaviour with about twice as large correlation 

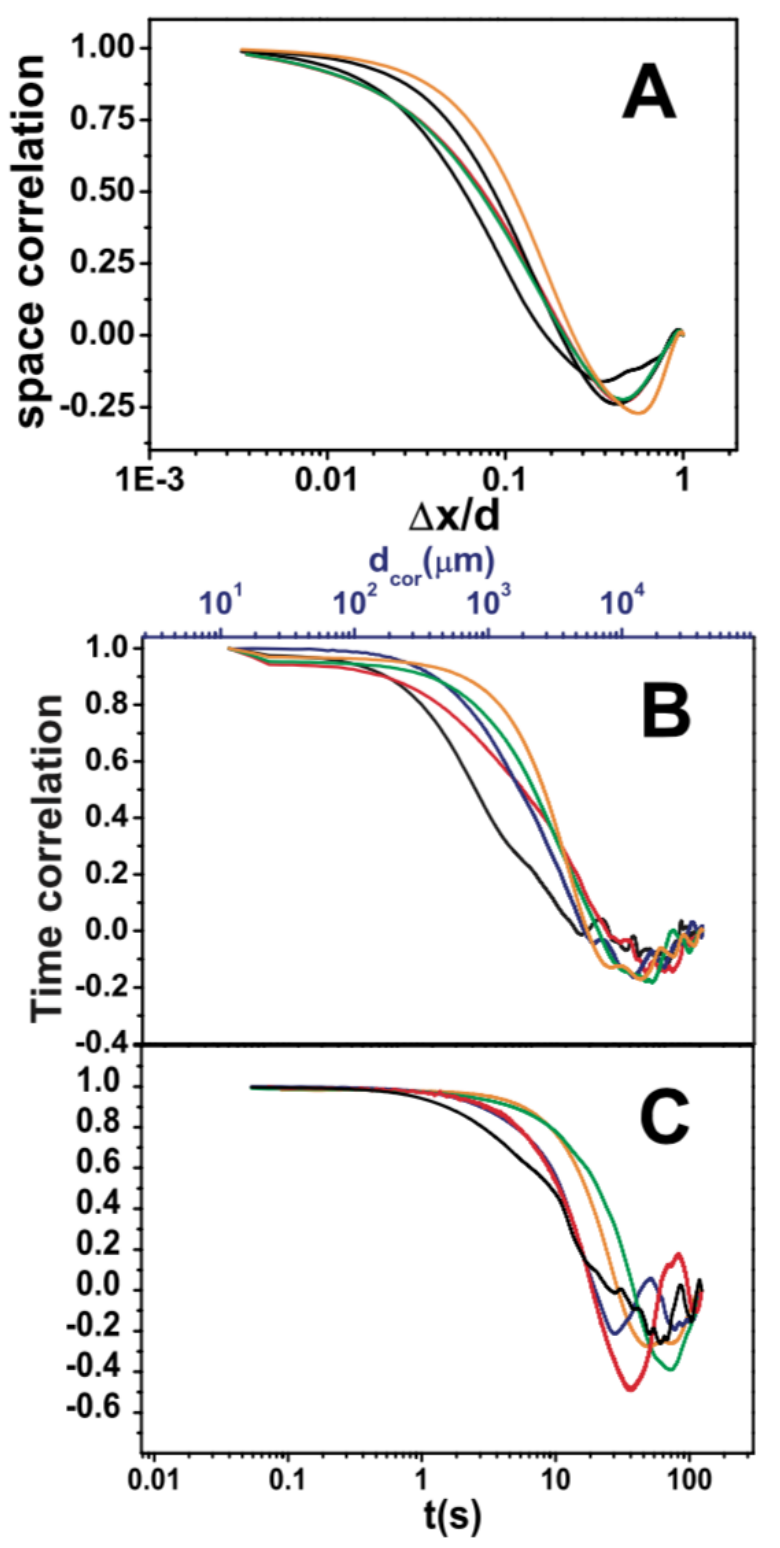

Figure 68. (A) Correlation coefficients for the tracer concentration vs. $\Delta x / d$. (B) Correlation coefficients for the tracer concentration vs. $\mathrm{t}$ in the bulk of the microdcopic flow. (C) Time auto correlation functions for the tracer concentration vs. near the boundary of the microscopic flow. The colours are: black- $P e=4.15 \times 10^{5}$, red- $P e=1.74 \times 10^{5}$, blue- $P e=$ $9.09 \times 10^{4}$, green- $P e=3.37 \times 10^{4}$, orange- $P e=1.68 \times 10^{4}$. 
time in the boundary layer as in the bulk $\left(t_{\frac{1}{2}} \approx 13 \mathrm{~s}\right.$ instead of $6 \mathrm{~s}$ in the bulk).

\section{Macroscopic heat transport by Elastic Turbulence}

The efficient transport of mass and heat within fluids is of paramount importance in many industrial settings such as the develop- ment of efficient heat exchangers, cooling the magnetic coils of particle accelerators and the central processing units (CPU's) of personal computers. The natural mechanisms by which the mass and the heat are transported within a fluid are the molecular diffusion and the thermal conduction, respectively. These mechanisms, however, are the least efficient ones in the sense that the characteristic times over which they take place are significantly large. An increase in the efficiency of the mass and heat transport can be obtained by generating flows with divergent Lagrangian trajectories which are able to efficiently stretch, fold and breakup the fluid elements down to sufficiently small spatial scales when the molecular diffusion and the thermal conduction can effectively homogenise the mass/temperatures distributions. One way of generating random fluid motion is to trigger inertial turbulent flow states by ensuring sufficiently large values of the Reynolds number $R e=\rho U L / \eta$. Here $\rho$ stands for the density of the fluid, $L$ for the characteristic size of the flow container, $U$ for the scale of the fluid velocity and $\eta$ for the viscosity of the fluid.

It has been demonstrated that inertial turbulent flows transport efficiently both mass and heat Gollub et al. [1991a], Lane et al. [1993], Warhaft [2000], Shraiman and Siggia [2000], Lee and Hyun [1999], Kim and Hyun [1997a]. There exist, however, various practical situations when increasing the Reynolds number is a difficult task, particularly when the characteristic size of the fluid container $\mathrm{L}$ is small, e.g. in a micro channel. In such situations, it is desirable to replace the inertial nonlinearity in the momentum equation with other types of nonlinearities. Exponentially divergent Lagrangian fluid trajectories can be generated by the laminar chaotic advection Aref [1990], Ottino [1989] and, consequently, such flows are able to efficiently transport both mass and heat Toussaint et al. [1995, 2000], Hobbs and Muzzio [1997], El Omari and Le Guer [2010], Acharya et al. [1992], Mota et al. [2007]. Triggering the laminar chaotic advection at moderate Re requires, however, a special design of the flow channel and/or a particular flow control, e.g. controlled pulsations. This can also become quite challenging in the case of microscopic flows due to technical limitations/difficulties in manufacturing elaborated three dimensional microstructures. 


\subsection{Experimental setup and methods}

The experimental setup is schematically illustrated in Fig. 69. It consists of an acrylic made fluid container $\mathbf{F C}$ with the radius $R_{c}=40 \mathrm{~mm}$ and optically transparent walls. The flow is driven by a rotating top disk $\mathbf{D}$ with a radius $R_{d}=39 \mathrm{~mm}$ mounted on the shaft of a commercial rheometer, Mars III (from Thermofischer). The use of the commercial rheometer in driving the flow is justified by the accurate control of both the rotation speed $\Omega$ and the driving torque $M$. The distance between the top disk and the bottom of the fluid container was $H=60 \mathrm{~mm}$ for all the experiments reported in the paper.

The temperature of the bottom of the fluid container is controlled via a circulating fluid bath CFB fed with de-ionised water by a thermally stabilised fluid circulator (Lauda, model Proline RP 855). To avoid triggering the thermal convection, the temperature within the circulating fluid bath CFB is set to $T_{b}=13^{\circ} \mathrm{C}$ which is smaller than the room temperature $T_{0}=23^{\circ} \mathrm{C}$. To ensure a good repeatability and reproducibility of the experiments, the room temperature has been regulated (with an accuracy of $\pm 0.5^{\circ} \mathrm{C}$ ) at all times and for each of the experiments reported herein by an air conditioning system installed in the experimental room.

The distribution of the temperature into the flow is point-wise monitored by an array of six thermocouples $T_{1-6}$ (Chromel-Alumel, $100 \mu \mathrm{m}$ in size) disposed equidistantly along the vertical direction $z$ and positioned at the radial position $r=R_{c} / 2$, Fig. 69. The thermocouples are mounted through thin metal tubes which allows one to scan the temperature distribution along the radial direction. The signals of the thermocouples are passed to the digitising block $\mathbf{A}_{\mathbf{2}} \mathbf{D}$ via the reference box $\mathbf{R B}$. The subtraction of the reference temperature by the reference box $\mathbf{R B}$ diminishes the instrumental error of the temperature readings down to roughly $0.5 \%$ of the measured value.

Together with the point-wise measurements of the temperature, the flow has been investigated by local measurements of the flow fields by the Digital Partical Image Velocimetry (DPIV) technique. For this purpose, a green laser beam $(\lambda=514 \mathrm{~nm})$ with a power of $500 \mathrm{~mW}$ emitted by the solid state laser L (from Changchun Industries, Model LD-WL206) is deflected by a system of planar mirrors to a cylindrical optics block $\mathbf{C O}$ which reshapes it in a horizontal laser sheet LS, Fig. 69. The cylindrical optics block is composed of a glass rod with a short focal distance $f_{1}=2 \mathrm{~mm}$ and a cylindrical lens with a larger focal distance, $f_{2}=7 \mathrm{~cm}$. The two optical elements are mounted orthogonally to each other and in a telescopic arrangement such as the primary horizontal laser sheet generated by the glass rod is focused on the vertical direction in the middle of the flow channel by the second lens 


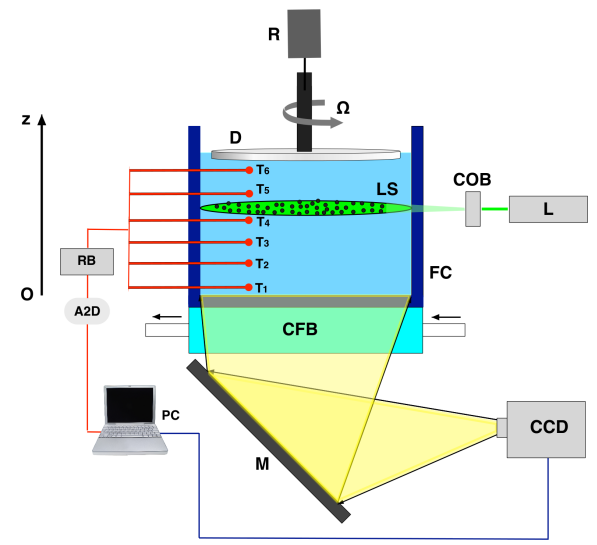

Figure 69. Schematic view of the experimental setup: $\mathbf{R}$ - the shaft of the rheometer, D - rotating disk, FC - fluid container, CFB - circulating fluid bath, $\mathbf{T}_{\mathbf{1}-\mathbf{6}}$ - thermocouples, $\mathbf{R B}$ - reference box, $\mathbf{A}_{\mathbf{2}} \mathbf{D}$ - analogue to digital converter , L - solid state laser, LS - laser sheet, $\mathbf{C O B}$ - cylindrical optics block, $\mathbf{M}$ - planar mirror, CCD - video camera, PC - personal computer.

minimising thus its thickness in the measurement region. The thickness of the generated laser sheet is roughly $80 \mu \mathrm{m}$ in the beam waist region which is positioned at the centre of the fluid container FC. The horizontal laser sheet has been positioned in the vicinity of the thermocouple $T_{4}$, Fig. 69 . The working fluid was seeded with an amount of 200 parts per million ( $\mathrm{ppm}$ ) of polyamide particles with a diameter of $20 \mu m$ (from Dantec Dynamics).

Time series of the velocity fields were obtained by a iterative multi-grid DPIV algorithm implemented in house under Matlab Raffel et al. [September 2007], Scarano and Rhiethmuller [2001]. For this purpose, a sequence of flow images has been acquired with a colour CCD camera which visualises the illuminated flow section through the planar mirror $\mathbf{M}$. The total image acquisition time was one minute within the laminar regime and five minutes within the transitional and fully developed elastic turbulent regimes which allowed one to properly capture the flow de-correlation beyond the onset of the primary elastic instability. The inter-frame has been adapted to the measured flow speed in order to maintain the average displacement of the tracer particles in the optimal range of $5-15$ pixels. Together with this, the size of the smallest interrogation window has been adapted to the mean flow velocity corresponding to the value angular speed of the top rotating 


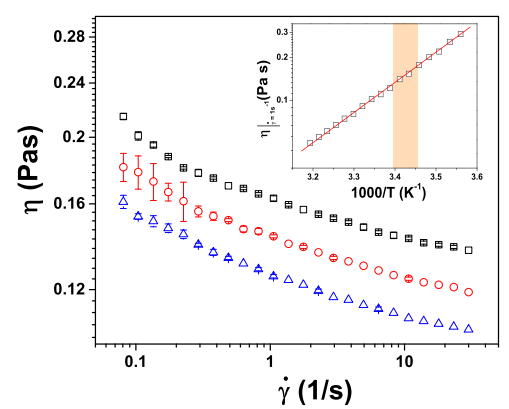

(a)

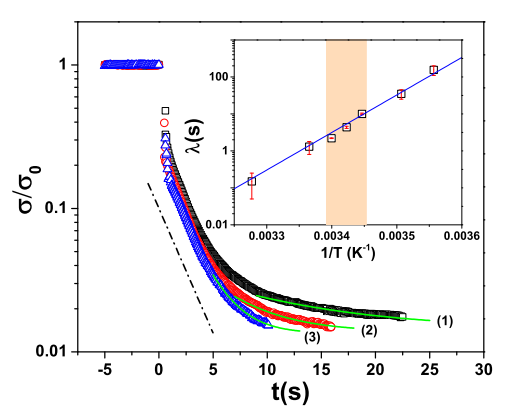

(b)

Figure 70. (a) Flow curves measured during controlled rate flow ramps. The insert presents the temperature dependence of the viscosity measured at $\dot{\gamma}=1 \mathrm{~s}^{-1}$ and the full line is an Arrhenius fit. (b) Stress relaxation data. The full lines are exponentially decaying functions characterising the slow relaxation mode. The dash-dot line is a guide for the eye, $\sigma / \sigma_{0} \propto e^{-t / 2.6( \pm 0.2)}$, highlighting the fast decay mode. The temperature dependence of the longest relaxation time is presented in the insert. The error bars are defined via the nonlinear fitting error. In each panel, the symbols refer to different operating temperatures: squares $(\square)-T=17^{\circ} C$, circles (o) $-T=19^{\circ} C$, triangles $(\triangle)-T=21^{\circ} C$.

disk $\mathbf{D}$ which sets a scale for the mean displacement of the flow tracers in the field of view. The spatial resolution of the measured flow fields was $3 \mathrm{~mm}$. Using this adaptive DPIV protocol the instrumental error of the measured velocity fields does not exceed $7 \%$ through all the experiments reported herein.

A 100 parts per million (ppm) anionic polyacrylamide (PAAM) solution with the molecular weight $M_{w}=22 \cdot 10^{6} \mathrm{Da}$ has been used as a working fluid. The solvent was a $65 \%(w t)$ aqueous solution of sucrose. The anhydrous sucrose was not of an analytical grade (pure) but simply a commercially available sugar (Sucre Crystal from Béghin Say, France). The viscosity of the Newtonian solvent was $\eta_{s}=114 \mathrm{mPas}$ (at the room temperature $T_{0}$ ) which ensures large characteristic relaxation times of the polymer solution.

The polymer solutions were prepared according to the following protocol. First we dissolved $0.9 \mathrm{~g}$ of anhydrous PAAM and $3 \mathrm{~g}$ of $\mathrm{NaCl}$ into $275 \mathrm{ml}$ of de-ionised water by gentle shaking. $\mathrm{NaCl}$ was added to fix the ionic contents within the polymer solution. Next the solution was mixed for $3 h$ in a commercial mixer with a propeller at a moderate speed. The rationale for this 
step is to cause a controlled mechanical degradation of the longest PAAM molecules, in order to cut the tail of the molecular weight distribution of the broadly dispersed PAAM sample. In a solution with a broad distribution of polymer molecular weights, the heaviest molecules, which are most vulnerable to mechanical degradation, bring the major contribution to the solution elasticity, but may break in the course of the experiment. This can lead to inconsistency of the experimental results. We found empirically that the procedure of pre-degradation in the mixer leads to substantial reduction of degradation during the experiments and to substantial improvement of their consistency. Finally, $9 g$ of isopropanol was added to the solution to preserve it from ageing and water was added up to $300 \mathrm{~g}$. The final concentrations of PAAM, $\mathrm{NaCl}$, and isopropanol in the stock solution were $3000 \mathrm{ppm}, 1 \%$, and $3 \%$, respectively. This master solution was used to prepare $100 \mathrm{ppm}$ PAAM solution in a Newtonian solvent. To prevent the bacterial growth and allow the storage over extended periods of time we have finally added to the polymer solutions an amount of $250 \mathrm{ppm}$ of sodium azide $\left(\mathrm{NaN}_{3}\right)$.

The density of the polymer solution was measured at room temperature by accurately weighting fixed volumes of fluid, $\rho=1200 \mathrm{kgm}^{-3}$.

The thermal properties of the polymer solution have been investigated using a hot disk thermal conductivity analyser. The Transient Plane Source (TPS), or the Hot-Disk method is a commonly used experimental technique for measuring thermal properties of materials in either a solid or fluid state proposed by Gustaffson in early 90's, Gustafsson [1991], Gustavsson et al. [1994].

The thermal diffusivity of the pure solvent was measured $\kappa_{s}=2.21$. $10^{-7} \mathrm{~m}^{2} \mathrm{~s}^{-1}$. The thermal diffusivity coefficient of the polymer solution was measured $\kappa=1.31 \cdot 10^{-7} \mathrm{~m}^{2} \mathrm{~s}^{-1}$ and the characteristic time associated to the thermal diffusion may be estimated as $t_{d}=H^{2} / \kappa \approx 25714 \mathrm{~s}$. We also note that within the temperature range explored through our experiments no significant temperature dependence of the thermal diffusivity coefficient was observed.

The rheological properties of the solvent and of the polymer solution were measured with the same Mars III rheometer around which the complete experimental setup was built. The shear viscosity of the solution was measured with a cone and plate geometry $(D=60 \mathrm{~mm}, 2$ deg truncation) via a controlled rate flow ramp at three temperatures which are relevant during the heat transfer process, $T=17^{\circ} \mathrm{C}, 19^{\circ} \mathrm{C}, 21^{\circ} \mathrm{C}$. Corresponding to each value of the imposed shear rate the stress was allowed to equilibrate during $50 \mathrm{~s}$ and the averaging was performed during the last $10 \mathrm{~s}$. The choice of this temperature range was based on the value of the room temperature and of the thermal equilibrium temperature achieved during our 
experiments, $T_{e} \approx 16.9^{\circ} \mathrm{C}$. The temperature was controlled during the rheological measurements by a Peltier system. The geometry has been enclosed by a teflon made solvent trap in order to minimise the sample evaporation and insure the reliability of the measurements. For each temperature, the viscosity measurement has been repeated three times with a freshly loaded sample in order to check the reliability and specify an error bar. The results of the shear viscosity measurements performed in a range of shear rates and temperatures relevant to the heat transport experiments are presented in Fig. 70(a). The viscosity follows an Arrhenius time dependence on the temperature (see the full line in the insert of Fig. $70(\mathrm{a})), \eta \propto e^{\frac{E_{a}^{\eta}}{R T}}$, where $R$ is the ideal gas constant. The viscosity activation energy is found $E_{a}^{\eta} \approx 40.625( \pm 1.2) k J /(\mathrm{mol} K)$. The largest polymer relaxation time has been assessed using the stress relaxation method, Liu et al. [2007, 2009]. For this purpose, a constant rate of shear $\dot{\gamma}=1 \mathrm{~s}^{-1}$ has been maintained for $50 \mathrm{~s}$. At the time instant $t_{0}=0$ the rate of shear has been set to 0 and the time decay of the stress has been monitored. As compared to the small amplitude oscillatory measurements, the relaxation time assessed via this technique is independent on the value of the initial shear (if the initial shear rate is sufficiently small so the inertial effects associated to both the shaft of the rheometer and the measuring geometry don't play a significant role), Liu et al. [2007]. In addition to that, the largest relaxation time obtained via this method is directly comparable to the relaxation time measured via the relaxation of single molecules observed via fluorescent microscopy, Liu et al. [2007]. Relaxation measurements performed at the same temperatures as the shear measurements are illustrated in Fig. 70(b). At each temperature a two mode exponential decay is observed. The fast mode highlighted by the dash-dotted line has a characteristic time $\lambda_{1}=2.6 s( \pm 0.2 s)$ which is independent on the operating temperature. The largest relaxation time $\lambda$ associated to the slow mode (see the full lines in Fig. 70(b) ) depends on the temperature according to an Arrhenius type law $\lambda \propto e^{\frac{E_{a}^{\lambda}}{R T}}$, the insert in Fig. 70(b) . Although it is commonly believed that the viscosity activation energy should be close to the relaxation time activation energy, we obtain a significantly larger value, $E_{a}^{\lambda} \approx 193.5( \pm 4) k J /(m o l K)$. A possible explanation for this rather unexpected result is that the changes of the relaxation time with the temperature for this polymer solution are triggered not only by the changes of the solvent viscosity but some other changes in the molecular properties of the polymer chains. By taking into account the shear thinning of the viscosity illustrated in Fig. 70(a), the Reynolds number was calculated according to $R e=\frac{\Omega R_{c}^{2} \rho}{\eta(\dot{\gamma})}$ where $\Omega$ is the angular speed of the top driving disk $\mathbf{D}$. Corresponding to the largest value of $W i$ we have 
explored and considering an average value of the shear viscosity within the relevant temperature range, we have found $R e \approx 26$. The main conclusion of the thermorheological analysis presented above is that in spite of a rather limited temperature range non-negligible changes in both the shear viscosity and the largest polymer relaxation time are observed. Thus, even in the absence of a buoyancy term in the momentum equation (in which case the temperature acts as an active scalar field), one can not a priori establish a similarity between the dynamics of a passive scalar in a random smooth flow and the transport of heat by elastic turbulence. This further reinforces the motivation of our study. The Prandtl number $\operatorname{Pr}=\frac{\eta(\dot{\gamma})}{\rho \kappa}$ which quantifies the balance between the viscous momentum diffusion and the thermal diffusion varied during our experiments (because of the shear thinning behaviour of the viscosity and its $T$ dependence) in the range $\operatorname{Pr} \in[744 ; 1336]$. The balance between natural and forced convection is quantified by the Richardson number which may be defined as $R i=\frac{g \beta \Delta T}{R_{c} \Omega^{2}} h^{3}$ where $g$ is the acceleration of gravity, $\beta \approx 2 \cdot 10^{-4} K^{-1}$ is the thermal expansion coefficient, $\Delta T=T_{0}-T_{b}$ and $h=H / R_{c}$ is the geometric aspect ratio of the flow cell. By varying the angular speed of the top disk, the Richardson number was varied during our experiments in the range $R i \in[4.7 ; 48.8]$.

\subsection{Observation and characterisation of elastic turbulent flow states}

We focus in the following on a brief characterisation of the transition to elastic turbulence and its main flow features in isothermal conditions at the room temperature $T_{0}$. At an integral scale, the transition to elastic turbulence via a primary elastic instability can be monitored by measurements of the statistics power $P$ injected into the flow as a function of the Weissenberg number. The injected power is calculated for the case of a swirling flow as $P=M \cdot \Omega$ where $M$ is the torque acting on the shaft of the rheometer.

The statistics of the power fluctuations measured with the polymer solution as a function of the Weissenberg number is illustrated in Fig. 71. The Weissenberg number was calculated as $W i=\dot{\gamma} \cdot \lambda$. Based on the measurements presented in Fig. 70(b), we have chosen $\lambda=5.5 \mathrm{~s}$ which is the average value within the temperature interval considered. Corresponding to a a critical value of the Weissenberg number $W i_{c} \approx 2$, an increase of the flow resistance is observed in the form of an increase of the averaged injected power beyond its laminar value $\bar{P}_{l a m}$, Fig. 71(a). This corresponds to a primary elastic instability that occurs in the flow. Simultaneously with this, an increase of the rms of the power fluctuations is observed, Fig. 71(b). The experimentally determined scaling laws of both the flow 

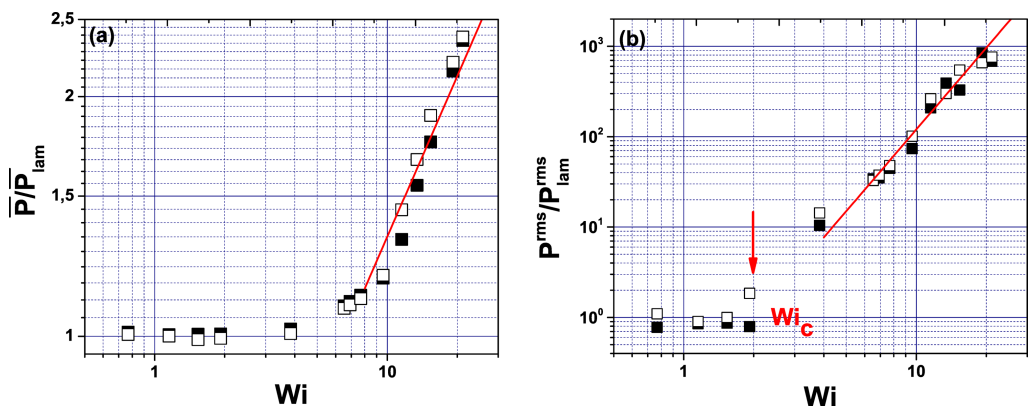

Figure 71. (a) Dependence of the normalised time averaged injected power $\bar{P} / \bar{P}_{\text {lam }}$ on the Weissenberg number $W i$. The full line is a guide for the eye, $\bar{P} / \bar{P}_{\text {lam }} \propto W i^{0.67}$. (b) Dependence of the reduced rms of the power fluctuations $P^{r m s} / P_{l a m}^{r m s}$ on the Weissenberg number $W i$. The full line is a guide for the eye, $P^{r m s} / P_{l a m}^{r m s} \propto W i^{3}$. The full/empty symbols refer to increasing/decreasing $W i$. The vertical arrow indicates the onset of the primary elastic instability $W i_{c}$.

resistance $\bar{P} / \bar{P}_{\text {lam }} \propto W i^{0.67}$ and of the reduced rms of the fluctuations, $P^{r m s} / P_{\text {lam }}^{r m s} \propto W i^{3}$, are consistent with previous measurements, Burghelea et al. [2007], Burghelea [2005].

The evolution of the flow structure with the Weissenberg number is illustrated in Fig. 72. The DPIV measurements are performed in an horizontal plane located in the vicinity of the thermocouple $T_{4}$ at $z \approx H / 2$ (Fig. 69). The top row of Fig. 72 presents the flow fields averaged over times typically 10 times larger than the polymer relaxation time $\lambda$ and the bottom row presents the time averaged in plane vorticity $\left\langle\omega_{z}\right\rangle_{t}$.

The flow field measured below the onset of the primary elastic instability at $W i=0.8$ is dominated by a stationary and off-centred Ekman vortex, Ekman [1905]. This flow structure is different from that of the laminar flow observed in Ref. Burghelea et al. [2007] (see Fig. 10(a) therein) which was consistent with a rigid body rotation around the symmetry axis of the fluid container FC and had no vertical velocity component. This difference can be explained by the presence of the array of thermocouples which perturb the base flow, see Fig. 69. The off-centred flow topology observed in the laminar regime is consistent with the presence of a steady vertical flow component which may slightly enhance the heat transfer (as compared to the purely conductive state) even below the onset of the elastic instability. The effect of the thermocouples on the laminar flow can also be noticed in the map of the time averaged vorticity presented on the second row of 


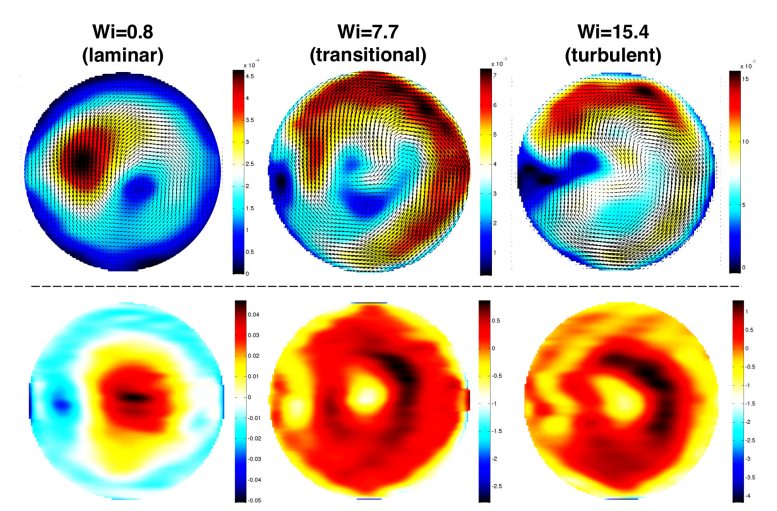

Figure 72. Top row: time averaged flow patterns for various Weissenberg numbers indicated in the top inserts. The colour map refers to the modulus of the in-plane velocity. For the clarity of the presentation, the overlapped vector fields were down-sampled by a factor of 2 along each direction. Bottom row: time averaged vorticity, $\left\langle\omega_{z}\right\rangle_{t}$. The measurements were performed in isothermal conditions at the room temperature $T_{0}$.

Fig. 72. The topology of the mean flow and vorticity field changes as one moves towards the transitional $(W i=7.7)$ and fully developed elastic turbulent $(W i=15.4)$ flow regimes. Within the transitional regime, the flow is dominated by an unsteady toroidal vortex. Within the elastic turbulent regime, the flow topology changes to a randomly fluctuating spiral vortex, in agreement with the previous results on the elastic turbulence in von Karman flows, Burghelea et al. [2007], Burghelea [2005]. Within the elastic turbulent regime the flow is spatially smooth, strongly correlated over space and its characteristic correlation length is comparable to the radius $R_{c}$ of the fluid container FC, Burghelea et al. [2005, 2004b]. The main flow illustrated above are consistent with previous experimental results Burghelea et al. [2007], Burghelea [2005] and recommend the elastic turbulence as a potential candidate to efficiently transport the heat within the von Karman flow.

\subsection{Heat transport by Elastic Turbulence}

Prior to investigating the heat transfer process in a regime of elastic turbulence, we focus on the heat transfer within the flow of the sucrose solvent at $R e \approx 125$ which is significantly larger than the largest $R e$ number investigated during the experiments with the polymer solution. Transient measurements of the reduced temperature $\theta=\frac{T_{0}-T}{T_{0}-T_{b}}$ performed at various 

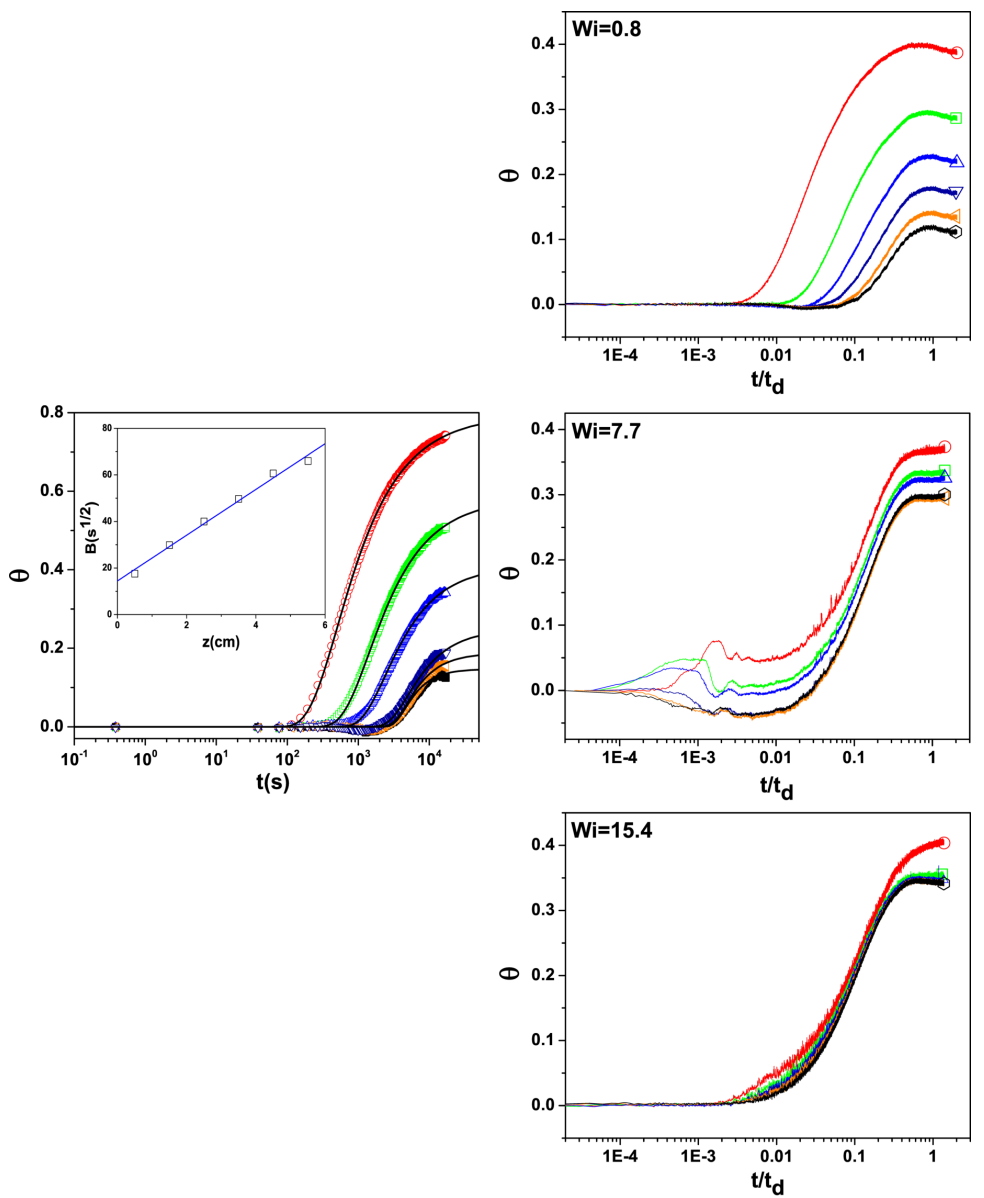

(a)

(b)

Figure 73. (a) Time series of the reduced temperature measured with the sucrose solvent at $R e \approx 125$ : circle $(\circ)-T_{1}$, square $(\square)-T_{2}$, up triangle $(\triangle)-T_{3}$, down triangle $(\nabla)-T_{4}$, left triangle $(\triangleleft)-T_{5}$, hexagon $(\square)$ - $T_{6}$. All measurements were performed at a distance $r=R_{c} / 2$ from the symmetry axis of the flow container FC. The full lines are nonlinear fitting functions (see explanation in the text). The dependence of the fit parameter $B$ (see text for explanation) on the vertical coordinate is presented in the insert.(b) Time series of the reduced temperature measured at three Weissenberg numbers indicated in the inserts of each panel. The symbols in each panel refer to the vertical position of the thermocouple (see Fig. 69): circle (०) - $T_{1}$, square $(\square)-T_{2}$, up triangle $(\triangle)-T_{3}$, down triangle $(\nabla)-T_{4}$, left triangle $(\triangleleft)-T_{5}$, hexagon $(\square)-T_{6}$. All measurements were performed at a distance $r=R_{c} / 2$ from the symmetry axis of the flow container FC.. 
vertical positions $z$ and $r=R_{c} / 2$ are presented in Fig. 73(a). Each transient data set may be formally ${ }^{12}$ fitted by $\theta=A \cdot \operatorname{erfc}\left(\frac{B}{\sqrt{t}}\right)^{C}$ (the full lines in Fig. 73(a)) where erfc is the complementary error function, the parameter $A$ describes the equilibrium temperature and $B=\frac{z}{(4 \kappa)^{1 / 2}}$ describes the local intensity of the heat transfer process. By fitting linearly the values obtained for the coefficient $B$ against the positions $z$ of the thermocouples (see the insert in Fig. 73(a)) one obtains for the thermal diffusivity of the solvent $\kappa_{s} \approx 2.6 \cdot 10^{-7} \mathrm{~m}^{2} \mathrm{~s}^{-1}\left( \pm 2.3 \cdot 10^{-8} \mathrm{~m}^{2} \mathrm{~s}^{-1}\right)$ which is fairly close to the measured value. The equilibrium reduced temperatures reached by each thermocouple are strongly dependent on the vertical coordinate $z$ indicating that no efficient mixing occurs in the flow which is consistent with the laminar and linear flow behaviour of the solvent alone. In addition to that, no fluctuations of the reduced temperature can be noted in Fig. 73(a) which is once more consistent with a laminar flow behaviour. To conclude, the tests performed with the Newtonian sucrose solvent alone at the largest Reynolds number explored through the experiments with the polymer solution revealed a conductive like heat transport mechanism characterised by a strong spatial dependence of the equilibrium temperatures and a lack of temperature fluctuations. We now turn our attention to the case when the polymer solution is used and the Elastic Turbulence is triggered.

Measurements of the time series of the reduced temperature $\theta$ performed within each flow regime are presented in Fig. 73(b). The horizontal axis is normalised by the characteristic diffusion time $t_{d}$. Due to the presence of the secondary flow, one can no longer resort to the complementary error function nonlinear fit used in the case of the pure solvent illustrated in Fig. 73(a). On the other hand it can be noted that, regardless the value of the Weissenberg number and the measuring position, each reduced temperature time series exhibits a logarithmic scaling part (note that the data is plotted in lin-log coordinates) $\theta \propto a+b \cdot \ln \left(t / t_{d}\right)$ before reaching a steady state plateau corresponding to $t / t_{d} \approx 1$.

The six data sets acquired within the laminar regime at $W i=0.8$ never collapse indicating that a temperature gradient along the vertical direction $z$ exists at all times, top panel in Fig. 73(b). Secondly, the local slope $b$ of the log scaling part of the reduced temperature series decreases monotonically from the bottom plate (the data set marked by a circle) to the top plate of the fluid container. This indicates that the intensity of the heat transfer

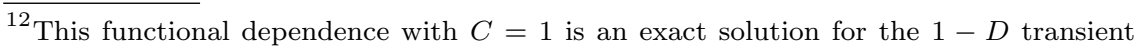
heat transfer problem only in the case of a semi-infinite planar domain with a constant temperature boundary condition. Deviations from the analytically exact result related to finite size effects may be accounted for by letting $C$ vary as an extra fit parameter.
} 
is strongly inhomogeneous along the vertical direction and it is the highest near the heat sink (the bottom plate) which is what one would expect in the case of a conduction dominated heat transfer regime. Prior to entering the logarithmic scaling regime, the time series of the reduced temperature pass through a local minimum $\theta^{\text {min }}<0$. The magnitude of this minimum decreases as one moves from the top part of the fluid container to its cooled bottom and it practically disappears at the level of the thermocouple $T_{1}$. We interpret this effect in terms of local viscous heating of the polymer solution.

The reduced temperature time series acquired within the transitional regime $(W i=7.7)$ are presented in middle panel in Fig. 73(b). As compared to the laminar case, several differences may be noted. First, within the logarithmic scaling range, the separation between the measurements performed at the six vertical positions is much smaller and so are the differences in the plateaus observed around $t \approx t_{d}$. This is an indication that the unsteady vortical flow observed within the transitional regime (see Fig. 72) homogenises the vertical distribution of the temperature more efficiently than the steady laminar vortex. Second, one can clearly observe in the middle panel of Fig. 73(b) a fluctuating component of the temperature signal which is most pronounced near the bottom plate of the flow container (the data set labeled by a circle). Third, the local minimum of the temperature series acquired near the top plate is more pronounced than in the laminar case and occurs earlier which corroborates with our interpretation in terms of local viscous heating.

The six temperature time series acquired within the fully developed elastic turbulent regime at $W i=15.4$ nearly collapse onto a single master curve which indicates a perfectly homogeneous distribution of the temperature within the system, bottom panel of Fig. 73(b). Together with this, an increased level of fluctuations is observed within the logarithmic scaling range. No local minimum related to viscous heating is observed which can be explained by an efficient transport of the heat from the top of the flow cell (note the viscous heating is more effective near the top driving disk $\mathbf{D}$ due to the inhomogeneous shear in a von Karman flow configuration) to the colder bottom via the randomly fluctuating spiral vortex. For a quantitative assessment of the local intensity of the heat transfer at various Weissenberg numbers we resort to the logarithmic slope $b$ introduced above. The dependence of the intensity factor $b$ obtained from the data acquired at various vertical positions on the Weissenberg number $W i$ is presented in Fig. 74 .

Because the base flow does have a non-zero vertical component, an increase of the local intensity of the heat transfer related to the increase of the in plane vorticity is observed even below the onset of the primary elastic 


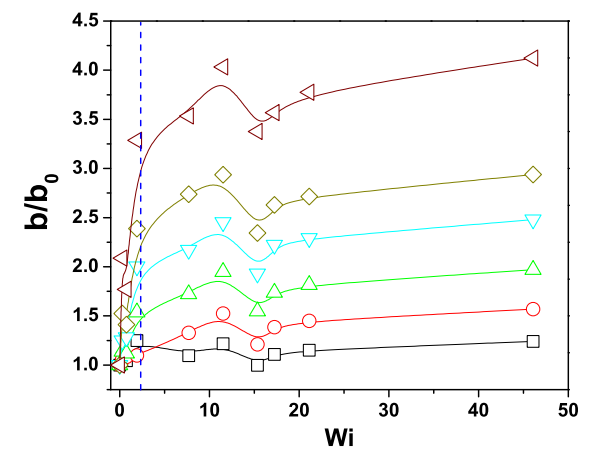

Figure 74. Dependence of the efficiency factor $b$ obtained at various vertical positions indicated in the insert on the Weissenberg number. The symbols refer to the position along the vertical axis $z$ (see Fig. 69): ( $\square$ )- $T_{1}$, (०)- $T_{2}$, $(\triangle)-T_{3},(\nabla)-T_{4},(\diamond)-T_{5},(\triangleleft)-T_{6}$. The vertical dashed line indicates the onset of the primary elastic instability $W i_{c}$.

instability, $W i=W i_{c}$.

As compared to the purely conductive case, the transition to the elastic turbulence is accompanied by a roughly $3.5-4$ fold increase of the local intensity of the heat transfer at the position of the thermocouples $T_{4}-T_{6}$. At large $W i$ the intensity reaches a plateau which is related to the plateau of the mean flow vorticity.

We also note that an increase of the local intensity of the heat transfer is observed in the vicinity of the top driving disk slightly below the onset of the primary elastic instability. This is due to the presence of the laminar Ekman vortex induced by the intrusive presence of the thermocouples and pictured in Fig. 72 (first column, top row). Unfortunately, this effect can be neither removed nor decoupled from the elastic turbulence contribution and this is a clear drawback of our experimental technique.

It is interesting to compare the increase in the intensity of the heat transport by elastic turbulence with similar experiments performed with Newtonian fluids at large $R e$ in a regime of inertial turbulence. In Ref. Gollub et al. [1991b] Gollub and his coworkers asses the efficiency of the heat transfer via the effective thermal diffusivity $D^{*}=K^{*} / \rho c_{p}$ where the global heat transport coefficient $K^{*}$ is obtained from the ratio of the total measured heat flux and the local temperature gradient, $\rho$ is the density of the fluid, and $c_{p}$ is its the heat capacity. As illustrated in Fig. 2 of their paper, an increase of the efficiency of up to a factor of ten is observed at $R e=6000$. 
The local increase in the heat transport intensity of roughly $3.5-4$ times we have measured within a regime elastic turbulence at $W i=15.4$ corresponds to the increase observed in Ref. Gollub et al. [1991b] at $R e \approx 1600$. It is equally interesting to compare our results with numerical simulations performed for the case of a von Karman flow of a Newtonian fluid. Prior to discussing this, we note however that a rigorous and systematic comparison of the increase of the heat transfer efficiency by elastic turbulence with the Newtonian counterpart in a swirling flow is not possible for several reasons. First, unlike in numerical simulations, we are unable to measure the Nusselt number and we have provided only a local measure of the intensity of the transfer by the coefficient $b$. Secondly, during our experiments, when the Weissenberg number is increased, both the Richardson and the Prandtl numbers are varied and their range does not necessarily match that studied in numerical simulations (both numbers are larger in our case). Last but not least, the flow structure within a regime of elastic turbulence and its evolution with the Weissenberg number depart significantly from the Newtonian case. For these reasons only a qualitative (and most probably incomplete) comparison with the case of a Newtonian swirling flow will be attempted below. Corresponding to $\operatorname{Pr}=1$ and $R e=2000$ the numerical simulations presented by Iwatsu in Ref. Iwatsu [2004] for the von Karman swirling flow of a Newtonian fluid predict a maximal increase of the heat transport efficiency (quantified by the space averaged Nusselt number) of roughly 9 times corresponding to $R i=0.01$. As they increase the Richardson number, however, the increase in the transport efficiency at $R e=2000$ decreases down to roughly 1.5 times corresponding to $R i=1$, see Fig. 15 in Ref. Iwatsu [2004]. Similar numerical results are found by Kim and Hyun in Refs. Kim and Hyun [1997b,c] for $\operatorname{Pr}=0.7, R e=2000$ and $R i$ ranging from 0.01 to 10 .

The central conclusion of this part is that the efficiency of the heat transport by elastic turbulence is comparable in magnitude to that of the Newtonian counterpart studied by others although during our experiments both $R i$ and $\operatorname{Pr}$ were larger. In addition to that, based on the data presented in Fig. 74 the intensity of the heat transport by elastic turbulence is practically insensitive to these non-dimensional numbers if $W i$ is sufficiently large. This is a significant difference (and perhaps an advantage) with respect to the swirling flow of a Newtonian fluid where an increase of both $\operatorname{Pr}$ and $R i$ translates into a decrease of the transport efficiency. As the elastic turbulent flow field has a single relevant spatial scale set by the dimensions of the fluid container FC and there exists no physical equivalent of the cascade towards smaller scales observed in the inertial turbulence, a natural question to be answered in the following is how does the intensity 


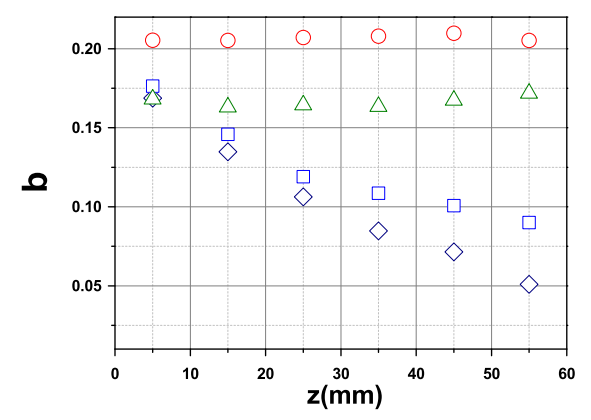

Figure 75. Dependence of the intensity factor $b$ on the vertical coordinate. The symbols refer to the Weissenberg number: rhombs $(\diamond)-W i=0$, squares $(\square)-W i=0.8$, triangles $(\triangle)-W i=7.7$, circle $(\circ)-W i=15.4$.

of the heat transfer depend on the position $z$ in the flow. To address this point, we plot in Fig. 75 the local intensity factor $b$ versus the vertical position $z$ for various Weissenberg numbers spanning the relevant flow regimes. In the absence of a flow $(W i=0)$ when the heat is transported solely by thermal conduction, a strong spatial dependence of the intensity factor is observed (the rhombs in Fig. 75). As expected for a purely conductive case, the intensity of the heat transport decreases monotonically as one moves away from the heat sink located at $z=0 \mathrm{~cm}$. A slight increase of the local intensity factor is observed within the laminar and steady flow regime $(W i=0.8)$, particularly in the proximity of the top driving disk $\mathbf{D}$ (the squares). This slight increase in the intensity of the heat transfer process may be explained by the presence of the steady Ekman vortex induced by the array of the thermocouples (Fig. 72). A notable increase of the heat transport intensity is observed at any measuring position within the transitional and the fully developed elastic turbulent regimes (the triangles and the circles). We point out that within these flow regimes the intensity is independent on the flow coordinate consistently with a full homogenisation of the temperature field. The largest increase in the local intensity is observed near the top disk $\mathbf{D}$ and accounts for nearly 4 times the intensity measured in a purely conductive state, Fig. 75 .

\subsection{Statistical and scaling properties of the temperature fluctu- ations: passive or active scalar?}

In a random flow the scalar temperature field may act either as a passive scalar when it does not react back on the flow field or, if it does, as an active 
scalar. A classical example where the temperature acts as an active scalar is that of a fluid heated from below: the density gradient may induce a thermoconvective instability and thus the temperature reacts back on the flow field. In the passive case the homogenisation of the temperature field by a random and spatially smooth flow field in a regime of Elastic Turbulence is similar to the mixing problem studied in detail in Refs. Burghelea et al. [2004a,c], Burghelea [2005] and several fundamental features have been highlighted:

1. The probability distributions of the passive scalar fluctuations have exponential tails and the second order moment of these distributions decays exponentially in time.

2. The temporal correlations of the passive scalar fluctuations decay over times of the same order of magnitude with the correlation times of the flow field and the largest relaxation time of the polymer molecules.

3. The spectra of the passive scalar fluctuations decay algebraically, $P \propto$ $k^{\delta}$, with $\delta \approx-1$

For all the experiments we reported above, the fluid has been cooled from below. Thus, thermo-convective instabilities are ruled out and, at least at a first glance, one would be tempted to believe that the temperature field acts as a passive scalar meaning that the heat transport process is physically equivalent to the mixing problem. At a more careful inspection this is actually not granted. As illustrated in Fig. 70, the rheological properties (shear viscosity and relaxation time) are strongly dependent on the temperature. Thus, the temperature field is directly coupled to the stress tensor which may in turn act on the flow field. Thus, whether the temperature acts as a passive or an active scalar remains to be investigated experimentally which sets the main scope of this section.

To get a first insight into the statistics of the temperature fluctuations at various Weissenberg numbers, we extract the fluctuating part of each time series of the reduced temperature presented in Fig. 73(b) by subtracting from the original signal its pedestal $\mathcal{P}$ obtained via a fifth degree polynomial fit, $\theta_{r}\left(t / t_{d}\right)=\theta\left(t / t_{d}\right)-\mathcal{P}\left(t / t_{d}\right)$.

As by in situ measurements of the apparent viscosity $\eta_{a}$ of the polymer solution during the heat transfer process we have observed that at high Weissenberg numbers the mechanical degradation occurs (manifested through a significant decrease of $\left.\eta_{a}\right)$ at very late stages of the process $\left(t / t_{d}>0.5\right)$, the whole statistical analysis we present below was restricted to time windows within the logarithmic scaling range, $0.1<t / t_{d}<0.3$ and no conclusions were drawn from the temperature data acquired beyond this range. In the absence of a flow $(W i=0)$ the pedestal of the signal characterises the purely conductive heat transport and in a laminar regime $\left(0<W i<W i_{c}\right)$ 


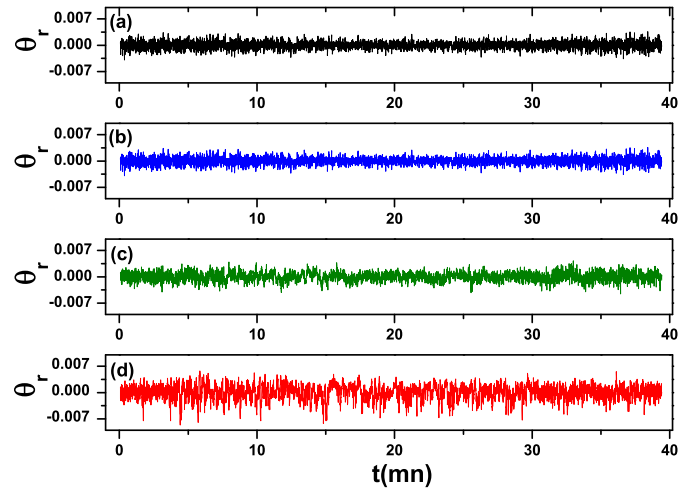

Figure 76. Time series of the fluctuating part of the reduced temperature $\theta_{r}$ obtained from the measurements of the thermocouple $T_{4}$ (see Fig. 69 ) at various Weissenberg numbers: (a) $W i=0$ (b) $W i=0.8$, (c) $W i=7.7$, (d) $W i=15.4$

it describes the summed contributions of the conduction and the laminar convective transport carried on by the stable vortex illustrated in Fig. 72 . Several time series of the fluctuating part of the reduced temperature $\theta_{r}$ obtained according to the procedure described above from the raw data acquired by the thermocouple $T_{4}$ positioned at $r=R_{c} / 2$ (see Fig. 69) at various Weissenberg numbers increasing from the top to the bottom are presented in Fig. 76.

In the absence of a flow $(W i=0)$ and below the onset of the primary elastic instability $(W i=0.8)$ these fluctuations are of a purely instrumental nature. Upon an increase of the Weissenberg number within the fully developed elastic turbulent regime a significant increase of the level of fluctuations beyond the instrumental level is observed, Fig. $76(\mathrm{c}, \mathrm{d})$. At $W i=15.4$ the randomly fluctuating temperature time series also exhibits some intermittency.

The spatial distribution of the root mean squared of the fluctuations of the reduced temperature within each flow regime is illustrated in Fig. 77.

In the absence of a flow and within the laminar regime, no dependence of the level of the fluctuations on the vertical coordinate $z$ can be observed (which was expected, as the six thermocouples are identical, i.e. the instrumental error of their measurements is the same). Within the transitional regime and the fully developed elastic turbulent regime a clear spatial dependence of the temperature fluctuations can be observed (the triangles and 


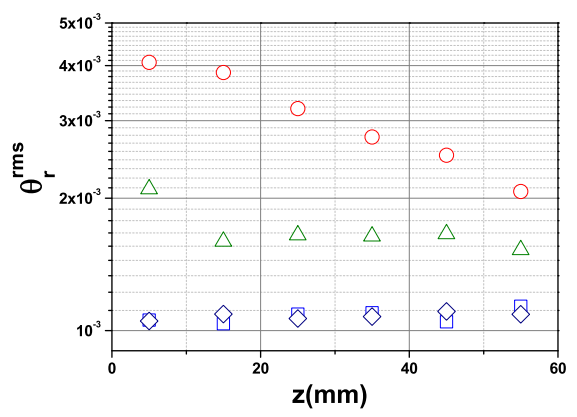

Figure 77. Space distribution of the rms of the fluctuations of the reduced temperature, $\theta_{r}^{r m s}$ measured at various Weissenberg numbers: rhombs $(\diamond)$ - $W i=0$, squares $(\square)$ - $W i=0.8$, triangles $(\triangle)$ - $W i=7.7$, circle $(\circ)$ $W i=15.4$.

the squares in Fig. 77). Due to the particular topology of the randomly fluctuating spiral vortex illustrated in Fig. 72 at $W i=15.4$, the fluctuations are the largest in the vicinity of the bottom plate of the flow container FC and decrease monotonically as one approaches the level of the top driving disk D. Near the bottom plate, the temperature fluctuations due to the advection of the temperature field by the random flow field are about 3.5 times larger than the instrumental fluctuations observed in the laminar regime. We point out that in spite of the clear anisotropy of the temperature fluctuations within the fully developed elastic turbulent regime, the intensity of the heat transport quantified by the parameter $b$ introduced above is practically independent on the spatial coordinate (the circles in Fig. 75).

The dependence of the level of fluctuations measured in the fully developed elastic turbulent regime near the bottom plate of the fluid container on the vertical coordinate indicates the possible existence of a boundary layer for the heat transfer with its edge roughly located around $z=15 \mathrm{~mm}$, Fig. 75. As the spatial resolution of our measuring technique along the vertical direction is limited and the construction of the experimental apparatus does not allow one to move the thermocouples along this direction, a systematic investigation of this fact similar to that performed for the velocity boundary layer presented in Ref. Burghelea et al. [2007] or the passive scalar boundary layer presented in Ref. Burghelea et al. [2004a] was not possible and should to be addressed by future experimental studies.

The probability density functions (pdf's) of the fluctuations of the reduced temperature measured by the thermocouple $T_{4}$ are presented in Fig. 78(a). In the absence of a flow (the rhombs) and within a laminar flow 


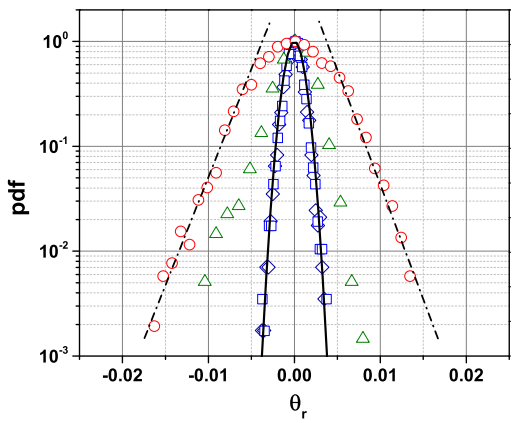

(a)

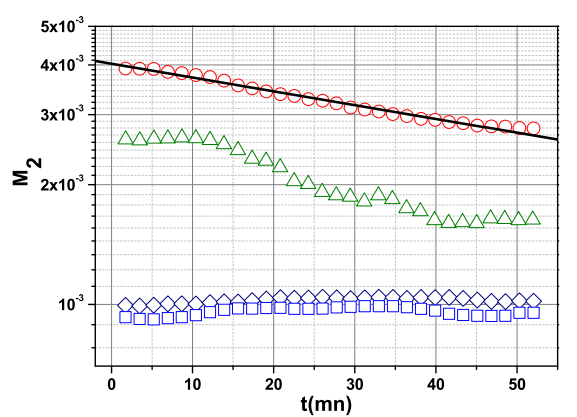

(b)

Figure 78. (a) Probability density functions (pdf's) of the fluctuations of the reduced temperature, $T_{r}^{r m s}$, obtained from the measurements of the thermocouple $T_{4}$. The full line is a Gaussian fit, the dash-dotted lines are guides for eye highlighting the exponential tails of the pdf's. (b) Time dependence of the second order moment $M_{2}$ of the distribution of the temperature fluctuations obtained from the measurements of the thermocouple $T_{4}$. The full line is an exponential decay fit, $M_{2} \propto e^{-t / 7523}$. In both panels, the symbols refer to different $W i$ : rhombs $(\diamond)-W i=0$, squares $(\square)$ $W i=0.8$, triangles $(\triangle)-W i=7.7$, circles $(\circ)-W i=15.4$.

regime (the squares) the pdf's can be fitted by a Gaussian function (the full line).

Upon an increase of the Weissenberg number beyond the onset of the primary elastic instability (the triangles and the circles) the distributions become significantly broader and exhibit exponential tails (see the dash-dot lines).

A certain degree of asymmetry of the pdf's which may be associated to the intermittency of the temperature fluctuations visible in Fig. 76(c-d) can be equally observed.

The decay of the second order moments $M_{2}$ of the probability density functions is illustrated in Fig. 78(b). Within the elastic turbulent regime (the circles) an exponential decay of the second order moment is observed, $M_{2} \propto e^{-t / t_{m i x}}$. Here $t_{m i x}$ is a characteristic time scale for the decay of the temperature fluctuations and is equivalent to the mixing time in the passive scalar problem. By an exponential fit of the data presented in Fig. 78 (b) one obtains $t_{m i x} \approx 7523 \mathrm{~s}$ which is about 3.4 times smaller than the characteristic time diffusion time $t_{d}$. This result corroborates with the 3.5 times increase in the efficiency of the heat transfer quantified by the slope 

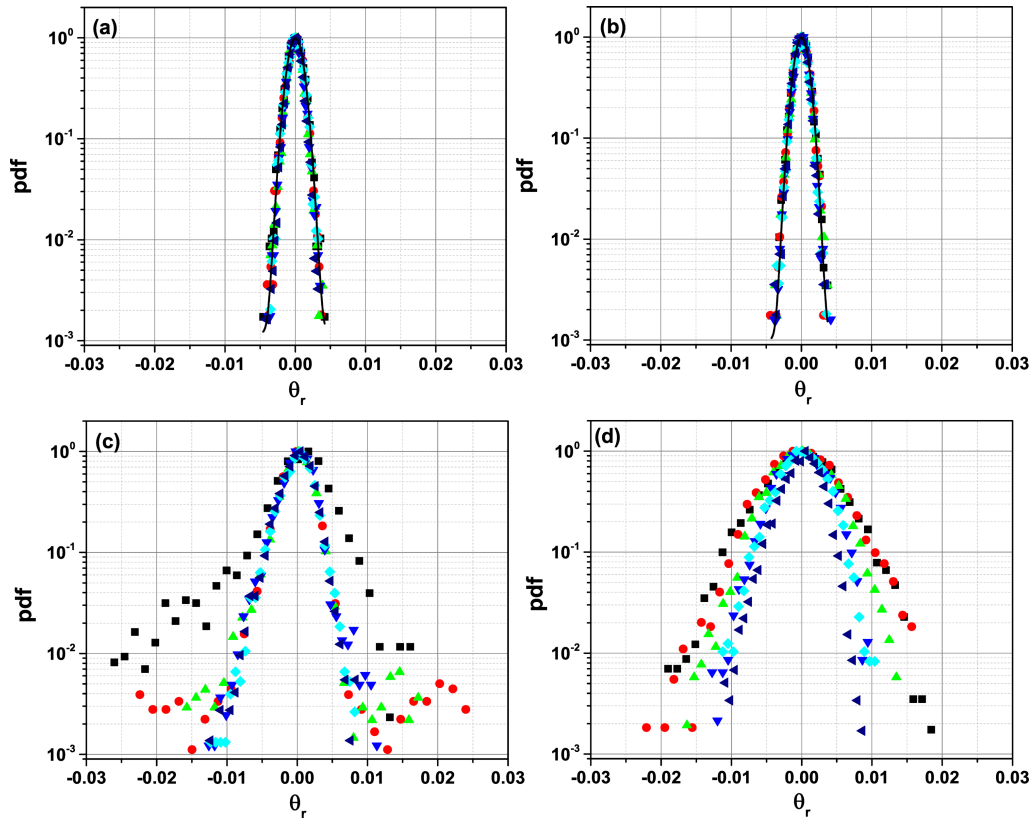

Figure 79. Pdf's of the fluctuations of the reduced temperature measured at various vertical positions and several $W i$ : (a) $W i=0$ (b) $W i=0.8$ (c) $W i=7.7$ (d) $W i=15.4$. In each panel the symbols refer to different thermocouples (see Fig. 69): squares $(\mathbf{\square})-T_{1}$, circles $(\bullet)-T_{2}$, up triangles $(\boldsymbol{\Delta})-T_{3}$, down triangles $(\mathbf{\nabla})-T_{4}$, rhombs $(\diamond)-T_{5}$, left triangles $(\triangleleft)-T_{6}$. The full lines in panels $(a, b)$ are Gaussian fitting functions.

$b$ and illustrated in Figs. 74, 75 .

The pdf's of the fluctuations of the reduced temperature measured at each vertical position for various $W i$ are presented in Fig. 79. In the absence of a flow and within the laminar regime the pdf's acquired at each of the six vertical positions collapse onto a single master curve which can be well fitted by a Gaussian distribution (panels (a-b)).

Within the transitional regime $(W i=7.7)$ a strongly intermittent distribution is observed particularly in the vicinity of the bottom plate (the squares and the circles in panel (c)). The degree of intermittency decreases as one approaches the top disk but does not vanish.

In the fully developed elastic turbulent regime the pdf's exhibit an intermittent behaviour and exponential tails regardless the vertical position, 


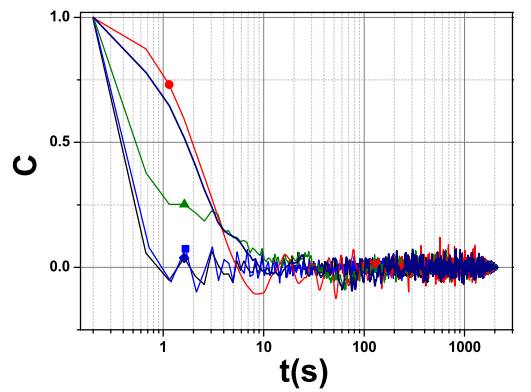

(a)

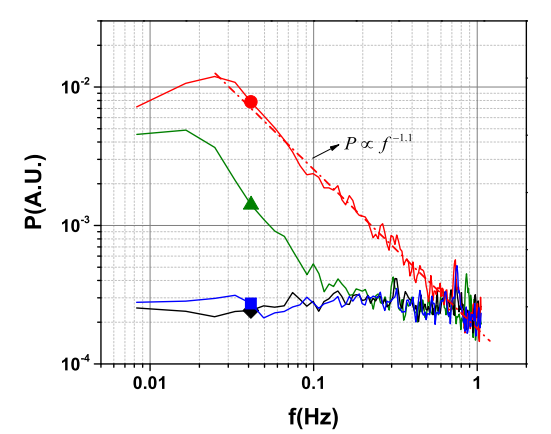

(b)

Figure 80. 80(a) Temporal autocorrelation functions $C$ of the fluctuations of the reduced temperature .(b) Spectra of the fluctuations of the reduced temperature. The data were measured by the thermocouple $T_{4}$ (see Fig. 69). The symbols in both panels refer to various Weissenberg numbers: rhombs $(\bullet)-W i=0$, squares $(\boldsymbol{\square})-W i=0.8$, triangles $(\boldsymbol{\Delta})-W i=7.7$, circle $(\bullet)-W i=15.4$. The dash - dot line is a guide for the eye, $P \propto f^{-1.1}$.

panel (d).

These results on the statistics of the fluctuations of the reduced temperature presented above are in a good agreement with the theoretical prediction for the decay of a passive scalar in a random smooth flow, Chertkov et al. [1995a], Balkovsky and Fouxon [1999], and with the experiments on the mixing of a passive scalar in a macroscopic curvilinear channel, Groisman and Steinberg [2001], and in a micro channel, Burghelea et al. [2004c,a].

The temporal auto-correlation functions of the fluctuations of the reduced temperature measured within each relevant flow regime are presented in Fig. 80(a).

In the absence of a flow and within the laminar regime the fluctuations of the reduced temperature are solely related to the instrumental noise of the temperature measurements and, consequently, the signals are short correlated (the curves labeled by a rhomb and a square in Fig. 80(a)). Within the transitional and the fully developed elastic turbulent regimes the reduced temperature de-correlates over characteristic times $\tau_{c}$ comparable to the relaxation time of the polymer $\lambda$. This behaviour is quite similar to the behaviour of the time autocorrelation functions of the azimuthal velocity component, see Fig. 24 in Ref. Burghelea et al. [2007] which is probably due to the coupling between the flow field and the temperature field. 


\section{Bibliography}

Narasimha Acharya, Mihir Sen, and Chang Hsueh-Chia. Heat transfer enhancement in coiled tubes by chaotic mixing. International Journal of Heat and Mass Transfer, 35(10):2475 - 2489, 1992. ISSN 0017-9310. doi: http://dx.doi.org/10.1016/0017-9310(92)90090-F. URL http://www.sciencedirect.com/science/article/pii/001793109290090F.

Y. Amarouchene and H. Kellay. Batchelor scaling in fastflowing soap films. Phys. Rev. Lett., 93:214504, Nov 2004. doi: 10.1103/PhysRevLett.93.214504. URL https://link.aps.org/doi/10.1103/PhysRevLett.93.214504.

Hassan Aref. Chaotic advection of fluid particles. Philosophical Transactions of the Royal Society of London. Series A: Physical and Engineering Sciences, 333(1631):273-288, 1990. doi: 10.1098/rsta.1990.0161. URL http://rsta.royalsocietypublishing. org/content/333/1631/273. abstract.

V. Artale, G. Boffetta, A. Celani, M. Cencini, and A. Vulpiani. Dispersion of passive tracers in closed basins: Beyond the diffusion coefficient. Physics of Fluids, 9(11):3162-3171, 1997. doi: 10.1063/1.869433. URL https://doi.org/10.1063/1.869433.

E. Balkovsky and A. Fouxon. Universal long-time properties of lagrangian statistics in the batchelor regime and their application to the passive scalar problem. Phys. Rev. E, 60: 4164-4174, Oct 1999. doi: 10.1103/PhysRevE.60.4164. URL http://link.aps.org/doi/10.1103/PhysRevE.60.4164.

E. Balkovsky, A. Fouxon, and V. Lebedev. Turbulence of polymer solutions. Phys. Rev. E, 64:056301, Oct 2001. doi: 10.1103/PhysRevE.64.056301. URL http://link.aps.org/doi/10.1103/PhysRevE.64.056301.

G. K. Batchelor. Small-scale variation of convected quantities like temperature in turbulent fluid part 1. general discussion and the case of small conductivity. Journal of Fluid Mechanics, 5(1):113-133, 1959a. doi: 10.1017/S002211205900009X.

G. K. Batchelor. Small-scale variation of convected quantities like temperature in turbulent fluid part 1. general discussion and the case of small conductivity. Journal of Fluid Mechanics, 5(1): 113?133, Jan 1959b. doi: 10.1017/S002211205900009X. URL https://www . cambridge.org/core/article/small-scale-variation-of-convected-quantities-like-te

Andrew Belmonte, Brian Martin, and Walter I. Goldburg. Experimental study of taylor's hypothesis in a turbulent soap film. Physics of Fluids, 12(4):835-845, 2000. doi: 10.1063/1.870339. URL https://doi.org/10.1063/1.870339.

R.B. Bird, O. Hassager, R.C. Armstrong, and C.F. Curtiss. Dynamics of Polymeric Liquids, vol. 1. Wiley, New York, 1977. 
G. Boffetta, M. Cencini, M. Falcioni, and A. Vulpiani. Predictability: a way to characterize complexity. Physics Reports, 356(6):367 - 474, 2002. ISSN 0370-1573. doi: https://doi.org/10.1016/S0370-1573(01)00025-4. URL http://www.sciencedirect.com/science/article/pii/S0370157301000254.

Guido Boffetta, Antonio Celani, and Stefano Musacchio. Two-dimensional turbulence of dilute polymer solutions. Phys. Rev. Lett., 91: 034501, Jul 2003. doi: 10.1103/PhysRevLett.91.034501. URL https://link.aps.org/doi/10.1103/PhysRevLett.91.034501.

Boffetta, G., Cencini, M., Espa, S., and Querzoli, G. Experimental evidence of chaotic advection in a convective flow. Europhys. Lett., 48(6):629-633, 1999. doi: 10.1209/epl/i1999-00530-3. URL https://doi.org/10.1209/epl/i1999-00530-3.

A. Brandstäter, J. Swift, Harry L. Swinney, A. Wolf, J. Doyne Farmer, Erica Jen, and P. J. Crutchfield. Low-dimensional chaos in a hydrodynamic system. Phys. Rev. Lett., 51:14421445, Oct 1983. doi: 10.1103/PhysRevLett.51.1442. URL https://link.aps.org/doi/10.1103/PhysRevLett.51.1442.

T Burghelea, E Segre, and V Steinberg. Mixing by polymers: Experimental test of decay regime of mixing. Phys. Rev. Lett., 92(16):164501-4, April 2004a.

T. Burghelea, E. Segre, and V. Steinberg. Statistics of particle pair separations in the elastic turbulent flow of a dilute polymer solution. EPL (Europhysics Letters), 68(4):529, 2004b. URL http://stacks. iop.org/0295-5075/68/i=4/a=529.

T. Burghelea, E. Segre, and V. Steinberg. Validity of the Taylor hypothesis in a random spatially smooth flow. Physics of Fluids, 17(10):1031011, 103101-8, 2005. doi: http://dx.doi.org/10.1063/1.2077367. URL http://scitation.aip.org/content/aip/journal/pof2/17/10/10.1063/1.2077367.

Teodor Burghelea. Elastic turbulence and mixing in a dilute polymer solution. PhD thesis, Feinberg Graduate School of the Weizmann Institute of Science, 2005.

Teodor Burghelea, Enrico Segre, Israel Bar-Joseph, Alex Groisman, and Victor Steinberg. Chaotic flow and efficient mixing in a microchannel with a polymer solution. Phys. Rev. E, 69(6):066305-8, June 2004c.

Teodor Burghelea, Enrico Segre, and Victor Steinberg. Elastic turbulence in von Karman swirling flow between two disks. Physics of Fluids, 19(5):053104, 2007. doi: http://dx.doi.org/10.1063/1.2732234. URL http://scitation.aip.org/content/aip/journal/pof2/19/5/10.1063/1.2732234.

Burghelea, T, Segre, E, and Steinberg, V. Validity of the Taylor hypothesis in a random spatially smooth flow. Phys. Fluids, 17(10):103101-8, 2005. 
Burghelea, Teodor, Segre, Enrico, and Steinberg, Victor. Role of Elastic Stress in Statistical and Scaling Properties of Elastic Turbulence. Phys. ^Rev. ${ }^{2}$ Lett., 96(21):214502-4, May 2006.

M. Chertkov. On how a joint interaction of two innocent partners (smooth advection and linear damping) produces a strong intermittency. Physics of Fluids, 10(11):30173019, 1998. doi: http://dx.doi.org/10.1063/1.869826. URL http://scitation.aip.org/content/aip/journal/pof2/10/11/10.1063/1.869826.

M. Chertkov and V. Lebedev. Decay of scalar turbulence revisited. Phys. Rev. Lett., 90:034501, Jan 2003. doi: 10.1103/PhysRevLett.90.034501. URL https://link.aps.org/doi/10.1103/PhysRevLett.90.034501.

M. Chertkov, G. Falkovich, I. Kolokolov, and V. Lebedev. Statistics of a passive scalar advected by a large-scale two-dimensional velocity field: Analytic solution. Phys. Rev. E, 51:56095627, Jun 1995a. doi: 10.1103/PhysRevE.51.5609. URL http://link.aps.org/doi/10.1103/PhysRevE.51.5609.

M. Chertkov, G. Falkovich, I. Kolokolov, and V. Lebedev. Statistics of a passive scalar advected by a large-scale two-dimensional velocity field: Analytic solution. Phys. Rev. E, 51:56095627, Jun 1995b. doi: 10.1103/PhysRevE.51.5609. URL https://link.aps.org/doi/10.1103/PhysRevE.51.5609.

Hou-Pu Chou, Charles Spence, Axel Scherer, and Stephen Quake. A microfabricated device for sizing and sorting dna molecules. Proceedings of the National Academy of Sciences, 96(1):11-13, 1999. ISSN 0027-8424. doi: 10.1073/pnas.96.1.11. URL https ://www . pnas . org/content/96/1/11.

Werner J. A. Dahm and Kenneth B. Southerland. Experimental assessment of taylor's hypothesis and its applicability to dissipation estimates in turbulent flows. Physics of Fluids, 9(7):2101-2107, 1997. doi: 10.1063/1.869329. URL https://doi.org/10.1063/1.869329.

P. G. De Gennes. Coil-stretch transition of dilute flexible polymers under ultrahigh velocity gradients. The Journal of Chemical Physics, 60(12):5030-5042, 1974. doi: 10.1063/1.1681018. URL https://doi.org/10.1063/1.1681018.

V. W. Ekman. On the influence of the earth's rotation on ocean currents. Ark. Mat, Astron. Fys., 2(11):1 - 53, 1905.

Kamal El Omari and Yves Le Guer. Thermal chaotic mixing of powerlaw fluids in a mixer with alternately rotating walls. Journal of Non-Newtonian Fluid Mechanics, 165(11-12):641 - 651, 2010. ISSN 0377-0257. doi: http://dx.doi.org/10.1016/j.jnnfm.2010.03.004. URL http://www.sciencedirect.com/science/article/pii/S037702571000090X. 
G. Falkovich, K. Gawędzki, and M. Vergassola. Particles and fields in fluid turbulence. Rev. Mod. Phys., 73:913975, Nov 2001. doi: 10.1103/RevModPhys.73.913. URL https://link.aps.org/doi/10.1103/RevModPhys.73.913.

M. J. Fisher and P. O. A. L. Davies. Correlation measurements in a nonfrozen pattern of turbulence. Journal of Fluid Mechanics, 18(1):97-116, 1964. doi: $10.1017 /$ S0022112064000076.

A. Fouxon and V. Lebedev. Spectra of turbulence in dilute polymer solutions. Physics of Fluids, 15(7):2060-2072, 2003. doi: http://dx.doi.org/10.1063/1.1577563. URL http://scitation.aip.org/content/aip/journal/pof2/15/7/10.1063/1.1577563.

Gerashchenko, S., Chevallard, C., and Steinberg, V. Single-polymer dynamics: Coil-stretch transition in a random flow. Europhys. Lett., 71(2):221-227, 2005. doi: 10.1209/epl/i2005-10087-1. URL https://doi.org/10.1209/epl/i2005-10087-1.

J. P. Gollub, J. Clarke, M. Gharib, B. Lane, and O. N. Mesquita. Fluctuations and transport in a stirred fluid with a mean gradient. Phys. Rev. Lett., 67:3507-3510, Dec 1991a. doi: 10.1103/PhysRevLett.67.3507. URL https://link.aps.org/doi/10.1103/PhysRevLett.67.3507.

J. P. Gollub, J. Clarke, M. Gharib, B. Lane, and O. N. Mesquita. Fluctuations and transport in a stirred fluid with a mean gradient. Phys. Rev. Lett., 67:3507-3510, Dec 1991b. doi: 10.1103/PhysRevLett.67.3507. URL http://link.aps.org/doi/10.1103/PhysRevLett.67.3507.

Alexander Groisman and Victor Steinberg. Mechanism of elastic instability in couette flow of polymer solutions: Experiment. Physics of Fluids, 10 (10):2451-2463, 1998. doi: http://dx.doi.org/10.1063/1.869764. URL http://scitation.aip.org/content/aip/journal/pof2/10/10/10.1063/1.869764.

Alexander Groisman and Victor Steinberg. Elastic turbulence in a polymer solution flow. Nature, 405(6782):53-55, 2000.

Alexander Groisman and Victor Steinberg. Efficient mixing at low reynolds numbers using polymer additives. Nature, 410(6831):905-908, 2001.

Alexander Groisman and Victor Steinberg. Elastic turbulence in curvilinear flows of polymer solutions. New Journal of Physics, 6(1):29, 2004. URL http://stacks.iop.org/1367-2630/6/i=1/a=029.

Silas E. Gustafsson. Transient plane source techniques for thermal conductivity and thermal diffusivity measurements of solid materials. Review of Scientific Instruments, 62(3):797-804, 1991. doi: 10.1063/1.1142087. URL http://dx.doi.org/10.1063/1.1142087.

Mattias Gustavsson, Ernest Karawacki, and Silas E. Gustafsson. Thermal conductivity, thermal diffusivity, and specific heat of thin samples from transient measurements with hot disk sensors. Review of Scientific Instruments, 65(12):3856-3859, 1994. doi: 10.1063/1.1145178. URL http://dx.doi.org/10.1063/1.1145178. 
Carl L. Hansen, Emmanuel Skordalakes, James M. Berger, and Stephen R. Quake. A robust and scalable microfluidic metering method that allows protein crystal growth by free interface diffusion. Proceedings of the National Academy of Sciences, 99(26):16531-16536, 2002. ISSN 0027-8424. doi: 10.1073/pnas.262485199. URL https://www . pnas.org/content/99/26/16531.

H.C. Hershey and Z. L. Zalkin. Existence of two types of drag reduction in pipe flow of dilute polymer solutions. Number 3. 1967.

D.M Hobbs and F.J Muzzio. The kenics static mixer: a three-dimensional chaotic flow. Chemical Engineering Journal, 67(3):153 - 166, 1997. ISSN 1385-8947. doi: http://dx.doi.org/10.1016/S1385-8947(97)00013-2. URL http://www. sciencedirect. com/science/article/pii/S1385894797000132.

Reima Iwatsu. Flow pattern and heat transfer of swirling flows in cylindrical container with rotating top and stable temperature gradient. International Journal of Heat and Mass Transfer, 47(12-13):2755 - 2767, 2004.

Marie-Caroline Jullien, Patrizia Castiglione, and Patrick Tabeling. Experimental observation of batchelor dispersion of passive tracers. Phys. Rev. Lett., 85:3636-3639, Oct 2000. doi: 10.1103/PhysRevLett.85.3636. URL https://link.aps.org/doi/10.1103/PhysRevLett.85.3636.

Yonggun Jun and Victor Steinberg. Mixing of passive tracers in the decay batchelor regime of a channel flow. Physics of Fluids, 22 (12):123101, 2010. doi: http://dx.doi.org/10.1063/1.3522400. URL http://scitation. aip.org/content/aip/journal/pof2/22/12/10.1063/1.3522400; jsessionid=vicLijK

Yonggun Jun and Victor Steinberg. Elastic turbulence in a curvilinear channel flow. Phys. Rev. E, 84:056325, Nov 2011. doi: 10.1103/PhysRevE.84.056325. URL http://link.aps.org/doi/10.1103/PhysRevE.84.056325.

Won Nyun Kim and Jae Min Hyun. Convective heat transfer in a cylinder with a rotating lid under stable stratification. International Journal of Heat and Fluid Flow, 18(4):384 - 388, 1997a. ISSN 0142727X. doi: http://dx.doi.org/10.1016/S0142-727X(97)00012-X. URL http://www. sciencedirect.com/science/article/pii/S0142727X9700012X.

Won Nyun Kim and Jae Min Hyun. Convective heat transfer in a cylinder with a rotating lid under stable stratification. International Journal of Heat and Fluid Flow, 18(4):384-388, 1997b. doi: http://dx.doi.org/10.1016/S0142-727X(97)00012-X. URL http://www. sciencedirect.com/science/article/pii/S0142727X9700012X.

Won Nyun Kim and Jae Min Hyun. Convective heat transfer in a cylinder with a rotating lid under stable stratification. International Journal of Heat and Fluid Flow, 18(4):384 - 388, 1997c. ISSN 0142727X. doi: http://dx.doi.org/10.1016/S0142-727X(97)00012-X. URL http://www.sciencedirect.com/science/article/pii/S0142727X9700012X. 
Konrad Koeltzsch. On the relationship between the lagrangian and eulerian time scale. Atmospheric Environment, 33(1):117 - 128, 1998. ISSN 1352-2310. doi: https://doi.org/10.1016/S1352-2310(98)00135-6. URL http://www.sciencedirect.com/science/article/pii/S1352231098001356.

Robert H. Kraichnan. Small-scale structure of a scalar field convected by turbulence. The Physics of Flu$i d s, \quad 11(5): 945-953, \quad 1968 . \quad$ doi: 10.1063/1.1692063. URL https://aip.scitation.org/doi/abs/10.1063/1.1692063.

P.-A. Krogstad, J. H. Kaspersen, and S. Rimestad. Convection velocities in a turbulent boundary layer. Physics of Fluids, 10(4):949-957, 1998. doi: 10.1063/1.869617. URL https://doi.org/10.1063/1.869617.

W. M. Kulicke, M Koter, and H. and Grager. Polymer Characterization /Polymer Solutions. Springer Verlag, Berlin, 1989.

G. Lacorata, E. Aurell, and A. Vulpiani. Drifter dispersion in the adriatic sea: Lagrangian data and chaotic model. Annales Geophysicae, 19(1):121-129, 2001. doi: 10.5194/angeo-19-121-2001. URL https://www . ann-geophys . net/19/121/2001/.

L. D. Landau and E. M. Lisfshits. Statistical Physics, Part 1: Volume 5 (Course of Theoretical Physics, Volume 5). Butterworth-Heinemann, third edition edition, 1980. ISBN 0750633727.

B. R. Lane, O. N. Mesquita, S. R. Meyers, and J. P. Gollub. Probability distributions and thermal transport in a turbulent grid flow. Physics of Fluids A: Fluid Dynamics, 5(9):2255-2263, 1993. doi: 10.1063/1.858564. URL http://dx.doi.org/10.1063/1.858564.

R. G. Larson. Instabilities in viscoelastic flows. Rheologica Acta, 31(3): 213-263, 1992. ISSN 1435-1528. doi: 10.1007/BF00366504. URL http://dx.doi.org/10.1007/BF00366504.

Chang Ho Lee and Jae Min Hyun. Flow of a stratified fluid in a cylinder with a rotating lid. International Journal of Heat and Fluid Flow, 20(1):26 - 33, 1999. URL http://www.sciencedirect.com/science/article/pii/S0142727X98100413.

M. Lesieur. Turbulence in fluids. Springer, 2008.

Dong-Yang Li, Xiao-Bin Li, Hong-Na Zhang, Feng-Chen Li, Shizhi Qian, and Sang Woo Joo. Efficient heat transfer enhancement by elastic turbulence with polymer solution in a curved microchannel. Microfluidics and Nanofluidics, 21(1):10, 2017. ISSN 1613 - 4990.

Noo Li Jeon, Harihara Baskaran, Stephan K. W. Dertinger, George M. Whitesides, Livingston Van De Water, and Mehmet Toner. Neutrophil chemotaxis in linear and complex gradients of interleukin- 8 formed in a microfabricated device. Nature Biotechnology, 20:826 EP -, 072002. URL https : //doi .org/10.1038/nbt712. 
C. C. Lin. On taylor's hypothesis and the acceleration terms in the navierstokes equation. QUARTERLY OF APPLIED MATHEMATICS, pages 295-306, 1953.

Yonggang Liu, Yonggun Jun, and Victor Steinberg. Longest relaxation times of double-stranded and single-stranded dna. Macromolecules, 40(6):2172-2176, 2007. doi: 10.1021/ma062715d. URL http://pubs.acs.org/doi/abs/10.1021/ma062715d.

Yonggang Liu, Yonggun Jun, and Victor Steinberg. Concentration dependence of the longest relaxation times of dilute and semidilute polymer solutions. Journal of Rheology (1978-present), 53 (5):1069-1085, 2009. doi: http://dx.doi.org/10.1122/1.3160734. URL http://scitation. aip.org/content/sor/journal/jor2/53/5/10.1122/1.3160734.

J. L. Lumley. Interpretation of time spectra measured in high-intensity shear flows. The Physics of Fluids, 8 (6):1056-1062, 1965 . doi: 10.1063/1.1761355. URL https://aip.scitation.org/doi/abs/10.1063/1.1761355.

J.J. Magda and R.G. Larson. A transition occurring in ideal elastic liquids during shear flow. Journal of Non-Newtonian Fluid Mechanics, 30(1):1 - 19, $1988 . \quad$ ISSN 0377-0257. doi: https://doi.org/10.1016/0377-0257(88)80014-4. URL http://www.sciencedirect.com/science/article/pii/0377025788800144.

Hanbin Mao, Paul S. Cremer, and Michael D. Manson. A sensitive, versatile microfluidic assay for bacterial chemotaxis. Proceedings of the National Academy of Sciences, 100(9):5449-5454, 2003. ISSN 0027-8424. doi: 10.1073/pnas.0931258100. URL https ://www . pnas . org/content/100/9/5449.

Gareth H McKinley, Peyman Pakdel, and Alparslan Öztekin. Rheological and geometric scaling of purely elastic flow instabilities. Journal of NonNewtonian Fluid Mechanics, 67:19-47, November 1996.

J. Mi and R. A. Antonia. Corrections to taylor's hypothesis in a turbulent circular jet. Physics of Fluids, 6(4):1548-1552, 1994 . doi: 10.1063/1.868268. URL https://doi.org/10.1063/1.868268.

Alexander N. Morozov and Wim van Saarloos. An introductory essay on subcritical instabilities and the transition to turbulence in visco-elastic parallel shear flows. Physics Reports, 447(3-6):112 - 143, 2007. ISSN 0370-1573. doi: http://dx.doi.org/10.1016/j.physrep.2007.03.004. URL http://www.sciencedirect.com/science/article/pii/S0370157307001469. Nonequilibrium physics: From complex fluids to biological systems I. Instabilities and pattern formation.

J. P. B. Mota, A. J. S. Rodrigo, and E. Saatdjian. Optimization of heat-transfer rate into time-periodic two-dimensional stokes 
flows. International Journal for Numerical Methods in Fluids, 53 (6):915-931, 2007. ISSN 1097-0363. doi: 10.1002/fld.1312. URL http://dx.doi.org/10.1002/fld.1312.

S. J. Muller, R. G. Larson, and E. S. G. Shaqfeh. A purely elastic transition in taylor-couette flow. Rheologica Acta, 28(6):499503, 1989a. ISSN 1435-1528. doi: 10.1007/BF01332920. URL http://dx.doi.org/10.1007/BF01332920.

S. J. Muller, R. G. Larson, and E. S. G. Shaqfeh. A purely elastic transition in taylor-couette flow. Rheologica Acta, 28(6):499-503, 1989b. doi: 10.1007/BF01332920. URL https://doi.org/10.1007/BF01332920.

M. H. Oddy, J. G. Santiago, and J. C. Mikkelsen. Electrokinetic instability micromixing. Analytical Chemistry, 73(24):5822-5832, 2001. doi: 10.1021/ac0155411. URL https://doi.org/10.1021/ac0155411. PMID: 11791550.

GORDON I. OGILVIE and MICHAEL R. E. PROCTOR. On the relation between viscoelastic and magnetohydrodynamic flows and their instabilities. Journal of Fluid Mechanics, 476:389-409, 2 2003. ISSN 1469-7645. doi: 10.1017/S0022112002003051. URL http://journals . cambridge.org/article a $_{S} 022112002003051$.

J. M. Ottino. The kinematics of mixing: Stretching, chaos, and transport. Cambridge and New York, Cambridge University Press, 1989, 375 p., 1989.

Giovanni Paladin and Angelo Vulpiani. Anomalous scaling laws in multifractal objects. Physics Reports, 156(4):147 - 225, 1987. ISSN 0370-1573. doi: https://doi.org/10.1016/0370-1573(87)90110-4. URL http://www.sciencedirect.com/science/article/pii/0370157387901104.

Maurizio Quadrio and Paolo Luchini. Integral space-time scales in turbulent wall flows. Physics of Fluids, 15(8):2219-2227, 2003. doi: 10.1063/1.1586273. URL https://doi.org/10.1063/1.1586273.

Markus Raffel, Christian E. Willert, Steven T. Wereley, and Jürgen Kompenhans. Particle Image Velocimetry: A Practical Guide (Experimental Fluid Mechanics). Springer; 2nd edition, September 2007. ISBN 3540723072 .

F. Scarano and M. L. Rhiethmuller. Advances in iterative multigrid piv image processing. Exp. Fluids, 29, 2001.

Charles M. Schroeder, Hazen P. Babcock, Eric S. G. Shaqfeh, and Steven Chu. Observation of polymer conformation hysteresis in extensional flow. Science, 301(5639):1515-1519, 2003. ISSN 0036-8075. doi: 10.1126/science.1086070. URL http://science.sciencemag. org/content/301/5639/1515. 
E S G Shaqfeh. Purely elastic instabilities in viscometric flows. Annual Review of Fluid Mechanics, 28(1):129185, 1996. doi: 10.1146/annurev.fl.28.010196.001021. URL http://dx.doi.org/10.1146/annurev.fl.28.010196.001021.

BI Shraiman and ED Siggia. Scalar turbulence. NATURE, 405(6787):639 646, JUN 8 2000. ISSN 0028-0836. doi: 10.1038/35015000.

Boris I. Shraiman and Eric D. Siggia. Lagrangian path integrals and fluctuations in random flow. Phys. Rev. E, 49: 2912-2927, Apr 1994. doi: 10.1103/PhysRevE.49.2912. URL https://link.aps.org/doi/10.1103/PhysRevE.49.2912.

Boris I. Shraiman and Eric D. Siggia. Scalar turbulence. Nature, 405:639 EP -, 06 2000. URL https://doi.org/10.1038/35015000.

Polly J. Shrewsbury, Susan J. Muller, and Dorian Liepmann. Effect of flow on complex biological macromolecules in microfluidic devices. Biomedical Microdevices, 3(3):225-238, Sep 2001. ISSN 1572-8781. doi: 10.1023/A:1011415414667. URL https ://doi.org/10.1023/A:1011415414667.

Douglas E. Smith, Thomas T. Perkins, and Steven Chu. Dynamical scaling of dna diffusion coefficients. Macromolecules, 29(4):1372-1373, 1996. doi: 10.1021/ma951455p. URL https://doi.org/10.1021/ma951455p.

D. T. Son. Turbulent decay of a passive scalar in the batchelor limit: Exact results from a quantum-mechanical approach. Phys. Rev. E, 59:R3811-R3814, Apr 1999. doi: 10.1103/PhysRevE.59.R3811. URL https://link.aps.org/doi/10.1103/PhysRevE.59.R3811.

Victor Steinberg. Elastic stresses in random flow of a dilute polymer solution and the turbulent drag reduction problem. Comptes Rendus Physique, 10(8):728 - 739, 2009. ISSN 16310705. doi: http://dx.doi.org/10.1016/j.crhy.2009.10.015. URL http://www.sciencedirect.com/science/article/pii/S1631070509001765.

JASON R. STOKES, LACHLAN J. W. GRAHAM, NICK J. LAWSON, and DAVID V. BOGER. Swirling flow of viscoelastic fluids. part 2. elastic effects. Journal of Fluid Mechanics, 429:117-153, 2001a. doi: $10.1017 /$ S0022112000002901.

JASON R. STOKES, LACHLAN J. W. GRAHAM, NICK J. LAWSON, and DAVID V. BOGER. Swirling flow of viscoelastic fluids. part 1 . interaction between inertia and elasticity. Journal of Fluid Mechanics, 429:67-115, 2001b. doi: 10.1017/S0022112000002883.

Abraham D. Stroock, Stephan K. W. Dertinger, Armand Ajdari, Igor Mezić, Howard A. Stone, and George M. Whitesides. Chaotic mixer for microchannels. Science, 295(5555):647651, 2002. ISSN 0036-8075. doi: 10.1126/science.1066238. URL http://science.sciencemag. org/content/295/5555/647. 
R. Sureshkumar, Antony N. Beris, and Marios Avgousti. Nonaxisymmetric subcritical bifurcations in viscoelastic taylor-couette flow. Proceedings of the Royal Society of London A: Mathematical, Physical and Engineering Sciences, 447(1929):135-153, 1994. ISSN 0962-8444. doi: 10.1098/rspa.1994.0132. URL http://rspa.royalsocietypublishing.org/content/447/1929/135.

Shuichi Takayama, Emanuele Ostuni, Philip LeDuc, Keiji Naruse, Donald E. Ingber, and George M. Whitesides. Subcellular positioning of small molecules. Nature, 411:1016 EP -, 06 2001. URL https : //doi.org/10.1038/35082637.

R. I. Tanner. A theory of die-swell. Journal of Polymer Science Part A-2: Polymer Physics, 8(12):20672078, 1970. doi: 10.1002/pol.1970.160081203. URL https://onlinelibrary.wiley.com/doi/abs/10.1002/pol.1970.160081203.

G. I. Taylor. The spectrum of turbulence. Proceedings of the Royal Society of London. Series A - Mathematical and Physical Sciences, 164(919): 476-490, 1938. doi: 10.1098/rspa.1938.0032.

Daniel Therriault, Scott R. White, and Jennifer A. Lewis. Chaotic mixing in three-dimensional microvascular networks fabricated by directwrite assembly. Nature Materials, 2:265 EP -, 03 2003. URL https://doi.org/10.1038/nmat863.

V. Tirtaatmadja and T. Sridhar. A filament stretching device for measurement of extensional viscosity. Journal of Rheology, 37(6):1081-1102, 1993. doi: 10.1122/1.550372. URL https://doi.org/10.1122/1.550372.

B. A. Toms. Proceedings of the international conference on rheology (holland 1949). pages II.135-II.141. North Holland, Amsterdam, 1949.

E. Torricelli. Opera geometrica. 1644.

V Toussaint, P Carriere, and F Raynal. A numerical Eulerian approach to mixing by chaotic advaection. Physics of fluids, 7(11):2587-2600, NOV 1995. ISSN 1070-6631. doi: 10.1063/1.868707.

V Toussaint, P Carriere, J Scott, and JN Gence. Spectral decay of a passive scalar in chaotic mixing. PHYSICS OF FLUIDS, 12(11):2834-2844, NOV 2000. ISSN 1070-6631. doi: 10.1063/1.1290277.

Boubou Traore, Cathy Castelain, and Teodor Burghelea. Efficient heat transfer in a regime of elastic turbulence. Journal of NonNewtonian Fluid Mechanics, 223:62 - 76, 2015. ISSN 03770257. doi: http://dx.doi.org/10.1016/j.jnnfm.2015.05.005. URL http://www.sciencedirect.com/science/article/pii/S0377025715000981.

Jr-Hung Tsai and Liwei Lin. Active microfluidic mixer and gas bubble filter driven by thermal bubble micropump. Sensors 
and Actuators A: Physical, 97-98:665 - 671, 2002. ISSN 09244247. doi: https://doi.org/10.1016/S0924-4247(02)00031-6. URL http://www.sciencedirect.com/science/article/pii/S0924424702000316. Selected papers from Eurosenors XV.

Ravi A. Vijayendran, Kathleen M. Motsegood, David J. Beebe, and Deborah E. Leckband. Evaluation of a three-dimensional micromixer in a surface-based biosensor. Langmuir, 19(5):1824-1828, 2003. doi: 10.1021/la0262250. URL https://doi.org/10.1021/la0262250.

G. V. Vinogradov and V. N. Manin. An experimental study of elastic turbulence. Kolloid-Zeitschrift und Zeitschrift für Polymere, 201(2): 93-98, Feb 1965. ISSN 1435-1536. doi: 10.1007/BF01520490. URL https://doi.org/10.1007/BF01520490.

P. S. Virk and D. L. Wagger. Aspects of mechanisms in type b drag reduction. In Albert Gyr, editor, Structure of Turbulence and Drag Reduction, pages 201-213, Berlin, Heidelberg, 1990. Springer Berlin Heidelberg. ISBN 978-3-642-50971-1.

Z. Warhaft. Passive scalars in turbulent flows. Annual Review of Fluid Mechanics, 32(1):203-240, 2000. doi: 10.1146/annurev.fluid.32.1.203. URL http://dx.doi.org/10.1146/annurev.fluid.32.1.203.

K. Weissenberg. A continuum theory of rhelogical phenomena. Nature, 159: $310 \mathrm{EP}$-, 03 1947. URL https://doi.org/10.1038/159310a0.

George M. Whitesides and Abraham D. Stroock. Flexible methods for microfluidics. Physics Today, 54(6):42-48, 2001. doi: 10.1063/1.1387591. URL https://doi .org/10.1063/1.1387591.

A.L Yarin. On the mechanism of turbulent drag reduction in dilute polymer solutions: dynamics of vortex filaments. Journal of Non-Newtonian Fluid Mechanics, 69(2):137 - 153, 1997. ISSN 0377-0257. doi: https://doi.org/10.1016/S0377-0257(96)01526-1. URL http://www.sciencedirect. com/science/article/pii/S0377025796015261.

K. B. M. Q. Zaman and A. K. M. F. Hussain. Taylor hypothesis and large-scale coherent structures. Journal of Fluid Mechanics, 112:379-396, 1981. doi: 10.1017/S0022112081000463.

J Zilz, R J Poole, M A Alves, D Bartolo, B Levaché, and A Lindner. Geometric scaling of a purely elastic flow instability in serpentine channels. J. Fluid Mech., 712:203-218, October 2012. 